\title{
RENORMALIZATION IN THE HÉNON FAMILY, II: THE HETEROCLINIC WEB
}

\author{
M. LYUBICH, M. MARTENS
}

Abstract. We study highly dissipative Hénon maps

$$
F_{c, b}:(x, y) \mapsto\left(c-x^{2}-b y, x\right)
$$

with zero entropy. They form a region $\Pi$ in the parameter plane bounded on the left by the curve $W$ of infinitely renormalizable maps. We prove that Morse-Smale maps are dense in $\Pi$, but there exist infinitely many different topological types of such maps (even away from $W$ ). We also prove that in the infinitely renormalizable case, the average Jacobian $b_{F}$ on the attracting Cantor set $\mathcal{O}_{F}$ is a topological invariant. These results come from the analysis of the heteroclinic web of the saddle periodic points based on the renormalization theory. Along these lines, we show that the unstable manifolds of the periodic points form a lamination outside $\mathcal{O}_{F}$ if and only if there are no heteroclinic tangencies.

\section{Contents}

1. Introduction

2. Preliminaries

3. Local stable manifolds

4. Laminar structure of the attractor 16

5. Conjugations 28

6. Heteroclinic tangencies $\quad 32$

7. Location of the tip 33

8. The average Jacobian as topological invariant 40

9. The stable lamination 44

10. Morse-Smale components 49

Appendix: Variation of the $\beta_{0}$-unstable manifold 62

Appendix: Open Problems $\quad 65$

Nomenclature $\quad 66$

References $\quad 67$

Date: October 29, 2018. 


\section{INTRODUCTION}

The renormalization theory for the Hénon family began with the works of Collet, Eckman and Koch and Gambaudo, van Strien and Tresser [CEK], GST]. In this paper we continue our exploration of renormalization of Hénon maps started in [CLM]. As Part I was mostly concerned with geometric properties of the Cantor attractor $\mathcal{O}_{F}$, here we focus on global topological properties of the maps in question that are essentially determined by the structure of the web of the stable/unstable manifolds of saddle periodic points (we call it the "heteroclinic web"). As in Part I, we have encountered here some surprising phenomena.

In the one-dimensional situation, all infinitely renormalizable maps with the same combinatorics are topologically equivalent. It is not anymore the case in the Hénon family; in fact, all infinitely renormalizable Hénon maps near the Feigenbaum point are topologically distinct. More generally, the average Jacobian $b_{F}$ of a Hénon-like map is a topological invariant: varying $b_{F}$ leads to bifurcations in the heteroclinic web $(\S 9)$.

Along these lines, we carry out a detailed analysis of the heteroclinic web. In particular, we show that the unstable manifolds form a lamination (outside the attractor $\mathcal{O}_{F}$ ) if and only if there are no heteroclinic tangencies $(\S \S 4,6)$. We also show that the orbit of the "tip" of $\mathcal{O}_{F}$ (a counterpart of the critical value of one-dimensional maps) is topologically distinguished: it is respected by topological conjugacies $(\S 5)$.

Infinitely renormalizable maps in question separate the regions with regular (zero entropy) and chaotic (positive entropy) dynamics, GST] . Because of the Newhouse phenomenon, hyperbolic maps are not dense in the chaotic region. However, it is conceivable that they are dense in the regular region. We confirm this conjecture in a narrow strip to the left of the curve of infinitely renormalizable maps: Morse-Smale maps are dense over there $(\$ 10)$. However, the situation is quite intricate, as there are infinitely many different types of Morse-Smale maps in this region. In particular, a boundary arc of a Morse-Smale component which is not related to the longest periodic orbit is accumulated by infinitely many different Morse-Smale components.

Remark 1.1. The maps in the region we consider, a narrow strip to the left of the curve of infinitely renormalizable maps, do not have homoclinic intersections. Previous results in [PS] and [C] imply that maps in this region can be $C^{1}$ approximated by Morse-Smale maps. 


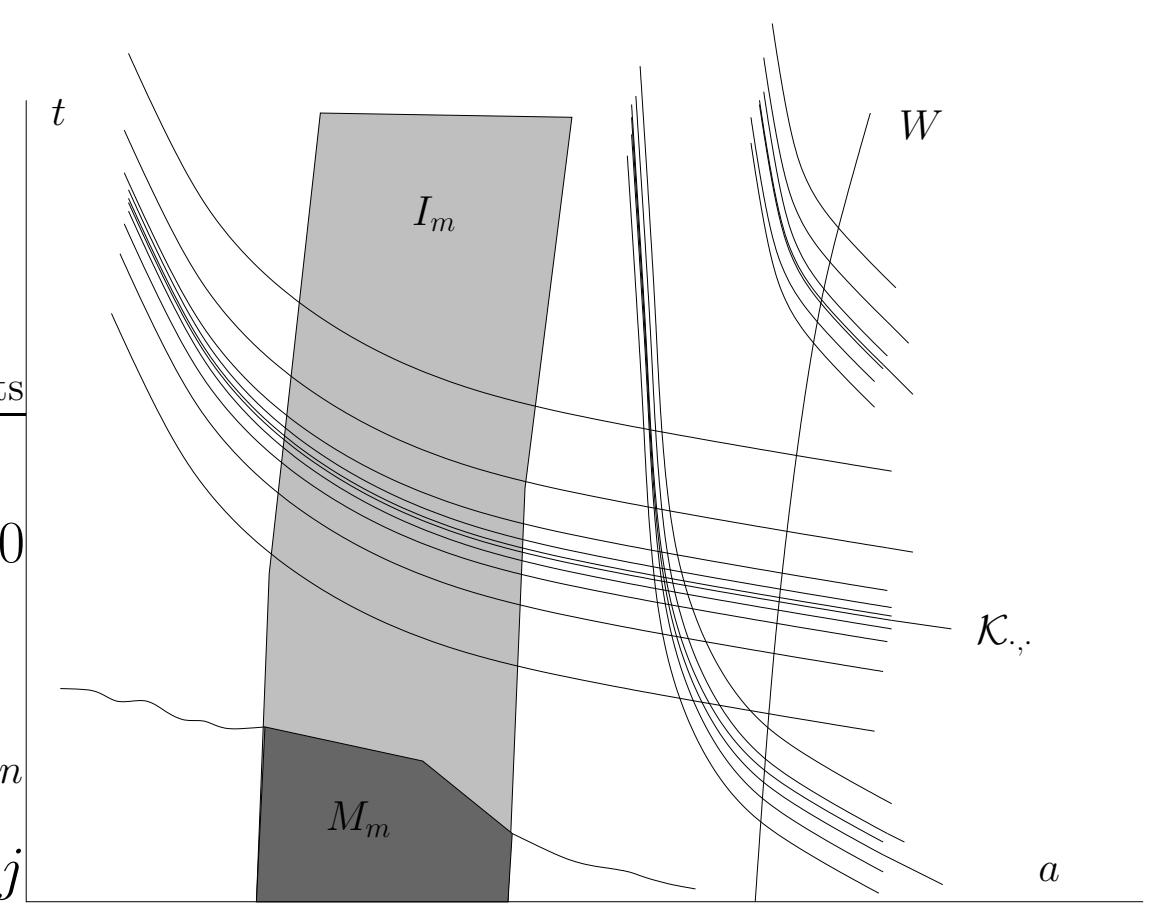

FiguRE 1.1. Bifurcation pattern

The results of $\S 10$ are illustrated in Figure 1.1. It shows an artist's impression of parts of the bifurcation pattern of a Hénon family in a neighborhood of $W$, the curve of infinitely renormalizable maps. The strip $I_{m}$ consists of the $m$-times renormalizable maps. The bottom Morse-Smale component $M_{m}$ is attached to the $m$-times renormalizable unimodal maps. The curves $\mathcal{K}_{\text {., }}$ illustrate loci of heteroclinic tangencies.

\section{Acknowledgements}

We thank Andre de Carvalho and Charles Tresser for many inspiring Hénon discussions. We also thank all the institutions and foundations that have supported us in the course of this work: Simons Mathematics and Physics Endowment, Fields Institute, NSF, NSERC, University of Toronto.

\section{Preliminaries}

The precise definitions and proofs of the following statements can be found in part I, see [CLM], of this series on renormalization of Hénon maps. 
Let $\Omega^{h}, \Omega^{v} \subset \mathbb{C}$ be neighborhoods of $[-1,1] \subset \mathbb{R}$ and $\Omega=\Omega^{h} \times \Omega^{v}$. Let $B=[-1,1] \times[-1,1]$ and $\bar{\epsilon}>0$. The set $\mathcal{H}_{\Omega}(\bar{\epsilon})$ consists of maps $F: B \rightarrow B$ of the following form.

$$
F(x, y)=(f(x)-\epsilon(x, y), x)
$$

where $f:[-1,1] \rightarrow[-1,1]$ is a unimodal map which admits a holomorphic extension to $\Omega^{h}$ and $\epsilon: B \rightarrow \mathbb{R}$ admits a holomorphic extension to $\Omega$ and finally, $|\epsilon| \leq \bar{\epsilon}$. The critical point $c$ of $f$ is non degenerate, $D f(c)<0$. A map in $\mathcal{H}_{\Omega}(\bar{\epsilon})$ is called a Hénon-like map. Observe that Hénon-like maps map vertical lines to horizontal lines.

A unimodal map $f:[-1,1] \rightarrow[-1,1]$ with critical point $c \in[-1,1]$ is renormalizable if $f^{2}:\left[f^{2}(c), f^{4}(c)\right] \rightarrow\left[f^{2}(c), f^{4}(c)\right]$ is unimodal and $\left[f^{2}(c), f^{4}(c)\right] \cap f\left(\left[f^{2}(c), f^{4}(c)\right]\right)=\emptyset$. The renormalization of $f$ is the affine rescaling of $f^{2} \mid\left(\left[f^{2}(c), f^{4}(c)\right]\right.$, denoted by $R f$. The domain of $R f$ is again $[-1,1]$. The renormalization operator $R$ has a unique fixed point $f_{*}:[-1,1] \rightarrow[-1,1]$. The introduction of [FMP presents the history of renormalization of unimodal maps and describes the main results.

The scaling factor of this fixed point $f_{*}$ is

$$
\sigma=\frac{\left|\left[f_{*}^{2}(c), f_{*}^{4}(c)\right]\right|}{|[-1,1]|}
$$

We will also need $\lambda=1 / \sigma=2.6 \ldots$

A Hénon map is renormalizable if there exists a domain $D \subset B$ such that $F^{2}: D \rightarrow D$. The construction of the domain $D$ is inspired by renormalization of unimodal maps. In particular, it is a topological construction. However, for small $\bar{\epsilon}>0$ the actual domain $A \subset B$, used to renormalize as was done in [CLM], has an analytical definition. The precise definition can be found in $\S 3.5$ of part I. If the renormalizable Hénon maps is given by $F(x, y)=(f(x)-\epsilon(x, y))$ then the domain $A \subset B$, an essentially vertical strip, is bounded by two curves of the form

$$
f(x)-\epsilon(x, y)=\text { Const. }
$$

These curves are graphs over the $y$-axis with a slope of the order $\bar{\epsilon}>0$. The domain $A$ satisfies similar combinatorial properties as the domain of renormalization of a unimodal map:

$$
F(A) \cap A=\emptyset,
$$

and

$$
F^{2}(A) \subset A .
$$

Unfortunately, the restriction $F^{2} \mid A$ is not a Hénon-like map as it does not map vertical lines into horizontal lines. This is the reason why the 
coordinated change needed to define the renormalization of $F$ is not an affine map, but it rather has the following form. Let

$$
H(x, y)=(f(x))-\epsilon(x, y), y)
$$

and

$$
G=H \circ F^{2} \circ H^{-1} .
$$

The map $H$ preserves horizontal lines and it is designed in such a way that the map $G$ maps vertical lines into horizontal lines. Moreover, $G$ is well defined on a rectangle $U \times[-1,1]$ of full height. Here $U \subset[-1,1]$ is an interval of length $2 /|s|$ with $s<-1$. Let us rescale the domain of $G$ by the $s$-dilation $\Lambda$, such that the rescaled domain is of the form $[-1,1] \times V$, where $V \subset \mathbb{R}$ is an interval of length $2 /|s|$. Define the renormalization of $F$ by

$$
R F=\Lambda \circ G \circ \Lambda^{-1} .
$$

Notice that $R F$ is well defined on the rectangle $[-1,1] \times V$. The coordinate change $\phi=H^{-1} \circ \Lambda^{-1}$ maps this rectangle onto the topological rectangle $A$ of full height.

The set of $n$-times renormalizable maps is denoted by $\mathcal{H}_{\Omega}^{n}(\bar{\epsilon}) \subset$ $\mathcal{H}_{\Omega}(\bar{\epsilon})$. If $F \in \mathcal{H}_{\Omega}^{n}(\bar{\epsilon})$ we use the notation

$$
F_{n}=R^{n} F \text {. }
$$

The set of infinitely renormalizable maps is denoted by

$$
\mathcal{I}_{\Omega}(\bar{\epsilon})=\bigcap_{n \geq 1} \mathcal{H}_{\Omega}^{n}(\bar{\epsilon})
$$

The collection of maps in $\mathcal{H}_{\Omega}^{n}(\bar{\epsilon})$ which have a periodic attractor of period $2^{n}$ is denoted by $\mathcal{I}_{\Omega}^{n}(\bar{\epsilon})$.

The renormalization operator acting on $\mathcal{H}_{\Omega}^{1}(\bar{\epsilon}), \bar{\epsilon}>0$ small enough, has a unique fixed point $F_{*} \in \mathcal{I}_{\Omega}(\bar{\epsilon})$. It is the degenerate map

$$
F_{*}(x, y)=\left(f_{*}(x), x\right) .
$$

This renormalization fixed point is hyperbolic and the stable manifold has codimension one. Moreover,

$$
W^{s}\left(F_{*}\right)=\mathcal{I}_{\Omega}(\bar{\epsilon}) .
$$

If we want to emphasize that some set, say $A$, is associated with a certain map $F$ we use notation like $A(F)$.

The coordinate change which conjugates $F_{k}^{2} \mid A\left(F_{k}\right)$ to $F_{k+1}$ is denoted by

$$
\phi_{v}^{k}=\left(\Lambda_{k} \circ H_{k}\right)^{-1}: \operatorname{Dom}\left(F_{k+1}\right) \rightarrow A\left(F_{k}\right) .
$$


Here $H_{k}$ is the non-affine part of the coordinate change used to define $R^{k+1} F$ and $\Lambda_{k}$ is the dilation by $s_{k}<-1$. Now, for $k<n$, let

$$
\Phi_{k}^{n}=\phi_{v}^{k} \circ \phi_{v}^{k+1} \circ \cdots \circ \phi_{v}^{n-1}: \operatorname{Dom}\left(F_{n}\right) \rightarrow A_{n-k}\left(F_{k}\right),
$$

where

$$
A_{k}(F)=\Phi_{0}^{k}\left(\operatorname{Dom}\left(F_{k}\right)\right) \cap B .
$$

Notice, that each $A_{k} \subset B$ is of full height and $\Phi_{0}^{k}$ conjugates $R^{k} F$ to $F^{2^{k}} \mid A_{k}$. Furthermore, $A_{k+1} \subset A_{k}$.

Let $n \geq 1$ and $F \in \mathcal{H}_{\Omega}^{n}(\bar{\epsilon})$. The domain of $R^{n} F$ is

$$
\Omega_{n}=\Omega_{n}^{h} \times \Omega_{n}^{v},
$$

where $[-1,1] \subset \Omega_{n}^{h}$. Furthermore, $R^{n} F(x, y)=\left(f_{n}(x)-\epsilon_{n}(x, y), x\right)$.

Lemma 2.1. Given $\Omega$ and $\bar{\epsilon}>0$ small enough, there exist $r>1$ and $C>0$ such that for every $F \in \mathcal{H}_{\Omega}^{n}(\bar{\epsilon})$

$$
\operatorname{diam}\left(\Omega_{n}\right) \leq C \cdot r^{n} .
$$

Proof. Let $k<n$. The maps $f_{k} \mid \Omega_{k}^{h}$ stay within a compact family and $\left|\epsilon_{k}\right|=O\left(\bar{\epsilon}^{2^{k}}\right)$. This is explained in $\S 4$ of [CLM]. Hence, the coordinate changes $\Lambda_{k} \circ H_{k}$ used to define $R^{k+1} F$ as a renormalization of $R^{k} F$ has a uniform bound on its derivative. The Lemma follows.

Remark 2.1. For an infinitely renormalizable map $F \in \mathcal{I}_{\Omega}(\bar{\epsilon})$ the diameters of $\Omega_{n}$ grow exponentially. In particular

$$
\operatorname{diam}\left(\Omega_{n}^{v}\right) \asymp \lambda^{n}
$$

where $\lambda=1 / \sigma=2.6 \ldots$ and $\sigma$ the scaling factor of the unimodal renormalization fixed point. Let $\Phi_{k}^{k+1}: \Omega_{k+1} \rightarrow \Omega_{k}$ be the diffeomorphism which conjugates $R^{k+1} F$ to $\left(R^{k} F\right)^{2} \mid A\left(R^{k} F\right)$. The inverse of this diffeomorphism was constructed in $\S 3.5$ of [CLM]. In fact,

$$
\left(\Phi_{k}^{k+1}\right)^{-1}=\Lambda_{k} \circ H_{k}
$$

where $H_{k}(x, y)=\left(f_{k}(x)-\epsilon_{k}(x, y), y\right)$ and $\Lambda_{k}$ is a dilation. The scaling factor $s_{k}$ of $\Lambda_{k}$ converges exponentially fast: $s_{k} \rightarrow-\lambda$. This is shown in Lemma 7.4 of [CLM]. In particular,

$$
\operatorname{diam}\left(\Omega_{k+1}^{v}\right)=\left|s_{k}\right| \cdot \operatorname{diam}\left(\Omega_{k}^{v}\right) .
$$

Let

$$
B_{v^{n}}=\Phi_{0}^{n}(B)
$$

Notice, for $k<n$

$$
B_{v^{k+1}} \subset B_{v^{k}}
$$


An infinitely renormalizable Hénon-like map has an invariant Cantor set:

$$
\mathcal{O}_{F}=\bigcap_{n \geq 1} \bigcup_{i=0}^{2^{n}-1} F^{i}\left(B_{v^{n}}\right)
$$

Its geometry was discussed in part I. The dynamics on this Cantor set is conjugate to an adding machine. Its unique invariant measure is denoted by $\mu$. The average Jacobian

$$
b_{F}=\exp \int \log \operatorname{Jac} F d \mu
$$

with respect to $\mu$ is an important parameter that essentially influences the geometry of $\mathcal{O}_{F}$, see [CLM].

The critical point (and critical value) of a unimodal map plays a crucial role in its dynamics. The counterpart of the critical value for Hénon-like maps is the tip

$$
\left\{\tau_{F}\right\}=\bigcap_{n \geq 1} B_{v^{n}}
$$

The convergence $R^{n} F \rightarrow F_{*}$ is exponential, $F \in \mathcal{I}_{\Omega}(\bar{\epsilon})$. Theorem 7.9 of [CLM] gives a precise asymptotical form of the convergence. Namely,

$$
R^{n} F(x, y)=\left(f_{n}(x)-b_{F}^{2^{n}} a(x) y\left(1+O\left(\rho^{n}\right)\right), x\right) .
$$

The analytic function $a(x)$ is universal, independent of $F$, and positive, and $\rho<1$. The unimodal part converges exponentially fast: $f_{n} \rightarrow f_{*}$.

We will use the following general notions and notations throughout the text.

A ball in a metric space of radius $r>0$ and centered at $x$ is denoted by $B_{r}(x)$. The diameter of a set is denoted by $\operatorname{diam}(\cdot)$. Let $\pi_{1}$ : $X \times Y \rightarrow X$ and $\pi_{2}: X \times Y \rightarrow Y$ be the projections to resp. the first and second factor.

The graph of a function $\phi: X \rightarrow Y$ is denoted by $\operatorname{graph}(\phi)$. The domain of a map $F$ is denoted by $\operatorname{Dom}(F)$. The image is denoted by $\operatorname{Im}(F)=F(\operatorname{Dom}(F))$.

The tangent space at a point $x \in W$ of a smooth curve $W \subset \mathbb{R}^{2}$ is denoted by $T_{x} W$. If two submanifolds $M_{1}$ and $M_{2}$ (of $M$ ) are tangent at some point we write

$$
M_{1} \Pi M_{2} .
$$

If we want to specify a point of tangency $x \in M_{1} \cap M_{2}$ we write

$$
M_{1} \cap_{x} M_{2} .
$$


The forward orbit of a point or set is denoted by $\operatorname{Orb}(\cdot)=\bigcup_{k \geq 0} f^{k}(\cdot)$. If a point has also a complete backward orbit then the backward and forward orbit together is denoted by $\operatorname{Orb}_{\mathbb{Z}}(\cdot)$. The limit set of a point $x \in \operatorname{Dom}(F)$ is denoted by $\omega(x)$. If a point $x \in \operatorname{Dom}(F)$ has an infinite backward orbit then the limit set of this backward orbit is denoted by $\alpha(x)$. The cycle of a periodic point $\beta, \gamma$ etc. will be called $\boldsymbol{\beta}, \boldsymbol{\gamma}$ etc. The set of periodic points of a map $F$ is denoted by $\mathcal{P}_{F}$. A point $x \in B$ is a wandering point for $F: B \rightarrow B$ if there is a neighborhood $x \in U$ such that $F^{n}(U) \cap U=\emptyset$, for $n \geq 1$. Denote the set of nonwandering points of $F$ by $\Omega_{F}$. Given two points $z, z^{\prime} \in W^{u / s}(x) \subset B$ in the same connected component of $W^{u / s}(x)$, then the arc in $W^{u / s}(x)$ which connects $z$ with $z^{\prime}$ is denoted by $\left[z, z^{\prime}\right]^{u / s}$. When end points of such an arc are deleted we will denoted the remaining arc by $\left(z, z^{\prime}\right]^{u / s}$, $\left(z, z^{\prime}\right)^{u / s}$, etc.

$Q_{1} \asymp Q_{2}$ means that $C^{-1} \leq Q_{1} / Q_{2} \leq C$, where $C>0$ is an absolute constant or depending on, say $F$.

For the reader's convenience, more special notations are collected in the Nomenclature.

\section{LOCAL STABLE MANIFOLDS}

Lemma 3.1. Let $U, U^{\prime}, V^{\prime} \subset \Omega_{h}$ with $\overline{U^{\prime}} \subset V^{\prime}$. Assume, $U^{\prime} \subset \Omega^{v}$. There exists $C>0$ such that the following holds. If $F \in \mathcal{H}_{\Omega}(\bar{\epsilon})$, $F(x, y)=(f(x)-\epsilon(x, y), x)$, and $f: V^{\prime} \rightarrow f\left(V^{\prime}\right)$ is univalent with

$$
f\left(U^{\prime}\right) \supset \bar{U}
$$

then for every $A>0$ there exists $\bar{\epsilon}>0$ such that the following holds. If

$$
\phi: \Omega^{v} \rightarrow U
$$

with

$$
|D \phi| \leq A \cdot \bar{\epsilon}
$$

then the preimage $F^{-1}(\operatorname{graph}(\phi)) \cap\left(U^{\prime} \times \Omega^{v}\right)$ is the graph of some $\psi: \Omega^{v} \rightarrow U^{\prime}$ with

$$
|D \psi| \leq C \cdot \bar{\epsilon}
$$

Remark 3.1. The domains $\Omega^{h}, \Omega^{v}$ are neighborhoods of $[-1,1]$. In the applications of Lemma 3.1 the domains $U$ and $U^{\prime}$ will be small neighborhoods of points in $[-1,1]$. Although formally we have that $U^{\prime} \subset \Omega^{h}$ we can assume in the applications that $U^{\prime} \subset \Omega^{v}$. 
Proof. First we will show that for any given $y \in \Omega^{v}$ there exists a unique $x \in U^{\prime}$ and $y^{\prime} \in \Omega^{v}$ such that

$$
F(x, y)=\left(\phi\left(y^{\prime}\right), y^{\prime}\right) \in \operatorname{graph}(\phi) .
$$

Finding such an $x \in U^{\prime}$ is equivalent to solving

$$
\phi(x)=f(x)-\epsilon(x, y) \equiv \phi_{y}(x) .
$$

This equation is consistent because, $U^{\prime} \subset \Omega^{v}=\operatorname{Dom}(\phi)$. Now, $\phi$ is a strong contraction when $\bar{\epsilon}$ is small, $|D \phi| \leq A \cdot \bar{\epsilon}$. The map $f \mid U^{\prime}$ is univalent. So, for $\bar{\epsilon}$ small enough, the map

$$
\phi_{y}: U^{\prime} \rightarrow \phi_{y}\left(U^{\prime}\right) \supset \bar{U}
$$

is univalent and

$$
\phi_{y}^{-1} \circ \phi: U^{\prime} \rightarrow U^{\prime}
$$

is a well defined contraction. We used again that $U^{\prime} \subset \Omega^{v}=\operatorname{Dom}(\phi)$. The unique fixed point of this map is the point $x \in U^{\prime}$ which solves the equation (3.1). We proved that the set $F^{-1}(\operatorname{graph}(\phi)) \cap\left(U^{\prime} \times \Omega^{v}\right)$ is the graph of some $\psi: \Omega^{v} \rightarrow U^{\prime}$.

Left is to estimate the derivative of $\psi$. Differentiate $\phi(x)=f(x)-$ $\epsilon(x, y)$ with respect to $y$. This gives the following expression

$$
D \psi(y)=-\frac{\frac{\partial \epsilon}{\partial y}(x, y)}{D \phi(x)-D f(x)+\frac{\partial \epsilon}{\partial x}(x, y)} .
$$

There is a lower bound on $|D f(x)| \geq D>0, x \in U^{\prime}$. Furthermore, the partial derivatives of $\epsilon$ are of the order $\bar{\epsilon}$. So, for $\bar{\epsilon}$ small enough, we get

$$
|D \psi(y)| \leq C \cdot \bar{\epsilon}
$$

In the sequel of this section we will fix a $F \in \mathcal{H}_{\Omega}^{n}(\bar{\epsilon}), n \geq 1$. The domain of $R^{n} F$ is

$$
\Omega_{n}=\Omega_{n}^{h} \times \Omega_{n}^{v},
$$

where $[-1,1] \subset \Omega_{n}^{h}$. Furthermore, $R^{n} F(x, y)=\left(f_{n}(x)-\epsilon_{n}(x, y), x\right)$.

Let $\hat{\beta}_{n} \in \Omega_{n-1}$ be the saddle point of $R^{n-1} F$ which is of flip type, it has two negative eigenvalues. The connected component of its stable manifold which contains $\hat{\beta}_{n}$ is denoted by $W_{\text {loc }}^{s}\left(\hat{\beta}_{n}\right)$. This set is called the local stable manifold of $\hat{\beta}_{n}$.

The point $\hat{\beta}_{n} \in \Omega_{n}$ corresponds to a periodic point of the original map. Namely, $\beta_{n}=\Phi_{0}^{n}\left(\hat{\beta_{n}}\right) \in \Omega_{0}$. Objects with a hat are in the domain of a renormalization. The corresponding object in the domain of the original map will have no hat. 
Lemma 3.2. For $\bar{\epsilon}>0$ small enough, the local stable manifold of the point $\hat{\beta}_{n}$ is the graph of a function $\hat{\psi}_{n}: \Omega_{n-1}^{v} \rightarrow \Omega_{n-1}^{h}$ with

$$
\left|D \hat{\psi}_{n}\right|=O\left(\bar{\epsilon}^{2^{n-1}}\right) \text {. }
$$

Proof. The map $f_{n-1}$ lies in a compact family which is determined by $\Omega$. This implies that for some $\delta>0$ and $D>0$ we have the following. Let $U_{n}^{\prime}=U_{n}=B_{\delta}\left(\pi_{1}\left(\hat{\beta}_{n}\right)\right)$ and $V_{n}=B_{2 \delta}\left(\pi_{1}\left(\hat{\beta}_{n}\right)\right)$. Then

$$
\left|D f_{n-1}(x)\right| \geq D>1
$$

for $x \in U_{n}^{\prime}$.

Consider the family of graphs of the following functions:

$$
\mathcal{G}_{K}=\left\{\phi: \Omega_{n-1}^{v} \rightarrow \Omega_{n-1}^{h}\left|\phi\left(\pi_{2}\left(\hat{\beta}_{n}\right)\right)=\pi_{1}\left(\hat{\beta}_{n}\right),\right| D \phi \mid \leq K \cdot \bar{\epsilon}^{2^{n-1}}\right\} .
$$

Notice, for $\phi \in \mathcal{G}_{K}$ we have

$$
\phi\left(\Omega_{n-1}^{v}\right) \subset U_{n}
$$

This follows from Lemma 2.1. Namely,

$$
\operatorname{diam}\left(\phi\left(\Omega_{n-1}^{v}\right)\right) \leq K \cdot \bar{\epsilon}^{2^{n-1}} \cdot \operatorname{diam}\left(\Omega_{n-1}^{v}\right) \leq K \cdot \bar{\epsilon}^{2^{n-1}} \cdot C \cdot r^{n}<\delta .
$$

We can apply Lemma 3.1 which says that, for $\bar{\epsilon}$ small enough, the connected component of $\left(R^{n-1} F\right)^{-1}(\operatorname{graph}(\phi))$ containing $\hat{\beta}_{n}$ is the graph of some function $\psi$. It also says that if we take $K>0$ large enough we have $\psi \in \mathcal{G}_{K}$. This observation defines the graph transform $\mathcal{T}: \mathcal{G}_{K} \rightarrow \mathcal{G}_{K}$ with

$$
\mathcal{T}: \phi \mapsto \psi
$$

The special form of Hénon-like maps allows us to define the graph transform for (global) graphs of $\phi: \Omega_{n-1}^{v} \rightarrow \Omega_{n-1}^{h}$. Because $\left|D f_{n-1}\right| \geq$ $D>1, f_{n-1}$ is expanding, and $\left|\epsilon_{n-1}\right| \leq \bar{\epsilon}^{2^{n-1}}$ we can use the usual technique to show that this graph transform contracts the $C^{0}$ distance on $\mathcal{G}_{K}$. The unique fixed point is $W_{\text {loc }}^{s}\left(\hat{\beta}_{n}\right) \in \mathcal{G}_{K}$. In particular, it is the graph of a function $\hat{\psi}_{n} \in \mathcal{G}_{K}$ :

$$
\left|D \hat{\psi}_{n}\right| \leq K \cdot \bar{\epsilon}^{2^{n-1}} \text {. }
$$

The map $R^{n-1} F$ is renormalizable. It has two fixed points: $\hat{\beta}_{n}=$ $\beta_{1}\left(R^{n-1} F\right)$, which is of flip type, and $\beta_{0}\left(R^{n-1} F\right)$ which has two positive eigenvalues. Let $\hat{p}_{0}^{n} \in W^{u}\left(\beta_{0}\left(R^{n-1} F\right)\right)$ be such that the curve $\left[\beta_{0}\left(R^{n-1} F\right), \hat{p}_{0}^{n}\right] \subset W^{u}\left(\beta_{0}\left(R^{n-1} F\right)\right)$ intersects $W_{\text {loc }}^{s}\left(\hat{\beta}_{n}\right)$ only in $\hat{p}_{0}^{n}$. Let

$$
\hat{p}_{i}^{n}=\left(R^{n-1} F\right)^{i}\left(\hat{p}_{0}^{n}\right), i \in \mathbb{Z} .
$$


The extended local stable manifold of $R^{n-1} F$ consists of four curves contained in $W^{s}\left(\hat{\beta}_{n}\right)$,

$$
\hat{M}^{n}=\left[\hat{p}_{0}^{n}, \hat{\beta}_{n}\right]^{s} \cup\left(R^{n-1} F\right)^{-1}\left(\left[\hat{p}_{0}^{n}, \hat{\beta}_{n}\right]^{s}\right) \cup\left(R^{n-1} F\right)^{-2}\left(\left[\hat{p}_{0}^{n}, \hat{\beta}_{n}\right]^{s}\right),
$$

where $\left[\hat{p}_{0}^{n}, \hat{\beta}_{n}\right]^{s} \subset W^{s}\left(\beta_{n}\right)$ is the curve which connects $\hat{p}_{0}^{n}$ with $\hat{\beta}_{n}$. Lemma 3.1 and Lemma 3.2 imply:

Lemma 3.3. If $\bar{\varepsilon}>0$ is small enough then $\hat{M}^{n} \cap\left(\Omega_{n-1} \cap \mathbb{R}^{2}\right)$ consists of four curves,

(1) $\hat{M}_{-2}^{n} \ni \hat{p}_{-2}^{n}$,

(2) $\hat{M}_{-1}^{n} \ni \hat{p}_{-1}^{n}$,

(3) $\hat{M}_{0}^{n}=W_{\text {loc }}^{s}\left(\hat{\beta}_{n}\right) \ni \hat{\beta}_{n}$,

(4) $\hat{M}_{1}^{n}, W^{u}\left(\beta_{0}\left(R^{n-1} F\right)\right) \cap \hat{M}_{1}^{n}=\emptyset$.

These curves are contained in graphs of functions. These functions, denoted by $\hat{M}_{i}^{n}: \Omega_{n-1}^{v} \rightarrow \Omega_{n-1}^{h}$, have the property that $\operatorname{graph}\left(\hat{M}_{i}^{n}\right) \subset$ $W^{s}\left(\hat{\beta}_{n}\right)$ and

$$
\left|D \hat{M}_{i}^{n}\right|=O\left(\bar{\epsilon}^{2^{n-1}}\right), i=-2,-1,0,1 .
$$

Remark 3.2. For maps $F \in \mathcal{I}_{\Omega}(\bar{\epsilon})$ the bounds on the derivatives in the previous Lemma can be replaced by $O\left(b_{F}^{2^{n-1}}\right)$, where $b_{F}$ is the average Jacobian.

The map $\Phi_{0}^{n}: \Omega_{n} \rightarrow \Omega$ is the coordinate change which conjugates $R^{n} F$ to $F^{2^{n}} \mid A_{n}$, see Equation (2.2). Let

$$
\begin{gathered}
\beta_{n}=\Phi_{0}^{n-1}\left(\hat{\beta}_{n}\right) \in \Omega, \\
p_{i}^{n}=\Phi_{0}^{n-1}\left(\hat{p}_{i}^{n}\right), i \in \mathbb{Z}, \\
M_{i}^{n}=\Phi_{0}^{n-1}\left(\hat{M}_{i}^{n}\right), i=-2,-1,0,1,
\end{gathered}
$$

and

$$
M^{n}=\Phi_{0}^{n-1}\left(\hat{M}^{n}\right) .
$$

Define the domain $D_{1}=D_{1}(F) \subset B$ to be the closed disc bounded by two $\operatorname{arcs} \partial^{s}=\partial^{s}(F) \subset W^{s}\left(\beta_{1}(F)\right)$ and $\partial^{u}=\partial^{u}(F) \subset W^{u}\left(\beta_{0}(F)\right)$ whose boundary points are $p_{0}^{n}$ and $p_{1}^{n}$. Let

$$
D_{n}=\Phi_{0}^{n-1}\left(D_{1}\left(R^{n-1} F\right)\right) .
$$

Notice,

$$
F^{2}\left(D_{1}\right) \subset D_{1}
$$

So

$$
F^{2^{n}}\left(D_{n}\right) \subset D_{n}
$$

The map $F^{2^{n}} \mid D_{n}$ is called the preferred $n^{\text {th }}$-prerenormalization. Finally,

$$
\partial D_{n}=\partial_{n}^{s} \cup \partial_{n}^{u},
$$


where

$$
\partial_{n}^{u, s}=\Phi_{0}^{n-1}\left(\partial^{u, s}\left(R^{n-1} F\right)\right)
$$

Observe,

$$
\left\{\tau_{F}\right\}=\bigcap_{n \geq 0} D_{n},
$$

which holds because $D_{n} \subset B_{v^{n}}$.

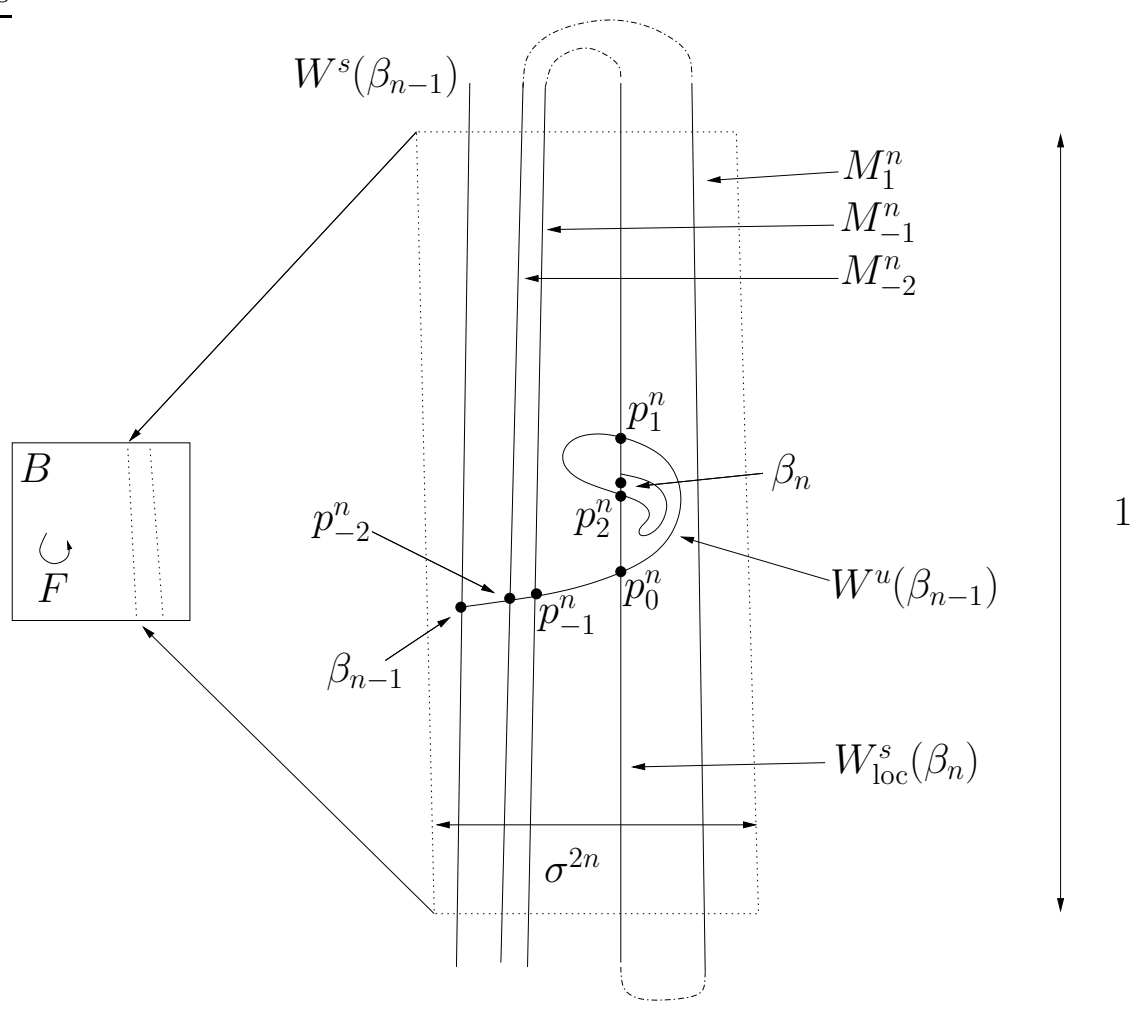

Figure 3.1. Extended local stable manifold

Remark 3.3. The box $A_{n}$, illustrated in Figure 3.1, has unit height and exponential small horizontal width: each horizontal slice is proportional to $\sigma^{2 n}$.

Remark 3.4. The upper index of the points $p_{i}^{n}$ and $\hat{p}_{i}^{n}$ will be omitted when no ambiguity is possible. See for example Figure 4.1 .

Lemma 3.4. For every $C>0$ there exists $K>0$, independent of $N \geq 1$, such that the following holds. Let $\hat{\phi}: \Omega_{n}^{v} \rightarrow \Omega_{n}^{h}$ with

$$
|D \hat{\phi}| \leq C \cdot \bar{\epsilon}^{2^{n}} .
$$


Then $\Phi_{0}^{n}(\operatorname{graph}(\hat{\phi}))$ is the graph of some $\phi: \Omega^{v} \rightarrow \Omega^{h}$ with

$$
|D \phi| \leq K \cdot \bar{\epsilon} .
$$

Proof. In $\S 2$, the preliminaries, we introduced the coordinate change which conjugates $R^{n} F$ with $F^{2^{n}} \mid A_{n}$. Recall that

$$
\Phi_{0}^{n}=\Phi_{0}^{1} \circ \Phi_{1}^{2} \circ \cdots \circ \Phi_{n-1}^{n},
$$

where each $\Phi_{k}^{k+1}$ conjugates $R^{k+1} F$ to the restriction $\left(R^{k} F\right)^{2} \mid A\left(R^{k} F\right)$. These conjugations were constructed in such a way that they map horizontal lines into horizontal lines. Each map

$$
\pi_{2} \circ \Phi_{k}^{k+1}: \Omega_{k+1} \rightarrow \Omega_{k}^{v}
$$

is onto. Moreover, $\pi_{2} \circ \phi_{k}^{k+1}(x, y)$ is independent of $x$ and affine in $y$. This implies that $\Phi_{k}^{n}(\operatorname{graph}(\hat{\phi}))$ is the graph of some $\phi_{k}: \Omega^{v} \rightarrow \Omega^{h}$. In particular, $\Phi_{0}^{n}(\operatorname{graph}(\hat{\phi}))$ is the graph of some $\phi: \Omega^{v} \rightarrow \Omega^{h}$.

Observe that the graph of $\phi_{k}$ stays away from $x=c_{k}, c_{k}$ is the critical point of $f_{k}$. In particular, there exists $D>0$ such that

$$
\left|D f_{k}(x)\right| \geq D
$$

for every $(x, y) \in \operatorname{graph}\left(\phi_{k}\right)$.

The coordinate change $\Phi_{k}^{k+1}$ is a composition of two maps, see the preliminaries in $\S 2$,

$$
\Lambda_{k} \circ H_{k} \equiv\left(\Phi_{k}^{k+1}\right)^{-1}
$$

where

$$
H_{k}(x, y)=\left(f_{k}(x)-\epsilon_{k}(x, y), y\right) .
$$

We will estimate $\left|D \phi_{k}\right|$ inductively. Let $K_{k}>0$ be minimal such that $\left|D \phi_{k}\right| \leq K_{k} \cdot \bar{\epsilon}^{2^{k}}$. In particular, $K_{n} \leq C$. Assume $\left|D \phi_{k+1}\right| \leq K_{k+1} \cdot \bar{\epsilon}^{2^{k+1}}$. Choose a point $(x, y) \in \operatorname{graph}\left(\phi_{k}\right)$ and let $\Phi_{k}^{k+1}\left(x^{\prime}, y^{\prime}\right)=(x, y)$ with $\left(x^{\prime}, y^{\prime}\right) \in \operatorname{graph}\left(\phi_{k+1}\right)$. Take a tangent vector $\left(D \phi_{k}(y) z, z\right)$ to the graph of $\phi_{k}$. Then

$$
D \Lambda_{k} \circ D H_{k}(x, y)\left(D \phi_{k}(y) z, z\right)=\left(D \phi_{k+1}\left(y^{\prime}\right) z^{\prime}, z^{\prime}\right) .
$$

$D \Lambda_{k}$ is conformal which implies that for some $s \in \mathbb{R}$ we have $z^{\prime}=s \cdot z$. Hence,

$$
\left(D f_{k}(x)-\frac{\partial \epsilon_{k}(x, y)}{\partial x}\right) \cdot D \phi_{k}(y)-\frac{\partial \epsilon_{k}(x, y)}{\partial y}=D \phi_{k+1}\left(y^{\prime}\right)
$$

Using $\left|D f_{k}(x)\right| \geq D$ and the above equation we get, for $\bar{\epsilon}$ small enough, constants $A_{0}, A_{1}>0$, independent of $N$, such that

$$
\left|D \phi_{k}\right| \leq A_{0} \cdot\left|D \phi_{k+1}\right|+A_{1} \cdot \bar{\epsilon}^{2^{k}} .
$$


Hence,

$$
K_{k} \leq A_{0} \cdot \bar{\epsilon}^{2^{k}} \cdot K_{k+1}+A_{1} .
$$

This implies that there is a uniform bound $K \geq K_{k}, k \geq 1$.

Proposition 3.5. If $F \in \mathcal{H}_{\Omega}^{n}(\bar{\varepsilon})$, with $\bar{\varepsilon}>0$ small enough, then $M^{n} \cap B$ consists of four curves,

(1) $M_{-2}^{n} \ni p_{-2}^{n}$,

(2) $M_{-1}^{n} \ni p_{-1}^{n}$,

(3) $M_{0}^{n}=W_{\text {loc }}^{s}\left(\beta_{n}\right) \ni \beta_{n}$,

(4) $M_{1}^{n}, M_{1}^{n} \cap W^{u}\left(\beta_{n-1}\right)=\emptyset$.

These curves are contained in graphs of functions. These functions, denoted by $M_{i}^{n}: \Omega^{v} \rightarrow \Omega^{h}$, have the property that $\operatorname{graph}\left(M_{i}^{n}\right) \subset W^{s}\left(\beta_{n}\right)$ and satisfy

$$
\left|D M_{i}^{n}\right|=O(\bar{\epsilon}), \quad i=-2,-1,0,1 .
$$

Letting $z_{n}$ be the intersection point of $M_{1}^{n}$ with the horizontal line through $\tau_{F}$, we have: $\pi_{1}\left(z_{n}\right)>\pi_{1}\left(\tau_{F}\right)$ and

$$
\left|z_{n}-\tau_{F}\right| \asymp \sigma^{2 n}
$$

Proof. The first part of the Proposition follows by applying Lemma 3.3 and Lemma 3.4. The second, which describes the distance from $\tau_{F}$ to $z_{n}$, is an immediate consequence of the results in $\S 7.2$ of [CLM].

Let $W_{n} \subset B$ be the real domain bounded by $M_{0}^{n}$ and $M_{1}^{n}$. The domain $W_{n}$ is a topologically defined variation of $A_{n}$ (the restriction $F^{2^{n}} \mid A_{n}$ is conjugate to the $n^{t h}$-renormalization of $F$, see $\left.\S 2\right)$. Note,

$$
\beta_{n} \in W_{n}
$$

and

$$
\beta_{n}^{\prime} \equiv F^{2^{n-1}}\left(\beta_{n}\right) \in W_{n-1} .
$$

The connected component of the stable manifold $W^{s}\left(\beta_{n}^{\prime}\right) \cap B$ which contains $\beta_{n}^{\prime}$ is called the local stable manifold of $\beta_{n}^{\prime}$, denoted by $W_{\mathrm{loc}}^{s}\left(\beta_{n}^{\prime}\right)$.

Proposition 3.6. For $\bar{\epsilon}>0$ small enough the local stable manifold $W_{\mathrm{loc}}^{s}\left(\beta_{n}^{\prime}\right)$ is the graph of a function $\phi:[-1,1] \rightarrow[-1,1]$ and

$$
|D \phi|=O(\bar{\epsilon}) \text {. }
$$

Proof. The maximal $k \leq n$ for which $R^{k} F$ has a periodic point corresponding to $\beta_{n}^{\prime}$ is $k=n-2$. Namely,

$$
\beta_{n}^{\prime}=\Phi_{0}^{n-2}\left(R^{n-2} F\left(\Phi_{n-2}^{n-1}\left(\hat{\beta}_{n}\right)\right)\right) .
$$

Let

$$
\begin{gathered}
G_{1}=W_{\mathrm{loc}}^{s}\left(\hat{\beta}_{n}\right) \subset \operatorname{Dom}\left(F_{n-1}\right), \\
G_{2}=W_{\mathrm{loc}}^{s}\left(\Phi_{n-2}^{n-1}\left(\hat{\beta}_{n}\right)\right) \subset \operatorname{Dom}\left(F_{n-2}\right),
\end{gathered}
$$


and

$$
G_{3}=W_{\mathrm{loc}}^{s}\left(F_{n-2}\left(\Phi_{n-2}^{n-1}\left(\hat{\beta}_{n}\right)\right)\right) \subset \operatorname{Dom}\left(F_{n-2}\right)
$$

Observe,

$$
W_{\text {loc }}^{s}\left(\hat{\beta}_{n}\right)=\Phi_{0}^{n-2}\left(G_{3}\right) .
$$

Lemma 3.3. (3) says that $G_{1}$ is the graph of a function with small derivative. Then Lemma 3.4 implies that also $G_{2}$ is the graph of a function with small derivative. Use Lemma 3.1 to show that $G_{3}$ is the graph of a function with small derivative. Finally, we get that $W_{\mathrm{loc}}^{s}\left(\beta_{n}^{\prime}\right)$ is the graph of a function with small angle because of Lemma 3.4.

Consider an infinitely renormalizable $F \in \mathcal{I}_{\Omega}(\bar{\epsilon})$, with $\bar{\epsilon}>0$ small enough. Observe, the tip of $F$ satisfies

$$
\tau_{F} \in W_{n},
$$

for all $n \geq 1$. Also, notice that $W_{n} \subset W_{n-1}$. Let

$$
W_{\mathrm{loc}}^{s}\left(\tau_{F}\right)=\bigcap_{n \geq 1} W_{n} .
$$

This set is called the local stable manifold of the tip.

Proposition 3.7. For $\bar{\epsilon}>0$ small enough, the local stable manifold $W_{\mathrm{loc}}^{s}\left(\tau_{F}\right)$ is the graph of an analytic function $\phi:[-1,1] \rightarrow[-1,1]$ and

$$
|D \phi|=O(\bar{\epsilon}) \text {. }
$$

Proof. Let $\phi_{n}: \Omega^{v} \rightarrow \Omega^{h}$ be the function whose graph is $M_{0}^{n}=$ $W_{\text {loc }}^{s}\left(\beta_{n}\right)$. This graph contains the left boundary $M_{0}^{n}$ of $W_{n}$. According to Proposition 3.5 we have a uniform bound on $\phi_{n}: \Omega^{v} \rightarrow \Omega^{h}$. Normality implies $\phi_{n} \rightarrow \phi$. The analyticity of $\phi$ follows. The real slice of the graph of $\phi$ is $W_{\text {loc }}^{s}\left(\tau_{F}\right)$.

The characteristic exponents of the invariant measure on $\mathcal{O}_{F}$ are 0 and $\ln b_{F}$, see Theorem 6.3 of [CLM]. The next Proposition states that the local stable manifold of the tip is indeed part of its stable manifold. However, we do not know the actual value of the stable exponent at the tip.

Proposition 3.8. For $\bar{\epsilon}>0$ small enough,

$$
\operatorname{diam}\left(F^{t}\left(W_{\mathrm{loc}}^{s}\left(\tau_{F}\right)\right)\right)=O\left(\left(\sqrt{b_{F}}\right)^{t}\right),
$$

$t \geq 0$. Also, if $v \in T_{\tau_{F}} W_{\mathrm{loc}}^{s}\left(\tau_{F}\right)$ is a non-zero tangent vector then

$$
\limsup _{t \rightarrow \infty} \frac{1}{t} \ln \left|D F^{t}\left(\tau_{F}\right) v\right| \leq \frac{1}{2} \ln b_{F} .
$$


Proof. Choose $t \geq 0$ and let $n \geq 0$ be maximal such that

$$
2^{n} \leq t .
$$

For $t \geq 0$ large we have a precise description of $R^{n} F$, see Theorem 7.9 of [CLM] or equation (2.3). In particular, by using this result, we get

$$
\operatorname{diam}\left(R^{n} F\left(W_{\mathrm{loc}}^{s}\left(\tau_{R^{n} F}\right)\right)\right)=O\left(b_{F}^{2^{n}} \cdot \operatorname{diam}\left(\Omega_{n}^{v}\right)\right) .
$$

Remark 2.1 gives

$$
\left.\operatorname{diam}\left(\Omega_{n}^{v}\right)\right) \asymp \frac{1}{\sigma^{n}} .
$$

The renormalization microscope described in $\S 5$ of [CLM can be used to construct $F^{t}\left(W_{\mathrm{loc}}^{s}\left(\tau_{F}\right)\right)$. Recall,

$$
\phi_{c}^{k}=\Phi_{k}^{k+1},
$$

and

$$
\phi_{v}^{k}=R^{k} F \circ \Phi_{k}^{k+1},
$$

with $k \geq 0$. The renormalization microscope was made such that there exists a sequence $\omega_{k} \in\{v, c\}, k=1,2, \ldots, n$, such that

$$
F^{t}\left(W_{\mathrm{loc}}^{s}\left(\tau_{F}\right)\right)=\phi_{\omega_{1}}^{1} \circ \phi_{\omega_{2}}^{2} \circ \cdots \circ \phi_{\omega_{n}}^{n}\left(R^{n} F\left(W_{\mathrm{loc}}^{s}\left(\tau_{R^{n} F}\right)\right)\right) .
$$

Lemma 5.1 of [CLM] says that each $\phi_{\omega}^{k}$ is a contraction. In fact,

$$
\left|D\left(\phi_{\omega_{1}}^{1} \circ \phi_{\omega_{2}}^{2} \circ \cdots \circ \phi_{\omega_{n}}^{n}\right)\right| \leq C \sigma^{n} .
$$

Hence,

$$
\operatorname{diam}\left(F^{t}\left(W_{\mathrm{loc}}^{s}\left(\tau_{F}\right)\right)\right)=O\left(\left(\sqrt{b_{F}}\right)^{t}\right),
$$

where we used that $2^{n}>\frac{1}{2} t$. The proof of the infinitesimal version is the same.

\section{LAMinaR STRUCTURE OF THE ATTRACTOR}

For a map $F: B \rightarrow \mathbb{R}^{2}$, the set $\bigcap_{k>0} F^{k}(B)$ is called the global attracting set of $F$. It is the maximal backward invariant subset of $B$. For a discussion on the concept of attractor see [Mi1] and [Mi2].

For an infinitely renormalizable Hénon-like map $F$, let

$$
\mathcal{A}_{F}=\mathcal{O}_{F} \cup \bigcup_{n \geq 0} W^{u}\left(\boldsymbol{\beta}_{n}\right) .
$$

For a map $F \in \mathcal{H}_{\Omega}^{n}(\bar{\varepsilon})$, with $\bar{\varepsilon}>0$ small enough, let $B_{0} \subset B$ be the connected component of $B \backslash W_{\text {loc }}^{s}\left(\beta_{0}\right)$ which contains $\beta_{1}$. The set $\bar{B}_{0}$ consists of non-escaping points. 
Remark 4.1. The set $\mathcal{A}_{F}$ is backward invariant. It is also essentially forward invariant. Notice,

$$
F\left(\mathcal{A}_{F} \cap \bar{B}_{0}\right)=\mathcal{A}_{F} \cap \bar{B}_{0} .
$$

However, $\mathcal{A}_{F} \cap\left(B \backslash \bar{B}_{0}\right)$ is a piece of the unstable manifold of $\beta_{0}$ which is mapped strictly over itself with some points outside of $\mathcal{A}_{F} \cap\left(B \backslash \bar{B}_{0}\right)$.

The following result shows that, in fact, this set $\mathcal{A}_{F}$ is the global attracting set:

Theorem 4.1. Given an infinitely renormalizable Hénon-like map $F \in$ $\mathcal{I}_{\Omega}(\bar{\varepsilon})$ with $\bar{\varepsilon}>0$ small enough, we have:

$$
\mathcal{A}_{F}=\bigcap_{k \geq 0} F^{k}(B)=\overline{W^{u}\left(\beta_{0}\right)}
$$

Furthermore, for every point $x \in B_{0}$ either $x \in W^{s}\left(\boldsymbol{\beta}_{n}\right)$ for some $n \geq 0$ or $\omega(x)=\mathcal{O}_{F}$. The non-wandering set of $F$ is $\Omega_{F}=\mathcal{P}_{F} \cup \mathcal{O}_{F}$.

The second part of Theorem 4.1, concerning the limit sets of points and the non-wandering set, was already proved in [GST]. The proof of this Theorem needs some preparation. For a map $F \in \mathcal{H}_{\Omega}^{n}(\bar{\varepsilon})$, with $\bar{\varepsilon}>0$ small enough, define the $n^{\text {th }}$-trapping region of $F$ as

$$
\operatorname{Trap}_{n}=\operatorname{Orb}\left(D_{n}\right)
$$

Note that

$$
\mathcal{O}_{F} \cup \bigcup_{k \geq n} \boldsymbol{\beta}_{n} \subset \operatorname{int}\left(\operatorname{Trap}_{n}\right)
$$

Lemma 4.2. Let $\bar{\varepsilon}>0$ small enough and $F \in \mathcal{H}_{\Omega}(\bar{\varepsilon})$ be a renormalizable map. For every $x \in B_{0}$, there exists $k \geq 1$ such that

$$
F^{k}(x) \in D_{1} \subset \operatorname{Trap}_{1} .
$$

Let $U \supset \mathcal{A}_{F}$ be a neighborhood. Then there exists $k_{0} \geq 1$ such that for $k \geq k_{0}$

$$
F^{k}\left(\overline{B_{0}}\right) \subset U
$$

Proof. Let $\bar{\varepsilon}>0$ be small enough such that Proposition 3.5 applies. Then we can divide the domain $B_{0}$ by cutting it using the curves $\operatorname{graph}\left(M_{i}^{1}\right), i=-2,-1,0,1$, see Figure 3.1, Let

$$
Z_{1} \cup Z_{2} \cup Z_{3} \cup Z_{4} \cup Z_{5}=B_{0} \backslash \bigcup_{i=-2}^{1} \operatorname{graph}\left(M_{i}^{1}\right),
$$


counting the connected components from left to right. In particular $D_{1} \subset Z_{4}$. The curve in $W^{u}\left(\beta_{0}\right)$ which connects $p_{-1}^{1}$ with $p_{0}^{1}$ is denoted by $\left[p_{-1}^{1}, p_{0}^{1}\right]^{u}$. Let

$$
Z_{3}^{+} \cup Z_{3}^{-}=Z_{3} \backslash\left[p_{-1}^{1}, p_{0}^{1}\right]^{u},
$$

be the partition by $\left[p_{-1}^{1}, p_{0}^{1}\right]^{u} \subset W^{u}\left(\beta_{0}\right)$ of $Z_{3}$ in the connected components. One easily checks the following properties

(1) $F\left(Z_{3}^{+}\right) \subset D_{1}$,

(2) $F\left(Z_{3}^{-}\right) \subset Z_{4}$,

(3) $F\left(Z_{4}\right) \subset Z_{3}^{+}$,

(4) $F\left(Z_{2}\right) \subset Z_{3}$

(5) $F\left(Z_{5}\right) \subset Z_{1} \cup Z_{2}$,

(6) for every $x \in Z_{1}$ there exists $k \geq 1$ such that $F^{k}(x) \in Z_{2}$.

The Lemma follows.

Observe, $\operatorname{Trap}_{1} \cap W^{s}\left(\beta_{0}\right)=\emptyset$. This implies the following.

Corollary 4.3. Let $F \in \mathcal{H}_{\Omega}^{n}(\bar{\varepsilon})$, with $\bar{\varepsilon}>0$ small enough, then there are no homoclinic orbits connected to $\boldsymbol{\beta}_{k}$,

$$
W^{s}\left(\boldsymbol{\beta}_{k}\right) \cap W^{u}\left(\boldsymbol{\beta}_{k}\right)=\emptyset
$$

$k \leq n$.

Let $\Gamma_{j}, j \geq 1$, and $\Gamma$ be smooth curves in the plane. We say that the $\Gamma_{j}$ converge to $\Gamma, \Gamma_{j} \rightarrow \Gamma$, if there are smooth parametrisations of these curves such that the corresponding parametrised curves converge in the $C^{1}$-topology.

Lemma 4.4. Let $F \in \mathcal{H}_{\Omega}^{n}(\bar{\varepsilon})$, with $\bar{\varepsilon}>0$ small enough, and $\Gamma \subset$ $W^{u}\left(\beta_{n}\right)$. Then there are arcs $\Gamma_{j} \subset W^{u}\left(\beta_{0}\right)$ and $t_{j} \rightarrow \infty$ such that $F^{t_{j}}\left(\Gamma_{j}\right) \rightarrow \Gamma$.

Proof. Note first that it is sufficient to prove the assertion for some arc $\Gamma \subset W^{u}\left(\beta_{n}\right)$ containing $\beta_{n}$ in its interior (since $\cup_{k \geq 0} F^{k}(\Gamma)=W^{u}\left(\beta_{n}\right)$ ).

The proof goes by induction. For $n=1$ the Lemma can be proved as follows. As before, let $p_{0}=p_{0}^{1}$ be the first intersection of $W^{u}\left(\beta_{0}\right)$ with $W_{\text {loc }}^{s}\left(\beta_{1}\right)$. The two manifolds intersect transversally. If $\Gamma \subset W^{u}\left(\beta_{1}\right)$ is a curve containing $\beta_{1}$ then the $\lambda$-Lemma (see Chapter 2 Lemma 7.1 of $\mathrm{dMP}$ ) allows us to choose arcs

$$
\Gamma_{1} \supset \Gamma_{2} \supset \Gamma_{3} \supset \cdots \ni\left\{p_{0}\right\}
$$

and times $t_{j} \rightarrow \infty$ such that $F^{t_{j}}\left(\Gamma_{j}\right) \rightarrow \Gamma$.

Assume the Lemma holds for $n-1$. Take an arc $\Gamma \subset W^{u}\left(\beta_{n}\right)$ containing $\beta_{n}$ in its interior, say $\Gamma=\Psi_{0}^{n-1}(\hat{\Gamma})$ with $\hat{\Gamma} \subset W^{u}\left(\beta_{1}\left(R^{n-1} F\right)\right)$. For $\bar{\varepsilon}>0$ small enough, all the renormalizations $R^{k} F, k \leq n-1$, 
belong to the class $\mathcal{H}_{\Omega^{\prime}}(\bar{\varepsilon})$ with some $\Omega^{\prime} \subset \Omega$. In particular, we can apply the base of induction to $\beta_{0}\left(R^{n-1} F\right)$ and $\beta_{1}\left(R^{n-1} F\right)$. This gives a sequence of curves $\hat{\Gamma}_{j} \subset W^{u}\left(\beta_{0}\left(R^{n-1} F\right)\right)$ and $\hat{t}_{j} \rightarrow \infty$ such that

$$
\left(R^{n} F\right)^{\hat{t}_{j}}\left(\hat{\Gamma}_{j}\right) \rightarrow \hat{\Gamma} \subset W^{u}\left(\beta_{1}\left(R^{n} F\right)\right) .
$$

Now, the induction assumption allows us to approximate the curves $\Gamma_{j}=\Psi_{0}^{n}\left(\hat{\Gamma}_{j}\right) \subset W^{u}\left(\beta_{n}\right)$ by curves from $W^{u}\left(\beta_{0}\right)$ and the Lemma follows.

Proof of Theorem 4.1. First we will prove that every point converges to a periodic point or to the Cantor set. Let $x \in B_{0}$ be a point that does not converge to any periodic orbit.

According to Lemma 4.2 there exists $k_{1} \geq 1$ such that

$$
F^{k_{1}}(x) \in D_{1}(F) \text {. }
$$

Notice that

$$
D_{1}(F) \subset B_{v}^{1}(F) \subset \operatorname{Im} \phi_{v}^{1},
$$

where the map $\phi_{v}^{1}$ is defined in (2.1). Now

$$
x_{1}=\left(\phi_{v}^{1}\right)^{-1}\left(F^{k_{1}}(x)\right) \in B_{0}(R F),
$$

because the orbit of $x$ does not converge to the periodic orbit of $\beta_{1}(F)$. Again, using Lemma 4.2, there is $k_{2} \geq 1$ such that

$$
F^{k_{2}}\left(x_{1}\right) \in D_{1}(R F) .
$$

In particular,

$$
\operatorname{Orb}\left(F^{k_{2}}\left(x_{1}\right)\right) \subset \operatorname{Orb}\left(D_{1}(R F)\right)
$$

So,

$$
F^{k_{1}+2 k_{2}}(x) \in D_{2} \subset \operatorname{Trap}_{2} .
$$

Note again that $D_{1}(R F) \subset \operatorname{Im} \phi_{v}^{2}$. Because $R^{n} F \in \mathcal{H}_{\Omega^{\prime}}(\bar{\varepsilon})$, we are allowed to repeatedly apply Lemma 4.2 . Hence for every $n \geq 1$ there exists $k \geq 1$ such that

$$
F^{k}(x) \in \operatorname{Trap}_{n}
$$

Thus

$$
\omega(x)=\mathcal{O}_{F} .
$$

Obviously, $\mathcal{O}_{F} \cup \mathcal{P}_{F} \subset \Omega_{F}$. Take a point $x \in B$ that does not converge to any periodic orbit and is not in the Cantor set $\mathcal{O}_{F}$. The argument above gives for every $n \geq 1$ a neighborhood $U$ of $x$ and $k_{0} \geq 1$ such that for $k \geq k_{0}$

$$
F^{k}(U) \subset \operatorname{Trap}_{n}
$$

For $n \geq 1$ large enough we have $x \notin \operatorname{Trap}_{n}$. Thus, the point is wandering. 
Let us now consider a non-periodic point $x \in W^{s}\left(\boldsymbol{\beta}_{n}\right)$. According to Lemma 4.2 there are disjoint neighborhoods $U$ of $x$ and $V \supset \mathcal{A}_{F}^{n}$, $n \geq 1$, such that for $k \geq k_{0} F^{k}(U) \subset V$. Thus, the point is wandering. This completes the proof of

$$
\Omega_{F}=\mathcal{P}_{F} \cup \mathcal{O}_{F}
$$

Since $\mathcal{A}_{F}$ is backward invariant,

$$
\mathcal{A}_{F} \subset \bigcap_{k \geq 0} F^{k}(B)
$$

The opposite inclusion is obtained as follows. Choose a point $x \in$ $\cap_{k \geq 0} F^{k}(B)$. If $x \in \mathcal{O}_{F}$ we have $x \in \mathcal{A}_{F}$. Assume $x \notin \mathcal{O}_{F}$. For every $j \geq 0$ we have $F^{-j}(x)$ exists. Let $\alpha(x) \subset B$ consists of all limits of the negative orbit of $x$. This is a closed forward and backward invariant set. Choose $n \geq 1$ large enough such that

$$
x \notin \operatorname{Trap}_{n} .
$$

Because, $F\left(\operatorname{Trap}_{n}\right) \subset \operatorname{Trap}_{n}$ we have for every $j \geq 0$

$$
F^{-j}(x) \notin \operatorname{Trap}_{n} .
$$

Observe, $\mathcal{O}_{F} \subset \operatorname{int}\left(\operatorname{Trap}_{n}\right)$. So

$$
\alpha(x) \cap \mathcal{O}_{F}=\emptyset .
$$

Now, the orbit of every point not in $\mathcal{P}_{F}$ converges to $\mathcal{O}_{F}$. Hence,

$$
\alpha(x) \subset \mathcal{P}_{F} .
$$

This in turn implies that $x \in W^{u}\left(\boldsymbol{\beta}_{n}\right)$ for some $n \geq 1$. This completes the proof of

$$
\mathcal{A}_{F}=\bigcap_{k \geq 0} F^{k}(B) .
$$

The closure of the unstable manifold of $\beta_{0}$ is backward invariant. Hence,

$$
\overline{W^{u}\left(\beta_{0}\right)} \subset \bigcap_{k \geq 0} F^{k}(B)=\mathcal{A}_{F} .
$$

The opposite inclusion is obtained as follows. The stable and unstable manifolds are analytic. This implies that there are only countably many heteroclinic points. In particular, there are points in $W^{u}\left(\beta_{0}\right)$ which do not converge to any periodic orbit. These points converge to the Cantor set. Hence,

$$
\mathcal{O}_{F} \subset \overline{W^{u}\left(\beta_{0}\right)}
$$

Lemma 4.4 implies that for every $n \geq 1$

$$
W^{u}\left(\beta_{n}\right) \subset \overline{W^{u}\left(\beta_{0}\right)} \text {. }
$$


Hence,

$$
\mathcal{A}_{F}=\overline{W^{u}\left(\beta_{0}\right)} \text {. }
$$

The proof of the following Lemma is the same as the part of the proof of Theorem 4.1 dealing with the non-wandering set and will be omitted.

Lemma 4.5. Let $F \in \mathcal{I}_{\Omega}^{n}(\bar{\varepsilon})$, with $\bar{\varepsilon}>0$ small enough. Then

$$
\Omega_{F}=\mathcal{P}_{F} .
$$

Definition 4.1. Let $F \in \mathcal{H}_{\Omega}^{n}(\bar{\varepsilon})$, with $\bar{\varepsilon}>0$ small. A point

$$
z \in W^{u}\left(\beta_{k^{\prime}}\right) \subset \bigcup_{k \leq l \leq n} W^{u}\left(\boldsymbol{\beta}_{l}\right)
$$

is laminar if for any sequence $z_{m} \in W^{u}\left(\beta_{k_{m}}\right)$ with $z_{m} \rightarrow z$ and $k \leq$ $k_{m} \leq n$ the following holds

$$
T_{z_{m}} W^{u}\left(\beta_{k_{m}}\right) \rightarrow T_{z} W^{u}\left(\beta_{k}\right) .
$$

The attractor of $F \in \mathcal{H}_{\Omega}^{n}(\bar{\varepsilon})$ is called laminar if every point in

$$
\bigcup_{n \geq 0} W^{u}\left(\boldsymbol{\beta}_{n}\right)=\mathcal{A}(F) \backslash \mathcal{O}(F)
$$

is laminar.

Remark 4.2. If $\mathcal{A}_{F}$ is laminar then every point of $\mathcal{A}_{F} \backslash \mathcal{O}_{F}$ has a neighborhood which is a $C^{1}$-diffeomorphic image of $(-1,1) \times Q$, where $Q \subset[-1,1]$ is a countable set. It has a local product structure. The set $\mathcal{A}_{F} \backslash \mathcal{O}_{F}$ is a match-box manifold, see [AM].

Indeed, it requires work to show that the neighborhood can be linearized by a $C^{1+\alpha}$-coordinate change. It relies on the linearizability of saddle points, see $[\mathrm{H}]$. More on Hölder laminations can be taken from [PSW] and references therein.

The transverse sections can be described as follows. Choose a point $x \in W^{u}\left(\boldsymbol{\beta}_{n}\right) \subset \mathcal{A}_{F} \backslash \mathcal{O}_{F}$ then

$$
Q=\bigcup_{k=0}^{n} Q_{k}
$$

where

(1) $Q_{k} \cap Q_{l}=\emptyset$ when $k \neq l$,

(2) $Q_{k}$ is countable and discrete, $k<n$,

(3) $Q_{n}=\{x\}$ 
(4) for $k<n$

$$
\overline{Q_{k}}=\bigcup_{l \geq k} Q_{l} .
$$

Moreover, $Q_{k}$ accumulates at $Q_{k+1}$ with an asymptotic rate. The rate equals $\mu_{k+1} \in(-1,0)$ which is the stable multiplier of $\beta_{k+1}$. More precisely, for each $q \in Q_{k+1}$ there exists a neighborhood $U \ni q$ in $Q$ such that $U \cap Q_{k}=\left\{q_{i}\right\}_{i \geq 1}$ with

$$
\lim _{i \rightarrow \infty} \frac{q-q_{i}}{\mu_{k+1}^{i}}=C \neq 0
$$

Note that the set $Q_{k}$ accumulates from both sides at $q \in Q_{k+1}$.

Theorem 4.6. The attractor of an infinitely renormalizable Hénon map $F \in \mathcal{I}_{\Omega}(\bar{\varepsilon})$, with $\bar{\varepsilon}>0$ small enough, is laminar if there are no heteroclinic tangencies.

The proof of this Theorem needs some preparation. Let $n \geq 1$ and $q_{1}, q_{2}, q_{3} \in W^{u}\left(\beta_{n-1}\right)$ be the first three intersections, coming from $\beta_{n-1}$ along $W^{u}\left(\beta_{n-1}\right)$, with $W_{\text {loc }}^{s}\left(\beta_{n+1}\right)$, and let $q_{2}^{\prime}, q_{3}^{\prime} \in W^{u}\left(\beta_{n-1}\right)$ be the second and third intersection with $W_{\text {loc }}^{s}\left(\beta_{n+1}^{\prime}\right)$. Now define the saddle-region $T_{n}$ of $\beta_{n}$ the domain containing $\beta_{n}$ which is bounded by the following four arcs, $\left[q_{2}, q_{2}^{\prime}\right]^{u} \subset W^{u}\left(\beta_{n-1}\right),\left[q_{3}, q_{3}^{\prime}\right]^{u} \subset W^{u}\left(\beta_{n-1}\right)$, $\left[q_{2}, q_{3}\right]^{s} \subset W^{s}\left(\beta_{n+1}\right)$ and $\left[q_{2}^{\prime}, q_{3}^{\prime}\right]^{s} \subset W^{s}\left(\beta_{n+1}^{\prime}\right)$, see Figure 4.1.

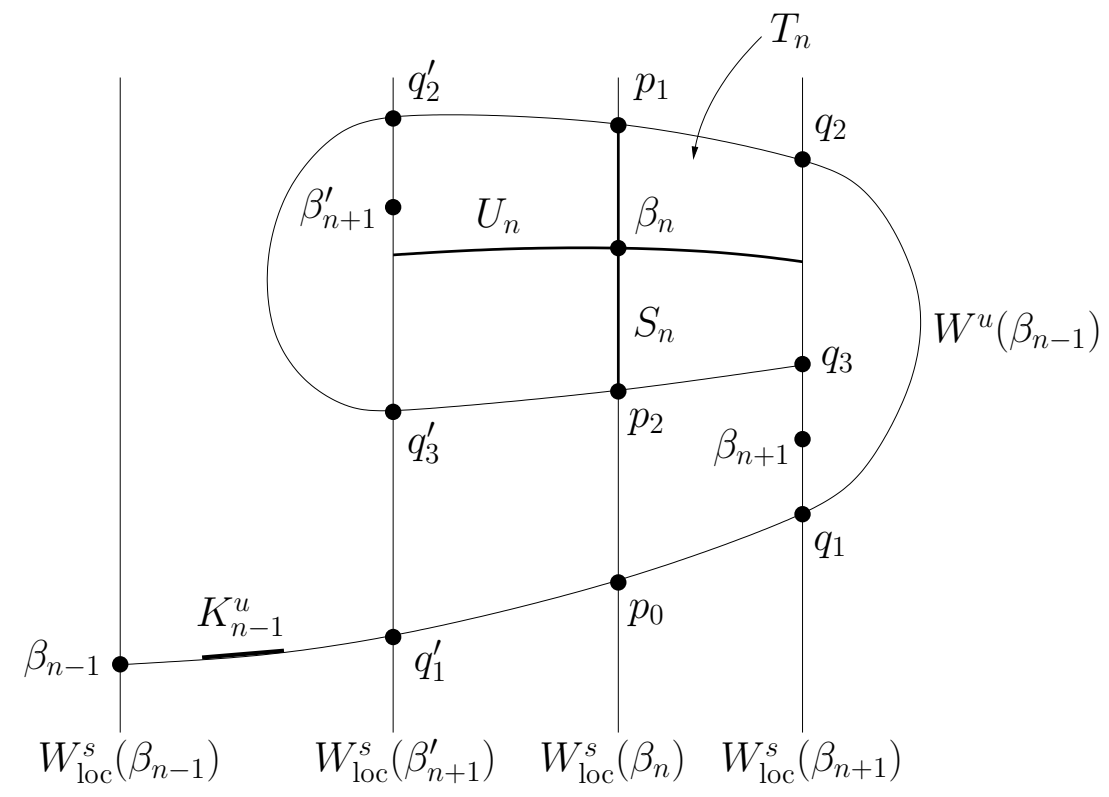

Figure 4.1. Saddle-region of $\beta_{n}$ 
Furthermore, let $p_{0}, p_{1} \in W^{u}\left(\beta_{n-1}\right)$ be the first two intersections with $W_{\text {loc }}^{s}\left(\beta_{n}\right)$. The curve $\left[p_{0}, p_{1}\right]^{u} \subset W^{u}\left(\beta_{n-1}\right)$ is a fundamental domain for $F$ restricted to $W^{u}\left(\beta_{n-1}\right)$. Consider the following fundamental domain

$$
K_{n-1}^{u}=F^{-2^{n}}\left(\left[p_{0}, p_{1}\right]^{u}\right) .
$$

The connected components of $W^{u}\left(\beta_{n}\right) \cap T_{n}$ and $W^{s}\left(\beta_{n}\right) \cap T_{n}$ ( which contain $\left.\beta_{n}\right)$ are denoted by $U_{n}, S_{n} \subset T_{n}$. Notice, $S_{n} \cap \operatorname{Trap}_{n+1}=\emptyset$.

Lemma 4.7. $W^{u}\left(\beta_{n}\right)$ and $W^{s}\left(\beta_{n}\right)$ are (non-compact) one-dimensional embedded manifolds, for each $n \geq 0$.

Proof. For each $n \geq 1$ we have that the fundamental domain of $W^{u}\left(\beta_{n-1}\right)$ satisfies $F^{2^{n}}\left(K_{n-1}^{u}\right) \subset \operatorname{Trap}_{n}$. Because $K_{n-1}^{u} \cap \operatorname{Trap}_{n}=\emptyset$ we have

$$
\overline{\bigcup_{i \neq 0} F^{i}\left(K_{n-1}^{u}\right) \backslash K_{n-1}^{u}} \cap \operatorname{int} K_{n-1}^{u}=\emptyset .
$$

This implies that $W^{u}\left(\beta_{n-1}\right)$ does not accumulate at itself, it is a onedimensional manifold.

The proof for the stable manifold is similar, we have to show that $W^{s}\left(\beta_{n}\right)$ does not accumulate at itself. Suppose it does. Then for some $s \in \operatorname{int} S_{n}$ there exists a sequence $W^{s}\left(\beta_{n}\right) \backslash S_{n} \ni s_{k} \rightarrow s$. We may assume that each $s_{k} \in T_{n}$. Apply Lemma 4.2 to $R^{n} F$ and we get

$$
\beta_{n} \in \omega\left(s_{k}\right) \subset \operatorname{Trap}_{n+1} .
$$

Contradiction.

Remark 4.3. The unstable manifolds are connected. However, the stable manifold of a periodic points consists of countably many closed curves.

Lemma 4.8. Let $F \in \mathcal{H}_{\Omega}^{n}(\bar{\varepsilon})$, with $\bar{\varepsilon}>0$ small enough. Then

$$
W^{s}\left(\beta_{n}\right) \cap T_{n}=S_{n} .
$$

Proof. Let $Z_{3} \subset B$ be the open domain bounded by $M_{-1}^{n}$ and $W_{\text {loc }}^{s}\left(\beta_{n}\right)$ and $Z_{4} \subset B$ be the open domain bounded by $M_{1}^{n}$ and $W_{\text {loc }}^{s}\left(\beta_{n}\right)$, see Figure 3.1. Recall,

$$
F^{2^{n}}\left(Z_{3}\right) \subset Z_{4}
$$

and

$$
F^{2^{n}}\left(Z_{4}\right) \subset Z_{3}
$$

Hence, no point in $Z_{3} \cup Z_{4}$ will ever enter $W_{\text {loc }}^{s}\left(\beta_{n}\right)$. This means

$$
W^{s}\left(\beta_{n}\right) \cap\left(Z_{3} \cup Z_{4}\right)=\emptyset \text {. }
$$

Finally, observe that $T_{n} \subset Z_{3} \cup W_{\text {loc }}^{s} \cup Z_{4}$. The Lemma follows. 
Lemma 4.9. Let $F \in \mathcal{H}_{\Omega}^{n+1}(\bar{\varepsilon})$, with $\bar{\varepsilon}>0$ small enough. Then

$$
\overline{W^{u}\left(\beta_{n}\right)} \backslash W^{u}\left(\beta_{n}\right)=\overline{W^{u}\left(\beta_{n+1}\right)} \cup \overline{W^{u}\left(\beta_{n+1}^{\prime}\right)} .
$$

Proof. Applying Theorem 4.1 to $R F$, we obtain:

$$
\begin{aligned}
\mathcal{A}_{F} \backslash W^{u}\left(\beta_{0}\right) & =\left\{\left(\mathcal{A}_{F} \cap D_{1}\right) \cup\left(\mathcal{A}_{F} \cap F\left(D_{1}\right)\right)\right\} \backslash W^{u}\left(\beta_{0}\right) \\
& \left.=\overline{W^{u}\left(\beta_{1}\right)} \cap D_{1}\right) \cup F\left(\overline{W^{u}\left(\beta_{1}\right)} \cap D_{1}\right) \\
& =\overline{W^{u}\left(\beta_{1}\right)} .
\end{aligned}
$$

Figure 4.1 might be useful in the following argument. Apply Theorem 4.1 and equation (4.2) to $R^{n} F$ and we obtain

$$
\left(\overline{W^{u}\left(\beta_{n}\right)} \backslash W^{u}\left(\beta_{n}\right)\right) \cap D_{n}=\overline{W^{u}\left(\beta_{n+1}\right)} .
$$

Observe that

$$
\overline{W^{u}\left(\beta_{n}\right)} \subset D_{n} \cup F^{2^{n-1}}\left(D_{n}\right) .
$$

Hence,

$$
\begin{aligned}
& \overline{W^{u}\left(\beta_{n}\right)} \backslash W^{u}\left(\beta_{n}\right)= \\
& \left.\left.\left(\overline{\left(W^{u}\left(\beta_{n}\right)\right.} \backslash W^{u}\left(\beta_{n}\right)\right) \cap D_{n}\right) \cup\left(\overline{W^{u}\left(\beta_{n}\right)} \backslash W^{u}\left(\beta_{n}\right)\right) \cap F^{2^{n-1}}\left(D_{n}\right)\right)= \\
& \left.\left(\overline{\left(W^{u}\left(\beta_{n}\right)\right.} \backslash W^{u}\left(\beta_{n}\right)\right) \cap D_{n}\right) \cup F^{2^{n-1}}\left(\overline{\left(W^{u}\left(\beta_{n}\right)\right.} \backslash W^{u}\left(\beta_{n}\right) \cap D_{n}\right)= \\
& \overline{W^{u}\left(\beta_{n+1}\right)} \cup \overline{W^{u}\left(\beta_{n+1}^{\prime}\right)} .
\end{aligned}
$$

Define, for $k<n$,

$$
E_{k, n}=\left\{x \in K_{k} \mid \exists t>0 \forall j<t F^{j}(x) \notin T_{n} \text { and } F^{t}(x) \in T_{n}\right\} .
$$

The time $t>0$ in the above definition is called the time of entry of $x \in E_{k, n}$ into $T_{n}$.

Definition 4.2. Let $k<n$. We say that $F$ satisfies the transversality condition $\mathcal{T}_{k, n}$ if the following holds. Let $z_{j} \in E_{k, n}, j \geq 0$, be a sequence such that

$$
F^{t_{j}}\left(z_{j}\right) \rightarrow s \in S_{n}
$$

where $t_{j}>0$ is the time of entry of $z_{j}$ into $T_{n}$, then

$$
D F^{t_{j}}\left(z_{j}\right)\left(T_{z_{j}} W^{u}\left(\beta_{k}\right)\right) \nrightarrow T_{s} W^{s}\left(\beta_{n}\right) .
$$

Definition 4.3. A $(k, n)$-heteroclinic tangency, $k<n$, for an $n$-times renormalizable Hénon map is a tangency between $W^{u}\left(\beta_{k}\right)$ and $W^{s}\left(\beta_{n}\right)$. If there is such a tangency we write

$$
W^{u}\left(\beta_{k}\right) \pi W^{s}\left(\beta_{n}\right) .
$$


Let $\mathcal{K}_{k, n}(\bar{\varepsilon}) \subset \mathcal{H}_{\Omega}^{n}(\bar{\varepsilon})$ consists of the $n$-times renormalizable maps which have a $(k, n)$-heteroclinic tangency and

$$
\mathcal{U} \mathcal{K}_{k, n}(\bar{\varepsilon})=\bigcup_{k \leq k^{\prime}<n^{\prime} \leq n} \mathcal{K}_{k^{\prime}, n^{\prime}}(\bar{\varepsilon})
$$

Proposition 4.10. Let $F \in \mathcal{H}_{\Omega}^{n}(\bar{\varepsilon}), \bar{\varepsilon}>0$ small enough. Let $k<n$ and suppose that $F$ satisfies

$$
F \notin \mathcal{U} \mathcal{K}_{k, n}(\bar{\varepsilon}) .
$$

Then $\mathcal{T}_{k, n}$ holds.

Proof. Fix $k<n$ and a sequence $z_{j} \in E_{k, n}, j \geq 0$, with $z_{j} \rightarrow z$ and

$$
F^{t_{j}}\left(z_{j}\right) \rightarrow s \in S_{n}
$$

where $t_{j}>0$ in the time of entry of $z_{j}$ into $T_{n}$.

If the $t_{j}$ 's are bounded, say constant $t_{j}=t$, the absence of heteroclinic tangencies, $F \notin \mathcal{K}_{k, n}(\bar{\varepsilon})$, implies that

$$
D F^{t}(z)\left(T_{z} W^{u}\left(\beta_{k}\right)\right) \neq T_{s} S_{n}
$$

Hence,

$$
D F^{t_{j}}\left(z_{j}\right)\left(T_{z_{j}} W^{u}\left(\beta_{k}\right)\right) \nrightarrow T_{s} S_{n}
$$

Secondly, we will consider the case when the times $t_{j}$ of entry are unbounded.

Claim 4.11. There exists $k<m_{1} \leq n$ such that $z \in E_{k, m_{1}} \cap W^{s}\left(\boldsymbol{\beta}_{m_{1}}\right)$.

Proof. Theorem 4.1 describes the limit behavior of the orbit of $z$. Assume,

$$
\omega(z) \subset \mathcal{O}(F) \cup \bigcup_{j>n} \boldsymbol{\beta}_{j} .
$$

Then for some $t>0$ we have $F^{i}(z) \in \operatorname{int}\left(\operatorname{Trap}_{n+1}\right)$ whenever $i \geq t$. This means that the orbit of $z_{j}, j>0$ large enough, will also enter this trapping region after $t$ steps. For $j>0$ large enough, $t_{j}>t$. This contradicts

$$
F^{t_{j}}\left(z_{j}\right) \rightarrow s \notin \operatorname{Trap}_{n+1} .
$$

Denote the time of entry of $z$ into $S_{m_{1}} \subset T_{m_{1}}$ by $r_{1}>0$ and let $F^{r_{1}}(z)=s_{1}$. We will call $r_{1}$ the first transient time. For $j>0$ large enough, $z_{j} \in E_{k, m_{1}}$ with corresponding entry time $t_{j}^{1}=r_{1}$. Note that $m_{1}<n$. Otherwise, the sequence consisting of $t_{j}=t_{j}^{1}=r_{1}$ would be bounded. The absence of heteroclinic tangencies, $F \notin \mathcal{K}_{k, m_{1}}(\bar{\varepsilon})$, implies that

$$
D F^{r_{1}}(z)\left(T_{z} W^{u}\left(\beta_{k}\right)\right) \neq T_{s_{1}} S_{m_{1}}
$$




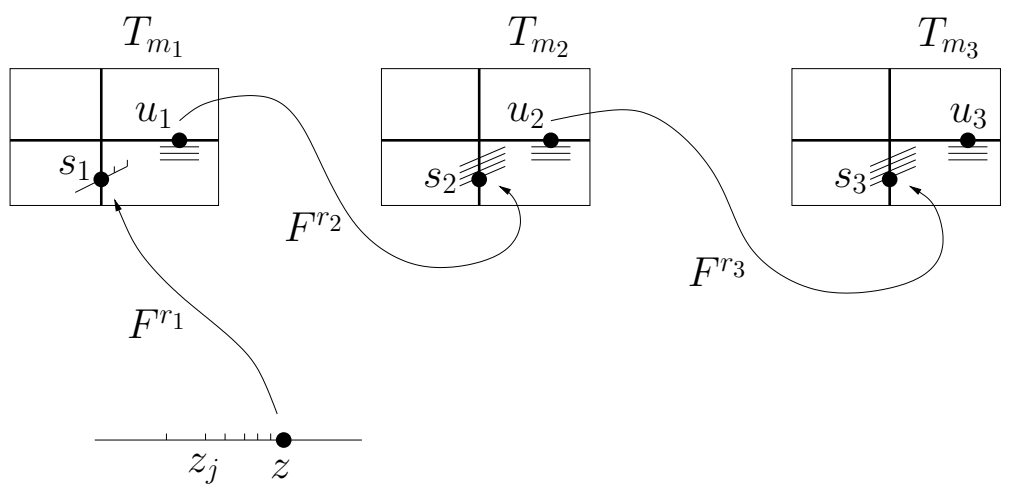

FIGURE 4.2 .

Hence,

$$
D F^{t_{j}^{1}}\left(z_{j}\right)\left(T_{z_{j}} W^{u}\left(\beta_{k}\right)\right) \nrightarrow T_{s_{1}} S_{m_{1}} .
$$

Let $e_{j}^{1}>0$ be maximal such that when $r_{1} \leq i \leq e_{j}^{1}$ we have

$$
F^{i}\left(z_{j}\right) \in T_{m_{1}}
$$

The moment $e_{j}^{1}$ is called the time of exit of $z_{j}$ from $T_{m_{1}}$. We may assume that $F_{j}^{e_{j}^{1}}\left(z_{j}\right) \rightarrow u_{1} \in U_{m_{1}}$. Then (4.3) implies, use the $\lambda$-Lemma from dMP,

$$
D F^{e_{j}^{1}}\left(z_{j}\right)\left(T_{z_{j}} W^{u}\left(\beta_{k}\right)\right) \rightarrow T_{u_{1}} U_{m_{1}} .
$$

Now, we can repeat the proof of Claim 4.11 and obtain $m_{1}<m_{2} \leq n$ and $r_{2}>0$, the second transient time, such that

$$
F^{r_{2}}\left(u_{1}\right)=s_{2} \in S_{m_{2}} .
$$

For $j>0$ large enough we have $z_{j} \in E_{k, m_{2}}$. Denote the time of entry of $z_{j}$ into $T_{m_{2}}$ by $t_{j}^{2}>0$ then $t_{j}^{2}=e_{j}^{1}+r_{2}$. The absence of heteroclinic tangencies, $F \notin \mathcal{K}_{m_{1}, m_{2}}(\bar{\varepsilon})$, implies that

$$
D F^{r_{2}}\left(u_{1}\right)\left(T_{u_{1}} W^{u}\left(\beta_{m_{1}}\right)\right) \neq T_{s_{2}} S_{m_{2}} .
$$

Hence, (4.4) implies

$$
D F^{t_{j}^{2}}\left(z_{j}\right)\left(T_{z_{j}} W^{u}\left(\beta_{k}\right)\right) \nrightarrow T_{s_{2}} S_{m_{2}} .
$$

Let $e_{j}^{2}>0$ be maximal such that when $t_{j}^{2} \leq i \leq e_{j}^{2}$ we have

$$
F^{i}\left(z_{j}\right) \in T_{m_{2}} \text {. }
$$

We may assume that $F^{e_{j}^{2}}\left(z_{j}\right) \rightarrow u_{2} \in U_{m_{2}}$. Then

$$
D F^{e_{j}^{2}}\left(z_{j}\right)\left(T_{z_{j}} W^{u}\left(\beta_{k}\right)\right) \rightarrow T_{u_{2}} U_{m_{2}}
$$


If $m_{2}=n$, statement (4.5) proves the transversality property. In the case when $m_{2}<n$ we can repeat this construction, and we get a sequence $m_{1}<m_{2}<m_{3}<\cdots<m_{g}$ together with points $s_{l} \in S_{m_{l}}$, $u_{l} \in U_{m_{l}}$ and times of entry and exit $t_{j}^{l}>0$ and $e_{j}^{l}>0$ for $z_{j} \in E_{k, m_{l}}$ and the corresponding asymptotic expressions (4.3) and (4.4).

The sequence $m_{l}$ is strictly increasing. Hence, $m_{g}=n$ and $t_{j}=t_{j}^{g}$ for some $g \geq 1$. Now, statement (4.3) corresponding to $T_{m_{g}}$,

$$
D F^{t_{j}}\left(z_{j}\right)\left(T_{z_{j}} W^{u}\left(\beta_{k}\right)\right) \nrightarrow T_{s} S_{n}
$$

finishes the proof of the Proposition.

Proposition 4.12. Let $F \in \mathcal{H}_{\Omega}^{n}(\bar{\varepsilon})$, with $\bar{\varepsilon}>0$ small enough, and $k<n$. Assume

$$
F \notin \mathcal{U} \mathcal{K}_{k+1, n}(\bar{\varepsilon})
$$

Then

$$
\bigcup_{k \leq j \leq n} W^{u}\left(\boldsymbol{\beta}_{j}\right)
$$

is laminar.

Proof. Choose $k \leq j \leq n$. To prove that every point in $W^{u}\left(\beta_{j}\right)$ is laminar it suffices to prove that every point $z \in U_{j}$ is laminar. According to Lemma 4.9 $W^{u}\left(\beta_{j}\right)$ is not accumulated by $W^{u}\left(\beta_{m}\right)$ with $m>j$. From Lemma 4.7 we have that $W^{u}\left(\beta_{j}\right)$ is a one-dimensional embedded manifold. Hence, the only non-trivial accumulation is from $W^{u}\left(\beta_{l}\right)$ with $k \leq l<j$. Assume that $z \in U_{j}$ is not a laminar point. Let $k \leq l<j$ and $z_{m} \in E_{l, j}$ be a sequence with

$$
F^{e_{m}}\left(z_{m}\right) \rightarrow z
$$

but $D F^{e_{m}}\left(z_{m}\right)\left(T_{z_{m}} W^{u}\left(\beta_{l}\right)\right)$ stays away from $T_{z} U_{j}$. Let $t_{m}<e_{m}$ be such that

$$
F^{t_{m}}\left(z_{m}\right) \rightarrow s \in S_{j}
$$

Proposition 4.10 states that $\mathcal{T}_{l, j}$ holds. Hence, for a subsequence, $D F^{t_{m}}\left(z_{m}\right)\left(T_{z_{m}} W^{u}\left(\beta_{l}\right)\right)$ stays away from $T_{s} S_{j}$. Then again the $\lambda$-Lemma implies that for this subsequence

$$
D F^{e_{m}}\left(z_{m}\right)\left(T_{z_{m}} W^{u}\left(\beta_{l}\right)\right) \rightarrow T_{z} U_{j}
$$

Contradiction.

Proof of Theorem 4.6. Suppose, $x \in W^{u}\left(\beta_{n}\right)$ is a non-laminar point of $\mathcal{A}_{F}$. According to Lemma 4.9 this implies that this point is actually a non-laminar point of

$$
\bigcup_{j \leq n} W^{u}\left(\boldsymbol{\beta}_{j}\right) .
$$


This contradicts Proposition 4.12 .

\section{Conjugations}

The boundary $\partial B$ of the domain of a Hénon-like map $F: B \rightarrow B$ does not have dynamical meaning. Even if we restrict the map to $\overline{B_{0}}$, only an arc of $\partial B_{0}$, namely $W_{\text {loc }}^{s}\left(\beta_{0}\right)$, is dynamically meaningful. The fact that the boundary is rather arbitrary entails that the notion of topological equivalence defined by conjugations $h: B \rightarrow h(B)=\tilde{B}$ is too restrictive. For this reason, below we slightly relax this notion.

A relative neighborhood $U \subset \bar{B}_{0}$ is an open set in the intrinsic topology of $B_{0}$. A conjugation between two Hénon-like maps $F, \tilde{F} \in \mathcal{I}_{\Omega}(\bar{\varepsilon})$ is a homeomorphism $h: U \rightarrow h(U)=\tilde{U}$ such that

(1) $U \supset \mathcal{A}_{F}$ and $\tilde{U} \supset \mathcal{A}_{\tilde{F}}$ are relative neighborhoods in the corresponding boxes;

(2) $U$ and $\tilde{U}$ are forward invariant under the corresponding dynamics;

(3) $h \circ F=\tilde{F} \circ h$.

Theorem 5.1. Let $h: U \rightarrow \tilde{U}$ be a conjugation between two infinitely renormalizable Hénon-like maps $F, \tilde{F} \in \mathcal{I}_{\Omega}(\bar{\varepsilon})$, with $\bar{\varepsilon}>0$ small enough. Then

$$
h\left(\operatorname{Orb}_{\mathbb{Z}}\left(\tau_{F}\right)\right)=\operatorname{Orb}_{\mathbb{Z}}\left(\tau_{\tilde{F}}\right) .
$$

The dynamics of $F \mid \mathcal{O}_{F}$, the adding machine, is homogeneous, in the sense that the group of automorphisms acts transitively on $\mathcal{O}_{F}$ (here an automorphism is a homeomorphism commuting with $F$ ). The situation is different when this Cantor set is embedded as the attractor of a Hénon-like map and the automorphism has an extension to a conjugation. Then, as the above Theorem shows, any automorphism has to preserve the orbit of the tip. This easily implies that the automorphism group is reduced to the cyclic group $\mathbb{Z}$ of the iterates of $F \mid \mathcal{O}_{F}$.

The proof of Theorem 5.1 needs some preparation. A map $F \in \mathcal{I}_{\Omega}(\bar{\varepsilon})$ has exactly two fixed points: $\beta_{0}$ and $\beta_{1}$. The first has positive eigenvalues and the second one is of flip type, it has negative eigenvalues. The topological difference between the fixed points imply that every conjugation between two maps satisfies

$$
h\left(\beta_{i}\right)=\tilde{\beta}_{i},
$$

for $i=0,1$. If a Hénon-like map $F$ is renormalizable then there is only one heteroclinic orbit coming from $\beta_{0}$ and going to $\beta_{1}$ :

$$
W^{u}\left(\beta_{0}\right) \cap W^{s}\left(\beta_{1}\right)=\left\{p_{i}^{1}\right\}_{i \in \mathbb{Z}},
$$


with $F\left(p_{i}^{1}\right)=p_{i+1}^{1}$. (The topological definition of renormalizable Hénonlike maps is discussed in $\S 3.4$ of [CLM]). Observe,

$$
p_{0}^{1} \in W^{u}\left(\beta_{0}\right) \subset U=\operatorname{Dom}(h) .
$$

Hence, every conjugation will satisfy

$$
h\left(p_{0}^{1}\right)=\tilde{p}_{m}^{1},
$$

for some $m \in \mathbb{Z}$.

In $\S 3$ the domain $D_{1}$ was introduced, the domain of the first prerenormalization $F^{2} \mid D_{1}$. This topological disc is bounded by two curves $\partial^{s} \subset W^{s}\left(\beta_{1}\right)$ and $\partial^{u} \subset W^{u}\left(\beta_{0}\right)$ whose endpoints are $p_{0}^{1}$ and $p_{1}^{1}$. The forward images of $D_{1}, \partial^{s}$, and $\partial^{u}$ are denoted respectively by $D_{1}^{l}=$ $F^{l}\left(D_{1}\right), \delta_{l}^{s}=F^{l}\left(\partial^{s}\right)$, and $\delta_{l}^{u}=F^{l}\left(\partial^{u}\right), l \geq 0$. The map $F^{l}: D_{1} \rightarrow D_{1}^{l}$ is a diffeomorphism.

For $l \geq 0$, the curve $\delta_{l}^{s} \subset W^{s}\left(\beta_{1}\right)$ connects $p_{l}^{1}$ with $p_{l+1}^{1}$. On the other hand, for $l<0$, and $\bar{\varepsilon}>0$ small enough, there is no arc in $W^{s}\left(\beta_{1}\right)$ which connects $p_{l}^{1}$ with $p_{l+1}^{1}$. The connected components of $W^{s}\left(\beta_{1}\right)$ that contain the points $p_{l}^{1}, l<0$, are pairwise disjoint. This is observed in 93 , see Figure 3.1, and will be useful in what follows.

Lemma 5.2. Let $h: U \rightarrow \tilde{U}$ be a conjugation between $F, \tilde{F} \in \mathcal{I}_{\Omega}(\bar{\varepsilon})$, $\tilde{F} \circ h=h \circ F$. There exists $k, l \geq 0$ and a conjugation

$$
h^{\prime}: V \rightarrow \tilde{V} \text {, }
$$

given by

$$
h^{\prime}=\tilde{F}^{-l} \circ h \circ F^{k}
$$

such that

(1) $D_{1} \subset V, \tilde{D}_{1} \subset \tilde{V}$, and

$$
h^{\prime}\left(D_{1}\right)=\tilde{D}_{1}
$$

(2) for every $x \in V$

$$
h^{\prime}\left(\operatorname{Orb}\left(F^{l}(x)\right)\right)=h\left(\operatorname{Orb}\left(F^{k}(x)\right)\right) \text {. }
$$

Proof. Lemma 4.2 gives a $k \geq 1$ such that $F^{k}\left(\overline{B_{0}}\right) \subset U$. Define a conjugation

$$
h_{1}: B_{0} \rightarrow h_{1}\left(B_{0}\right) \subset \tilde{U} \subset B_{0},
$$

by $h_{1}=h \circ F^{k}$. Observe that the maps $h$ and $h_{1}$ act in the same way on the space of orbits. In particular, equation (5.1) gives some $l \in \mathbb{Z}$ such that

$$
h_{1}\left(p_{0}^{1}\right)=\tilde{p}_{l}^{1} .
$$

The curve $\partial^{s} \subset W^{s}\left(\beta_{1}\right)$ connects $p_{0}^{1}$ with $p_{1}^{1}$. Hence, the points $\tilde{p}_{l}^{1}$ and $\tilde{p}_{l+1}^{1}$ are connected by a curve in the stable manifold of $\tilde{\beta}_{1}$. So, $l \geq 0$. 
The domain of $h_{1}, \operatorname{Dom}\left(h_{1}\right)=B$, contains $D_{1}$. Actually, $h_{1}$ matches the boundaries $\partial^{u, s}$ of $D_{1}$ with the boundaries $\tilde{\delta}^{u, s}$ of $\tilde{D}_{1}^{l}$. Hence,

$$
h_{1}\left(D_{1}\right)=\tilde{D}_{1}^{l} .
$$

As was noticed previously, the map

$$
\tilde{F}^{-l}: \tilde{D}_{1}^{l} \cap \mathcal{A}_{\tilde{F}} \rightarrow \tilde{D}_{1} \cap \mathcal{A}_{\tilde{F}}
$$

is a well-defined homeomorphism because $l \geq 0$. Choose a relatively open $\tilde{F}$-forward invariant set $\tilde{V}^{\prime} \subset B_{0}$ satisfying

$$
\tilde{D}_{1}^{l} \cup \mathcal{A}_{\tilde{F}} \subset \tilde{V}^{\prime} \subset h_{1}\left(B_{0}\right),
$$

and small enough such that $\tilde{F}^{-l} \mid \tilde{V}^{\prime}$ is a well-defined diffeomorphism. Let

$$
\begin{gathered}
V=h_{1}^{-1}\left(\tilde{V}^{\prime}\right), \\
\tilde{V}=\tilde{F}^{-l}\left(\tilde{V}^{\prime}\right),
\end{gathered}
$$

and let $h^{\prime}: V \rightarrow \tilde{V}$ be defined by

$$
h^{\prime}=\tilde{F}^{-l} \circ h_{1}=\tilde{F}^{-l} \circ h \circ F^{k} .
$$

By construction this conjugation satisfies $h^{\prime}\left(D_{1}\right)=\tilde{D}_{1}$.

According to the previous Lemma, we can replace any conjugation by another one which matches the first pre-renormalization domains $D_{1}$ and $\tilde{D}_{1}$, and coincides with the original conjugation on the space of orbits. The following Proposition will complete the proof of Theorem 5.1 .

Proposition 5.3. Let $h: U \rightarrow \tilde{U}$ be a conjugation between two infinitely renormalizable Hénon-like maps $F, \tilde{F} \in \mathcal{I}_{\Omega}(\bar{\varepsilon})$, with $\bar{\varepsilon}>0$ small enough. If $h\left(D_{1}\right)=\tilde{D}_{1}$ then

$$
h\left(D_{n}\right)=\tilde{D}_{n},
$$

for all $n \geq 1$. In particular, $h\left(\tau_{F}\right)=\tau_{\tilde{F}}$.

Proof. First notice that for all $n \geq 0$ we have $D_{n} \subset D_{1} \subset \operatorname{Dom}(h)$ and $\tilde{D}_{n} \subset \tilde{D}_{1} \subset \operatorname{Im}(h)$. So, $h\left(D_{n}\right)$ is well defined.

The proof will be by induction. Assume that $h\left(D_{k}\right)=\tilde{D}_{k}$, for $k \leq n$. There exists a unique periodic point of period $2^{k+1}$ in $D_{k}$. Namely, $\beta_{k+1} \in \operatorname{int}\left(D_{k}\right), k \leq n$. In particular, $h\left(\beta_{k}\right)=\tilde{\beta}_{k}$, with $k \leq n+1$.

Proposition 3.5 gives that both components of $W_{\text {loc }}^{s}\left(\beta_{n+1}\right) \backslash\left\{\beta_{n+1}\right\}$ intersect $\partial_{n}^{u}$. Let $x_{0}, x_{1} \in W_{\text {loc }}^{s}\left(\beta_{n+1}\right) \cap \partial_{n}^{u}$ be the boundary points of the connected component of $W^{s}\left(\beta_{n+1}\right) \cap D_{n}$ containing $\beta_{n+1}$. Say $x_{0}$ is the first and $x_{1}$ is the second intersection of $W^{u}\left(\beta_{n-1}\right)$ with the connected component of $W^{s}\left(\beta_{n+1}\right) \cap D_{n}$ which contains $\beta_{n+1}$. These 


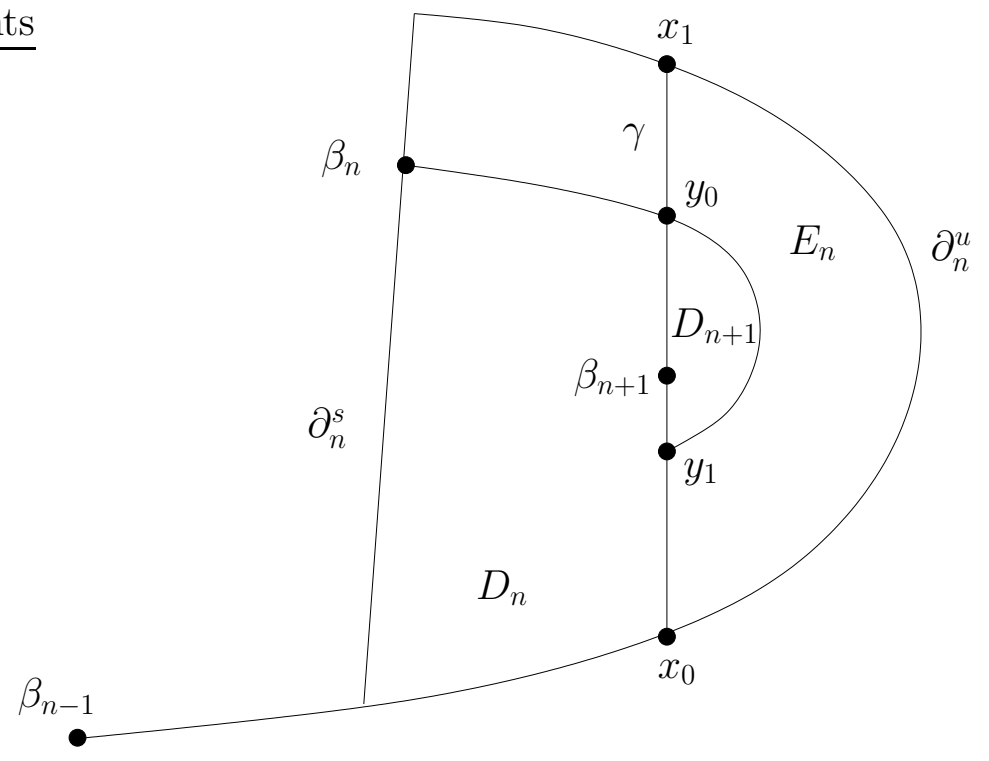

FiguRE 5.1. Domains of pre-renormalizations

points are topologically defined. As was noticed before, $h\left(\beta_{k}\right)=\tilde{\beta}_{k}$, with $k=n-1, n+1$. Hence, for $i=0,1$,

$$
h\left(x_{i}\right)=\tilde{x}_{i} .
$$

Let $\gamma \subset W_{\text {loc }}^{s}\left(\beta_{n+1}\right)$ bounded by $x_{0}$ and $x_{1}$. Then

$$
h(\gamma)=\tilde{\gamma}
$$

Define $E_{n} \subset D_{n}$ to be the connected component of $D_{n} \backslash \gamma$ which does not contain $\beta_{n}$. Then

$$
h\left(E_{n}\right)=\tilde{E}_{n} .
$$

Observe,

$$
D_{n+1} \subset E_{n} .
$$

Let $y_{0}, y_{1} \in W^{u}\left(\beta_{n}\right) \cap \gamma$ be the first and second intersections of $W^{u}\left(\beta_{n}\right)$ and $\gamma$. Then for $i=0,1$

$$
h\left(y_{i}\right)=\tilde{y}_{i} .
$$

Notice, that the arc between $y_{0}$ and $y_{1}$ in $W^{u}\left(\beta_{n}\right)$ equals $\partial_{n+1}^{u}$. Furthermore, the arc between $y_{0}$ and $y_{1}$ in $W^{s}\left(\beta_{n+1}\right)$ equals $\partial_{n+1}^{s}$. Hence, the boundary of $D_{n+1}$ is matched to the boundary of $\tilde{D}_{n+1}$. This finishes the induction step, $h\left(D_{n+1}\right)=\tilde{D}_{n+1}$.

Remark 5.1. Without loss of generality we will only consider conjugations which match the tips of the maps under consideration. 


\section{Heteroclinic tangencies}

If there is a heteroclinic tangency between $W^{u}\left(\beta_{k}\right)$ and $W^{s}\left(\beta_{n}\right)$ then $\beta_{n} \in \mathcal{A}_{F}$ is not a laminar point. Under this circumstances there will be non-periodic points which are non-laminar. Let $\mathcal{C}_{F} \subset \mathcal{A}_{F}$ consists of the non-laminar points. Note, $\mathcal{O}_{F} \subset \mathcal{C}_{F}$.

Any map $F \in \mathcal{H}_{\Omega}^{n}(\bar{\varepsilon})$, with $\bar{\varepsilon}>0$ small enough, has a unique periodic orbit of period $2^{k-1}$, it is the orbit of $\beta_{k}$. Let $\lambda_{k} \in(-1,0]$ and $\mu_{k}<-1$ be the stable and unstable multiplier. The attractor at the $n^{\text {th }}$-scale is

$$
\mathcal{A}_{F}^{n}=\operatorname{Orb}\left(\Psi_{0}^{n}\left(\mathcal{A}_{R^{n} F}\right)\right) \subset \mathcal{A}_{F}, \quad n \geq 0 .
$$

Theorem 6.1. If the infinitely renormalizable Hénon map $F \in \mathcal{I}_{\Omega}(\bar{\varepsilon})$, with $\bar{\varepsilon}>0$ small enough, has an $(k, n)$-heteroclinic tangency and

$$
\frac{\ln \left|\lambda_{k}\right|}{\ln \left|\mu_{n}\right|} \notin \mathbb{Q}
$$

then

$$
\mathcal{A}_{F}^{n} \subset \mathcal{C}_{F}
$$

Proof. We can choose a $C^{1}$-coordinate system for $T_{n}$ such that $U_{n}$ and $S_{n}$ are part of the $x$-axis and $y$-axis resp and that $F^{2^{n}}$ becomes linear with exponents $\lambda_{n}$ and $\mu_{n}$, see $[\mathrm{H}]$. Consider the fundamental domain $\left[1, \mu_{n}^{2}\right]^{u} \subset U_{n}$. Let $z \in S_{n}$ be a $(k, n)$-heteroclinic tangency.

Observe, that there are components of $W_{j} \subset W^{u}\left(\beta_{k-1}\right) \cap T_{k}, j \geq 1$, which accumulate from both sides and in $C^{3}$ sense on $U_{k}$ and they are dynamically related. Namely,

$$
F^{-2^{k}}\left(W_{j+1}\right) \subset W_{j}
$$

This laminar structure of $W^{u}\left(\beta_{k-1}\right)$ around $U_{k}$ will also be visible in a neighborhood of $z \in S_{n}$. Because of the tangency at $z \in S_{n}$ there will be a sequence of points $e_{j} \in W^{u}\left(\beta_{k-1}\right)$ with vertical tangent accumulating at $z \in S_{n}$.

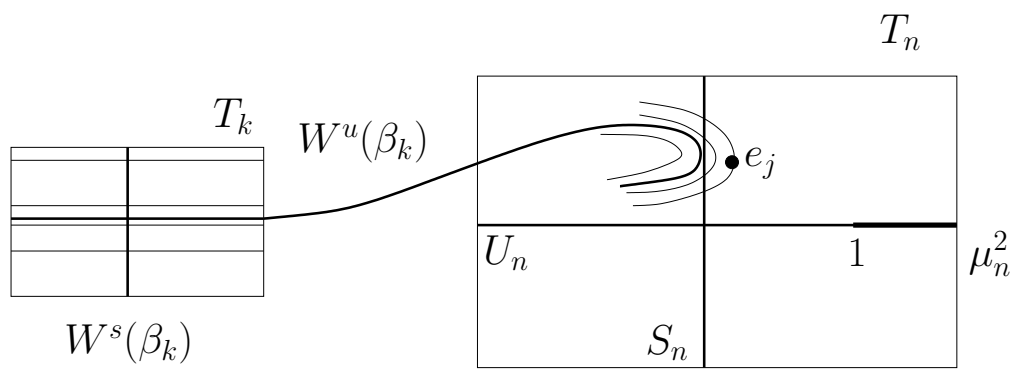

FIGURE 6.1. 
Let $x_{j}$ be the $x$-coordinate of $e_{j}$. Then there is some $\rho<1$ and $C>0$ such that

$$
x_{j}=C \cdot\left(1+O\left(\rho^{j}\right)\right) \cdot \lambda_{k}^{j} .
$$

Notice that accumulation points of $\operatorname{Orb}\left(\left\{e_{j} \mid j>0\right\}\right)$ on $U_{n}$ are nonlaminar points.

For $j>0$ even, let $s_{j}>0$ be the (even) moment when $F^{s_{j}}\left(e_{j}\right)$ is above $\left[1, \mu_{n}^{2}\right]^{u}$,

$$
x_{j} \cdot \mu_{n}^{s_{j}} \in\left[1, \mu_{n}^{2}\right]^{u} .
$$

Let $A=\ln C / \ln \mu_{n}$ then

$$
s_{j}+j \frac{\ln \left|\lambda_{k}\right|}{\ln \left|\mu_{n}\right|}+O\left(\rho^{j}\right)+A=h_{j} \in[0,2] .
$$

Hence,

$$
\frac{h_{j}}{2}=\frac{s_{j}}{2}+\frac{1}{2} \cdot\left(j \cdot \frac{\ln \left|\lambda_{k}\right|}{\ln \left|\mu_{n}\right|}+A\right)+O\left(\rho^{j}\right) \in[0,1] .
$$

Because $s_{j}$ is even we have

$$
h_{j}=2\left\{\frac{1}{2} \cdot j \cdot \frac{\ln \left|\lambda_{k}\right|}{\ln \left|\mu_{n}\right|}+A\right\}+O\left(\rho^{j}\right),
$$

where $\{$.$\} stand for the fractional part. The sequence h_{j}$ is dense in $[0,2]$ because $\frac{\ln \left|\lambda_{k}\right|}{\ln \left|\mu_{n}\right|}$ is irrational. Now, let $\hat{x}_{j}$ be the projection of $F^{s_{j}}\left(e_{j}\right)$ on $U_{n}$. Then

$$
\hat{x}_{j}=\mu_{n}^{h_{j}} .
$$

We proved that $\mathcal{C}_{F}$ contains a fundamental domain of $W^{u}\left(\beta_{n}\right)$. Namely,

$$
\left[1, \mu_{n}^{2}\right]^{u} \subset \mathcal{C}_{F}
$$

The set $\mathcal{C}_{F}$ is closed and invariant. Apply Theorem 4.1 and the proof is finished.

Corollary 6.2. For $\bar{\varepsilon}>0$ small enough, for every $k<n$ there exists a dense $G_{\delta}$ of infinitely renormalizable maps $F \in \mathcal{K}_{k, n}(\bar{\varepsilon}) \cap \mathcal{I}_{\Omega}(\bar{\varepsilon})$ such that

$$
\mathcal{A}_{F}^{n} \subset \mathcal{C}_{F}
$$

\section{LOCATION OF THE TIP}

In this section we will give quantitative information on the location of the tip. Let $F \in \mathcal{I}_{\Omega}(\bar{\epsilon}), \bar{\epsilon}>0$ small enough. The domain bounded by $W_{\text {loc }}^{s}\left(\beta_{2}\right)$ and $W_{\text {loc }}^{s}\left(\beta_{2}^{\prime}\right)$ is called the extended saddle region and denoted by $X \subset B$. 
Consider the following family $\mathcal{W}$ of curves. A curve $\gamma \subset X$ is in $\mathcal{W}$ if it is the graph of a $C^{2}$-function, also denoted by $\gamma$, with

$$
\gamma:\left[x_{\gamma}^{\prime}, x_{\gamma}\right] \rightarrow \mathbb{R}
$$

such that

$$
\left(x_{\gamma}^{\prime}, \gamma\left(x_{\gamma}^{\prime}\right)\right) \in W_{\mathrm{loc}}^{s}\left(\beta_{2}^{\prime}\right)
$$

and

$$
\left(x_{\gamma}, \gamma\left(x_{\gamma}\right)\right) \in W_{\mathrm{loc}}^{s}\left(\beta_{2}\right) .
$$

We will use the notation $\gamma_{\infty}=U_{1} \in \mathcal{W}$, (see Figure 4.1 to recall the definition of $\left.U_{1} \subset B\right)$. The distance $\operatorname{dist}\left(\gamma, \gamma_{\infty}\right)$ is the $C^{2}$-norm between the corresponding functions measured on the domain of $\gamma$. Note that we can always extend $\gamma_{\infty}$ within $W_{\text {loc }}^{u}\left(\beta_{1}\right)$ such that the corresponding domain of this extended function contains the domain $\left[x_{\gamma}^{\prime}, x_{\gamma}\right]$ of any function $\gamma \in \mathcal{W}$.

Let $\Gamma=F(\gamma)$, with $\gamma \in \mathcal{W}$. In particular, $\Gamma_{\infty}=F\left(\gamma_{\infty}\right) \supset \gamma_{\infty}$. Note that each curve $\Gamma$ can be described as a graph over the $y$-axis,

$$
\Gamma:\left[y_{\gamma}^{\prime}, y_{\gamma}\right] \rightarrow \mathbb{R}
$$

This is a consequence of the fact that Hénon-like maps map vertical lines to horizontal lines, $y^{\prime}=x$. Moreover,

$$
\left(\Gamma\left(y_{\gamma}\right), y_{\gamma}\right) \in W_{\mathrm{loc}}^{s}\left(\beta_{2}^{\prime}\right)
$$

and

$$
\left(\Gamma\left(y_{\gamma}^{\prime}\right), y_{\gamma}^{\prime}\right) \in W_{\mathrm{loc}}^{s}\left(\beta_{2}\right) .
$$

Indeed, we will consider the curves $\Gamma=F(\gamma)$ as graphs over the $y$-axis. Note that we can always extend $\Gamma_{\infty}$ within $W_{\text {loc }}^{u}\left(\beta_{1}\right)$ such that the corresponding domain of the extension contains the domain $\left[y_{\gamma}^{\prime}, y_{\gamma}\right]$ of any function $\Gamma$. The distance $\operatorname{dist}\left(\Gamma, \Gamma_{\infty}\right)$ is the $C^{2}$-norm between the corresponding functions measured on the domain of $\Gamma$.

The map $F$ acts on $\mathcal{W}$ as a graph transform. Namely, the curve $W_{\text {loc }}^{s}\left(\beta_{2}\right)$ divide each $\Gamma$ into two components. One of which, denoted by $\mathcal{T}_{F}(\gamma)$, is in $\mathcal{W}$,

$$
\mathcal{T}_{F}: \gamma \mapsto F(\gamma) \cap X
$$

Proposition 7.1. There exists $C>0$, such that for all $F \in \mathcal{I}_{\Omega}(\bar{\epsilon})$, $\bar{\epsilon}>0$ small enough, the following holds:

$$
\operatorname{dist}\left(\Gamma, \Gamma_{\infty}\right) \leq C \cdot b_{F} \cdot \operatorname{dist}\left(\gamma, \gamma_{\infty}\right)
$$

and

$$
\operatorname{dist}\left(\mathcal{T}_{F}(\gamma), \gamma_{\infty}\right) \leq C \cdot b_{F} \cdot \operatorname{dist}\left(\gamma, \gamma_{\infty}\right)
$$




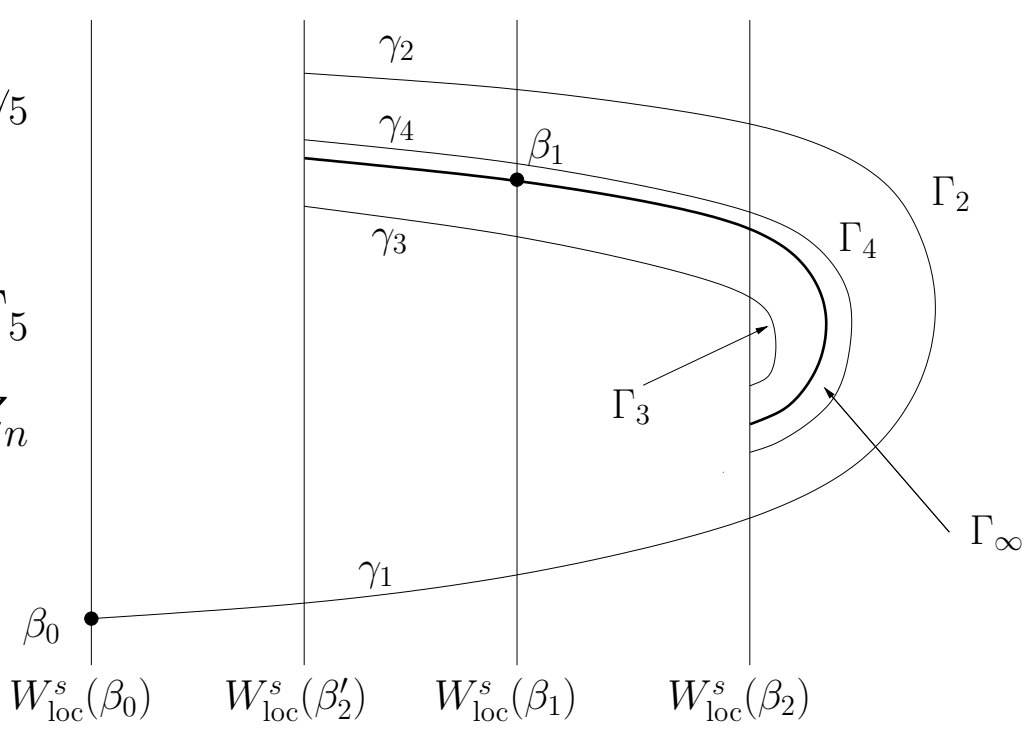

FIGURE 7.1.

If $\left|\gamma(x)-\gamma_{\infty}(x)\right| \in\left[\delta_{1}, \delta_{2}\right]$ with $x \in\left[x_{\gamma}^{\prime}, x_{\gamma}\right]$ then

$$
\left|\mathcal{T}_{F}(\gamma)(x)-\gamma_{\infty}(x)\right| \in\left[\frac{1}{C} \cdot b_{F} \cdot \delta_{1}, C \cdot b_{F} \cdot \delta_{2}\right],
$$

for $x \in\left[x_{\mathcal{T}_{F}(\gamma)}^{\prime}, x_{\mathcal{T}_{F}(\gamma)}\right]$ and

$$
\left|\Gamma(y)-\Gamma_{\infty}(y)\right| \in\left[\frac{1}{C} \cdot b_{F} \cdot \delta_{1}, C \cdot b_{F} \cdot \delta_{2}\right],
$$

for $y \in\left[y_{\gamma}^{\prime}, y_{\gamma}\right]$.

Proof. Let $F \in \mathcal{I}_{\Omega}(\bar{\epsilon})$, say $F(x, y)=(f(x)-\epsilon(x, y), x)$. Inspired by Theorem 7.9 from [CLM], we use the following representation

$$
\epsilon(x, y)=b_{F} a(x) y(1+m(x, y)) .
$$

The correction term $m$ is uniformly bounded for $F \in \mathcal{I}_{\Omega}(\bar{\epsilon})$. The specific form of a Hénon-like map, $F(x, y)=(f(x)-\epsilon(x, y), x)$, implies

$$
\begin{aligned}
\Gamma(y)-\Gamma_{\infty}(y)= & -\epsilon(y, \gamma(y))-\epsilon\left(y, \gamma_{\infty}(y)\right) \\
= & -b_{F} a(y) \cdot\left(\gamma(y)-\gamma_{\infty}(y)\right)+ \\
& -b_{F} a(y) \cdot\left(\gamma(y)-\gamma_{\infty}(y)\right) \cdot m(y, \gamma(y))+ \\
& -b_{F} a(y) \cdot \gamma_{\infty}(y) \cdot\left(m(y, \gamma(y))-m\left(y, \gamma_{\infty}(y)\right) .\right.
\end{aligned}
$$

The uniform bounds on $m$ and its derivatives give immediately the Properties (7.1) and (7.4).

Let $\gamma_{0}: x \mapsto 0$. The expression $F(x, y)=(f(x)-\epsilon(x, y), x)$ implies that $\Gamma_{0}$ is the graph of the unimodal map $f$. Let $Y \subset \operatorname{Dom}\left(\Gamma_{0}\right)$ be such 
that the graph of $\Gamma_{0} \mid Y \subset X$, the extended saddle region. The graph of $f$ has its maximum outside the extended saddle region. This implies that

$$
\left|D \Gamma_{0}\right| Y \mid \geq \delta>0
$$

Also

$$
\left|D \Gamma_{\infty}\right| Y \mid \geq \frac{1}{2} \delta>0
$$

which holds because of (7.1):

$$
\operatorname{dist}\left(\Gamma_{0}, \Gamma_{\infty}\right)=O\left(b_{F}\right)
$$

Now, use in the above expression for the difference of $\Gamma(y)$ and $\Gamma_{\infty}(y)$ the fact that the derivative of $\Gamma_{\infty} \mid Y$ is away from zero: Properties (7.2) and (7.3) follow.

Remark 7.1. According to Theorem 7.9 from [CLM] the correction term $m_{n}$ of $R^{n} F$ decays exponentially.

Let $q_{1}^{\prime}, q_{1} \in W^{u}\left(\beta_{0}\right)$ be the the first intersection, coming from $\beta_{0}$ along $W^{u}\left(\beta_{0}\right)$, with $W_{\text {loc }}^{s}\left(\beta_{2}^{\prime}\right)$ and $W_{\text {loc }}^{s}\left(\beta_{2}\right)$, compare Figure 4.1. Consider the corresponding curve $\gamma_{1}=\left[q_{1}^{\prime}, q_{1}\right]^{u} \subset W^{u}\left(\beta_{0}\right)$. Note, $\gamma_{1} \in \mathcal{W}$. Let

The curves

$$
\gamma_{i}=\mathcal{T}_{F}^{i-1}\left(\gamma_{1}\right), i=2,3, \ldots
$$

$$
\Gamma_{i}=F\left(\gamma_{i-1}\right) \supset \gamma_{i}, i=2,3, \ldots
$$

will be used to locate the tip.

Corollary 7.2. There exists $C>0$, independent of the particular $F \in$ $\mathcal{I}_{\Omega}(\bar{\epsilon})$, such that for $i \geq 2$

$$
\operatorname{dist}\left(\Gamma_{i}, \Gamma_{\infty}\right) \leq C^{i} \cdot b_{F}^{i-1}
$$

and

$$
\frac{1}{C^{i}} \cdot b_{F}^{i-1} \leq\left|\Gamma_{i}(y)-\Gamma_{\infty}(y)\right| \leq C^{i} \cdot b_{F}^{i-1},
$$

for $y \in \operatorname{Dom}\left(\Gamma_{i}\right)$.

Lemma 7.3. There exist unique points $v_{i} \in \Gamma_{i}, i \geq 2$, and $v_{\infty} \in \Gamma_{\infty}$ with a vertical tangent direction. Moreover,

$$
\frac{d \Gamma_{i}}{d y} \asymp-\left(y-v_{i}\right), i=2,3, \ldots, \infty .
$$

Proof. Let $F(x, y)=(f(x)-\epsilon(x, y), x)$ and $\gamma_{0}: x \mapsto 0$. Then $\Gamma_{0}=$ $F\left(\gamma_{0}\right)$ is the graph of $f$ over the $y$-axis. Proposition 7.1 and Corollary 7.2 imply that in $C^{2}$ sense we have

$$
\operatorname{dist}\left(\Gamma_{0}, \Gamma_{i}\right) \leq \operatorname{dist}\left(\Gamma_{0}, \Gamma_{\infty}\right)+\operatorname{dist}\left(\Gamma_{\infty}, \Gamma_{i}\right) \leq C \cdot b_{F}+C^{i} \cdot b_{F}^{i-1}<<1,
$$


for $i=2,3 \ldots$ The Lemma follows because $f$ has a non degenerate critical point.

Let $a$ be the intersection point of the horizontal line through $\tau=\tau_{F}$ and $\Gamma_{2}$. Note, $a$ is to the right of $\tau$. In case we are analyzing these points of the renormalization $R^{k} F$, we will use the notation $v_{i}^{k} \in \Gamma_{i}^{k}$, $i=2,3, \ldots, \infty$, the points with vertical tangency.

Proposition 7.4. For all $F \in \mathcal{I}_{\Omega}(\bar{\epsilon}), \bar{\epsilon}>0$ small enough, the following holds.

(1) $\left|\pi_{1}\left(v_{2}\right)-\pi_{1}(a)\right|=O\left(b_{F}^{2}\right)$,

(2) $\left|\pi_{2}\left(v_{2}\right)-\pi_{2}(a)\right|=O\left(b_{F}\right)$,

(3) $\left|v_{2}-\tau\right|=O\left(b_{F}\right)$,

(4) $|\tau-a| \asymp b_{F}$.

Remark 7.2. The proof of Proposition 7.4 is illustrated with Figures 7.2 and 7.3. The $x$-direction has been stretched dramatically. The analysis is done in a small neighborhood of $v_{\infty}^{k+1}$ in Figure 7.2 and around $v_{\infty}$ in Figure 7.3. So, in the actual picture the curves are essentially straight vertical lines and the difference between the $x$-coordinates $\hat{x}_{k+1}, x_{k+1}$, and $x_{k+1}^{\prime}$ would be invisible.

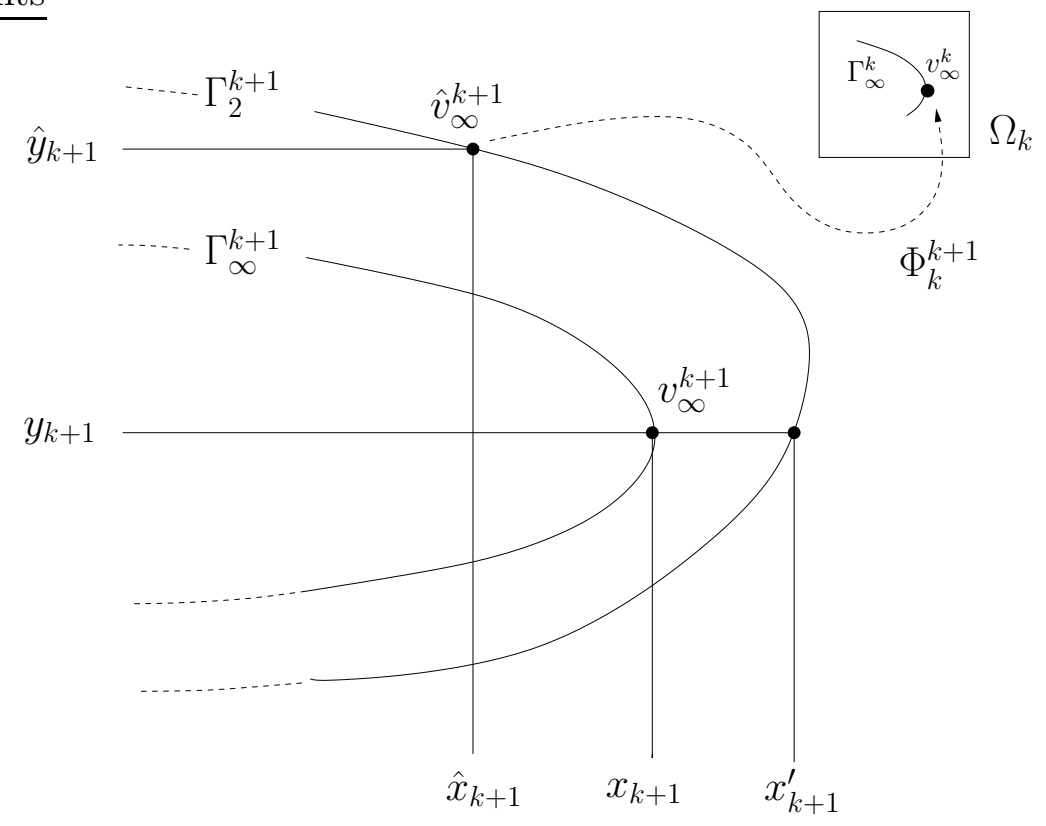

FIGURE 7.2 . 
Proof. Take $k \geq 0$ and consider $R^{k+1} F$. Observe,

$$
v_{\infty}^{k} \in \Phi_{k}^{k+1}\left(\Gamma_{2}^{k+1}\right) \subset \Phi_{k}^{k+1}\left(W^{u}\left(\beta_{0}\left(R^{k+1} F\right)\right)\right) .
$$

So, we can choose a point $\hat{v}_{\infty}^{k+1} \in \Gamma_{2}^{k+1} \subset \Omega_{k+1}$ such that

$$
\Phi_{k}^{k+1}\left(\hat{v}_{\infty}^{k+1}\right)=v_{\infty}^{k} \in D_{2}\left(R^{k} F\right) .
$$

In coordinates, see Figure 7.2 ,

$$
\hat{v}_{\infty}^{k+1}=\left(\hat{x}_{k+1}, \hat{y}_{k+1}\right)
$$

and

$$
v_{\infty}^{k+1}=\left(x_{k+1}, y_{k+1}\right)
$$

Remember,

$$
\Lambda_{k} \circ H_{k} \equiv\left(\Phi_{k}^{k+1}\right)^{-1}
$$

with

$$
H_{k}(x, y)=\left(f_{k}(x)-\epsilon_{k}(x, y), y\right) .
$$

The affine map $\Lambda_{k}$ is a dilatation. Consider $D H_{k}$ and observe that this derivative maps the vertical tangent vector at $v_{\infty}^{k}$ to $\Gamma_{\infty}^{k}$ to an almost vertical vector. The angle between the image vector and the vertical is of the order $b_{F}^{2^{k}}$. Then, because $\hat{v}_{\infty}^{k+1}=\Lambda_{k} \circ H_{k}\left(v_{\infty}^{k}\right)$ and $D \Gamma_{\infty}^{k}\left(y_{k}\right)=0$, we get

$$
\left|D \Gamma_{2}^{k+1}\left(\hat{y}_{k+1}\right)\right|=O\left(b_{F}^{2^{k}}\right) .
$$

Let

$$
x_{k+1}^{\prime}=\pi_{1}\left(\Gamma_{2}^{k+1}\left(y_{k+1}\right)\right) .
$$

Corollary 7.2 gives

$$
\left|x_{k+1}-x_{k+1}^{\prime}\right| \asymp b_{F}^{2^{k+1}}
$$

and

$$
\left|D \Gamma_{2}^{k+1}\left(y_{k+1}\right)\right|=O\left(b_{F}^{2^{k+1}}\right) .
$$

The estimates (7.5) and (7.7) together with Lemma 7.3 gives,

$$
\left|\hat{y}_{k+1}-y_{k+1}\right|=O\left(b_{F}^{2^{k}}\right) \text {. }
$$

The derivative of $\Gamma_{2}^{k+1}$ is bounded, see Lemma 7.3. Use this and the estimates (7.6) and (7.8) to get

$$
\begin{aligned}
\left|\hat{x}_{k+1}-x_{k+1}\right| & \leq\left|\hat{x}_{k+1}-x_{k+1}^{\prime}\right|+\left|x_{k+1}^{\prime}-x_{k+1}\right| \\
& \leq\left|\hat{y}_{k+1}-y_{k+1}\right| \cdot\left|D \Gamma_{2}^{k+1}\right|+O\left(b_{F}^{2^{k+1}}\right)=O\left(b_{F}^{2^{k}}\right) .
\end{aligned}
$$

This and estimate (7.8) implies

$$
\left|\hat{v}_{\infty}^{k+1}-v_{\infty}^{k+1}\right|=O\left(b_{F}^{2^{k}}\right) .
$$


The map $\Phi_{k}^{k+1}$ has a uniformly bounded derivative. So

$$
\left|v_{\infty}^{k}-\Phi_{k}^{k+1}\left(v_{\infty}^{k+1}\right)\right|=O\left(b_{F}^{2^{k}}\right) .
$$

Lemma 5.1 of [CLM] gives, for $k \geq 0$,

$$
\left|\Phi_{0}^{k}\left(v_{\infty}^{k}\right)-\Phi_{0}^{k+1}\left(v_{\infty}^{k+1}\right)\right|=O\left(\sigma^{k} \cdot b_{F}^{2^{k}}\right) .
$$

Notice,

$$
\Phi_{0}^{k}\left(v_{\infty}^{k}\right) \in D_{k}
$$

This implies

$$
\Phi_{0}^{k}\left(v_{\infty}^{k}\right) \rightarrow \tau
$$

for $k \rightarrow \infty$. The estimates (17.9) implies

$$
\left|v_{\infty}-\tau\right| \leq \sum_{k=0}^{\infty}\left|\Phi_{0}^{k}\left(v_{\infty}^{k}\right)-\Phi_{0}^{k+1}\left(v_{\infty}^{k+1}\right)\right|=O\left(b_{F}\right) .
$$

Introduce the following notation, see Figure 7.3 ,

Corollary 7.2 gives

$$
\begin{gathered}
\tau=(x, y), \\
a=\left(x_{a}, y\right), \\
v_{\infty}=\left(u_{\infty}, w_{\infty}\right), \\
\Gamma_{2}\left(w_{\infty}\right)=u_{2}^{\prime}, \\
v_{2}=\left(u_{2}, w_{2}\right) .
\end{gathered}
$$

$$
\left|u_{\infty}-u_{2}^{\prime}\right| \asymp b_{F},
$$

and

$$
\mid D \Gamma_{2}\left(w_{\infty}\right)=O\left(b_{F}\right) .
$$

By definition,

$$
\mid D \Gamma_{2}\left(w_{2}\right)=0
$$

So, Lemma 7.3 gives

$$
\left|w_{\infty}-w_{2}\right|=O\left(b_{F}\right) .
$$

The second derivative of $\Gamma_{2}$ is bounded, which implies

$$
\left|u_{2}-u_{2}^{\prime}\right|=O\left(\left|w_{\infty}-w_{2}\right|^{2}\right)=O\left(b_{F}^{2}\right) .
$$

The estimates in (17.10) and (7.12) imply

$$
\left|y-w_{2}\right| \leq\left|y-w_{\infty}\right|+\left|w_{\infty}-w_{2}\right|=O\left(b_{F}\right) .
$$

We proved (2). Use estimate (7.14) and Lemma 7.3 to get

$$
\left|x_{a}-u_{2}\right|=O\left(\left|y-w_{2}\right|^{2}\right)=O\left(b_{F}^{2}\right) \text {. }
$$




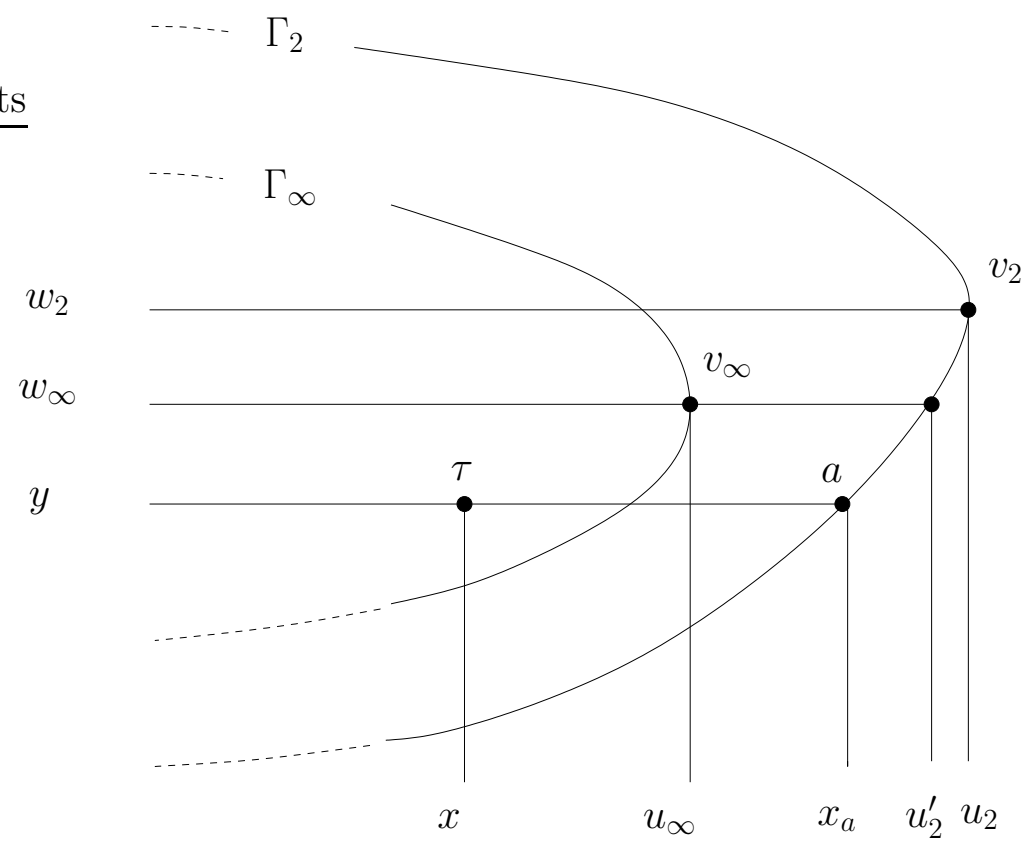

FiguRE 7.3.

We proved (1). Next we will prove (4). First the upper bound. To do so, use the inequalities (7.10), (7.11), (7.13), and (7.15) to estimate the four term below.

$$
\begin{aligned}
|\tau-a| & =\left|x-x_{a}\right| \\
& \leq\left|x-u_{\infty}\right|+\left|u_{\infty}-u_{2}^{\prime}\right|+\left|u_{2}^{\prime}-u_{2}\right|+\left|u_{2}-x_{a}\right| \\
& =O\left(b_{F}\right) .
\end{aligned}
$$

The lower bound follows from inequalities (7.11), (7.13), and (7.15):

$$
\begin{aligned}
|\tau-a| & \geq\left|x_{a}-u_{\infty}\right| \\
& \geq\left|u_{2}^{\prime}-u_{\infty}\right|-\left|u_{2}-u_{2}^{\prime}\right|-\left|u_{2}-x_{a}\right| \\
& =O\left(b_{F}\right) .
\end{aligned}
$$

Left is to prove (3). To do so, use the inequalities (7.16), (7.15), and (7.14):

$$
\left|\tau-v_{2}\right| \leq|\tau-a|+\sqrt{\left|x_{a}-u_{2}\right|^{2}+\left|y-w_{2}\right|^{2}}=O\left(b_{F}\right) .
$$

\section{The AVERAge JaCOBIAN AS TOPOLOGICAL INVARIANT}

Fix $F \in \mathcal{I}_{\Omega}(\bar{\varepsilon})$ with $\bar{\varepsilon}>0$ small enough. Let $M_{1}^{k} \subset B$, with $k \geq 1$, be as defined in $₫ 3$, Let $D^{\tau} \subset D_{1}$ be the connected component of 
$D_{1} \backslash W_{\text {loc }}^{s}(\tau)$ which does not contain $\beta_{1}$, see Figure 8.1. Define,

$$
\kappa_{F}=\min \left\{k \geq 1 \mid M_{1}^{k} \cap D^{\tau} \neq \emptyset\right\} .
$$

Lemma 8.1. Let $F, \tilde{F} \in \mathcal{I}_{\Omega}(\bar{\varepsilon})$ with $\bar{\varepsilon}>0$ small enough. If $F$ and $\tilde{F}$ are conjugate then

$$
\kappa_{\tilde{F}}=\kappa_{F} .
$$

Moreover, if $h$ is a conjugation between $F$ and $\tilde{F}$ with $h\left(D_{1}\right)=\tilde{D}_{1}$ then

$$
h\left(D^{\tau} \cap M_{1}^{k}\right)=\tilde{D}^{\tau} \cap \tilde{M}_{1}^{k} .
$$

Proof. The curves $M_{1}^{k}$ are not topologically determined. However,

$$
M_{1}^{k} \subset F^{-2^{k+1}}\left(\left[p_{0}^{k}, p_{2}^{k}\right]^{s}\right)
$$

where $\left[p_{0}^{k}, p_{2}^{k}\right]^{s} \subset W^{s}\left(\beta_{k}\right)$ is the curve connecting $p_{0}^{k}$ with $p_{2}^{k}$, see $₫ 3$ and Figure 3.1. Proposition 3.5 gives

$$
D^{\tau} \cap F^{-2^{k+1}}\left(\left[p_{0}^{k}, p_{2}^{k}\right]^{s}\right)=D^{\tau} \cap M_{1}^{k} .
$$

We may assume, see Proposition 5.2, that we have a conjugation $h$ between $F$ and $\tilde{F}$ with $h\left(D_{1}\right)=\tilde{D}_{1}$. The set $D^{\tau} \cap F^{-2^{k+1}}\left(\left[p_{0}^{k}, p_{2}^{k}\right]^{s}\right)$ is topologically defined. So,

$$
\begin{aligned}
h\left(D^{\tau} \cap M_{1}^{k}\right) & =h\left(D^{\tau} \cap F^{-2^{k+1}}\left(\left[p_{0}^{k}, p_{2}^{k}\right]^{s}\right)\right) \\
& =\tilde{D}^{\tau} \cap \tilde{F}^{-2^{k+1}}\left(\left[\tilde{p}_{0}^{k}, \tilde{p}_{2}^{k}\right]^{s}\right) \\
& =\tilde{D}^{\tau} \cap \tilde{M}_{1}^{k} .
\end{aligned}
$$

This means that $\kappa_{F}$ is a topological invariant.

We will suppress the index $F: \kappa=\kappa_{F}$ and $b=b_{F}$, etc. The intersection point of $M_{1}^{k}$ with the horizontal line through $\tau$ is denoted by $z_{k}$.

Remark 8.1. The proof of Proposition 8.2 is illustrated in Figure 8.1. Again the horizontal direction has been stretched dramatically.

\section{Proposition 8.2.}

$$
\left|\tau-z_{\kappa}\right| \asymp b .
$$

Proof. The curves $M_{1}^{k}$ are graphs over the $y$-axis with a slope bounded by

$$
\left|\frac{d M_{1}^{k}}{d y}\right| \leq K_{1} \cdot b
$$

Consider the curve $\Gamma_{2}$, as defined in 97] This curve has a definite curvature, see Lemma 7.3. This means that there are unique tangent lines $L_{1}$ and $L_{2}$ to $\Gamma_{2}$ with slopes, as a graph of the $y$-axis, equal to $-K_{1} \cdot b$ and $K_{1} \cdot b$. The connected component of $B \backslash\left(L_{1} \cup L_{2}\right)$ which 


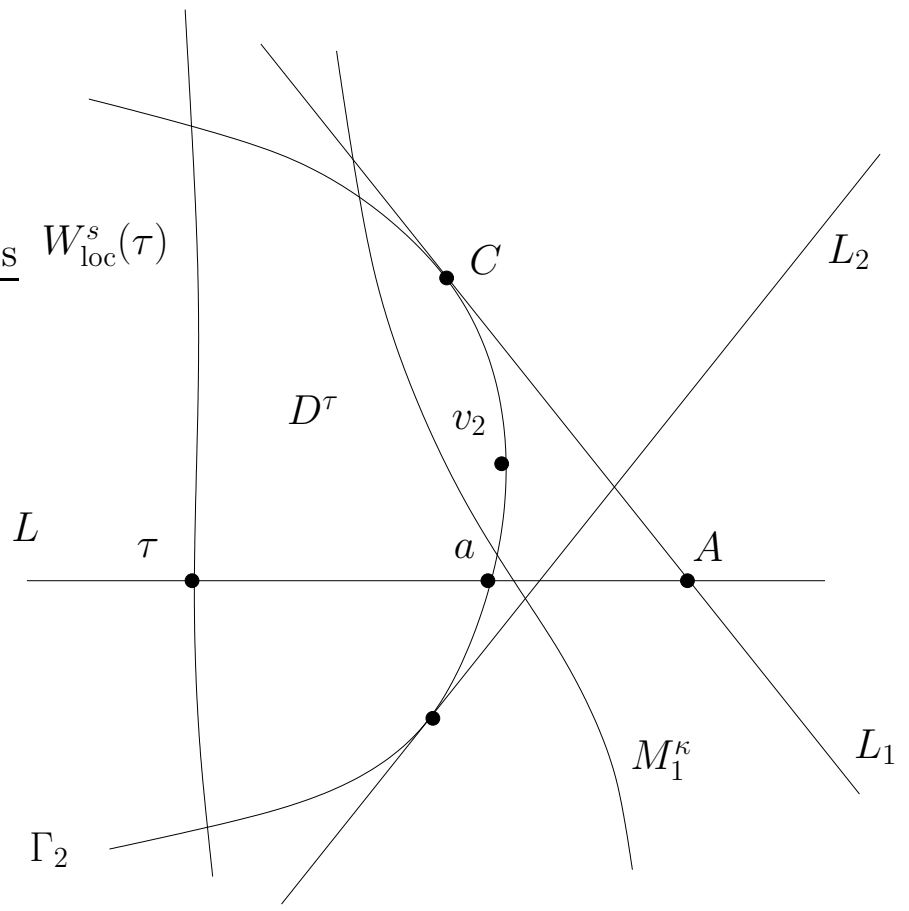

FIGURE 8.1.

contains $D_{\tau}$ is called $D$. The lines $L_{1}$ and $L_{2}$ intersect the horizontal line $L$ trough $\tau$. Let $A \in L$ be among these intersection points the one furthest to the right, say $A=L \cap L_{1}$. Denote the tangent points of $L_{1}$ by $C$. Finally, let $(A, \infty) \subset L$ be the connected component of $L \backslash\{A\}$ which does not contain $\tau$.

Observe, every curve intersecting $D$ and $(A, \infty)$ will have somewhere a tangent line with absolute value of the slope, with respect to the $y$ axis, larger than $K_{1} \cdot b$. Hence,

$$
M_{1}^{k} \cap D^{\tau} \neq \emptyset \Rightarrow M_{1}^{k} \cap[\tau, A] \neq \emptyset .
$$

The intersection point of $\Gamma_{2}$ with the horizontal line through $\tau$ is denoted by $a$. Then

$$
M_{1}^{k} \cap[\tau, a] \neq \emptyset \Rightarrow M_{1}^{k} \cap D^{\tau} \neq \emptyset .
$$

The implication (8.2) and Proposition [7.4(4) imply

$$
\left|\tau-z_{\kappa-1}\right| \geq|\tau-a| \asymp b .
$$

Proposition 3.5 states

$$
\left|\tau-z_{k}\right| \asymp \sigma^{2 k} .
$$


Then inequality (8.3) and (8.4) give $K_{2}>0$ such that

$$
\left|\tau-z_{\kappa}\right| \geq K_{2} \cdot b \text {. }
$$

An upper bound is given by (8.1). Namely,

$$
\left|\tau-z_{\kappa}\right| \leq|\tau-A| \text {. }
$$

Left is to estimate $|\tau-A|$. According to Lemma 7.3 and Proposition $7.4(1)$ and (2) we get

$$
\begin{aligned}
& \left|\pi_{2}(a)-\pi_{2}\left(v_{2}\right)\right|=O(b), \\
& \left|\pi_{1}(a)-\pi_{1}\left(v_{2}\right)\right|=O\left(b^{2}\right) .
\end{aligned}
$$

Lemma 7.3 implies

$$
\begin{aligned}
& \left|\pi_{2}(C)-\pi_{2}\left(v_{2}\right)\right|=O(b), \\
& \left|\pi_{1}(C)-\pi_{1}\left(v_{2}\right)\right|=O\left(b^{2}\right) .
\end{aligned}
$$

Hence,

$$
\begin{aligned}
& \left|\pi_{2}(C)-\pi_{2}(a)\right|=O(b), \\
& \left|\pi_{1}(C)-\pi_{1}(a)\right|=O\left(b^{2}\right) .
\end{aligned}
$$

The slope of $L$ with the vertical is $\pm K_{1} \cdot b$. Hence,

$$
\left|\pi_{1}(C)-\pi_{1}(A)\right|=O\left(b^{2}\right) .
$$

The estimates (8.7) and (8.8) imply,

$$
|a-A|=O\left(b^{2}\right) \text {. }
$$

Together with Proposition 7.4(4) we get

$$
|\tau-A|=|\tau-a|+|a-A| \asymp b .
$$

Thus together with (8.5) and (8.6) we get

$$
\left|\tau-z_{\kappa}\right| \asymp b \text {. }
$$

Theorem 8.3. Let $F \in \mathcal{I}_{\Omega}(\bar{\varepsilon})$, with $\bar{\varepsilon}>0$ small enough. Then

$$
\boldsymbol{\kappa}_{F}=\lim _{n \rightarrow \infty} \frac{\kappa_{R^{n} F}}{2^{n}}=\frac{1}{2} \frac{\ln b_{F}}{\ln \sigma}
$$

exists and is a topological invariant. In particular, $b_{F}$ is a topological invariant.

Proof. If $F, \tilde{F} \in \mathcal{I}_{\Omega}(\bar{\varepsilon})$ are conjugate then there is a conjugation $h$ between them with

$$
h\left(D_{n}\right)=\tilde{D}_{n}
$$


$n \geq 0$. This follows from Lemma 5.2 and Proposition 5.3. This means that the conjugation $h$ induces a conjugation, denoted by $h_{n}$, between $F_{n}=R^{n} F$ and $\tilde{F}_{n}=R^{n} \tilde{F}$. Thus

$$
h_{n}\left(F_{n}^{-2^{k+1}}\left(\left[p_{0}^{k}, p_{2}^{k}\right]^{s}\right)\right)=\tilde{F}_{n}^{-2^{k+1}}\left(\left[\tilde{p}_{0}^{k}, \tilde{p}_{2}^{k}\right]^{s}\right)
$$

and

$$
h_{n}\left(D^{\tau}\left(F_{n}\right)\right)=D^{\tau}\left(\tilde{F}_{n}\right) .
$$

This implies

$$
\kappa_{\tilde{F}_{n}}=\kappa_{F_{n}} .
$$

The definition of $\boldsymbol{\kappa}_{F}$ is topological. Left is to identify its value. Proposition 3.5 gives

$$
\left|\tau_{F_{n}}-z_{\kappa_{F_{n}}}\right| \asymp \sigma^{2 \kappa_{F_{n}}}
$$

and Proposition 8.2 gives

$$
\left|\tau_{F_{n}}-z_{\kappa_{F_{n}}}\right| \asymp b^{2^{n}} .
$$

These two estimates imply

$$
\sigma^{2 \kappa_{F_{n}}} \asymp b^{2^{n}} .
$$

Thus

$$
\boldsymbol{\kappa}_{F}=\lim _{n \rightarrow \infty} \frac{\kappa_{F_{n}}}{2^{n}}=\frac{1}{2} \frac{\ln b_{F}}{\ln \sigma} .
$$

\section{The STABLE LAMiNATION}

Let $F \in \mathcal{H}_{\Omega}^{n}(\bar{\varepsilon})$, with $\bar{\varepsilon}>0$ small enough. The set $\mathcal{P}_{F}^{n}$ consists of the periodic points of period at most $2^{n-1}$. Define

$$
\mathcal{F}_{n}^{s}=\bigcup_{x \in \mathcal{P}_{F}^{n}} W^{s}(x) .
$$

Lemma 9.1. $\mathcal{F}_{n}^{s}$ is closed.

Proof. Let $z_{j} \in \mathcal{F}_{n}^{s}$ with $z_{j} \rightarrow z$. We may assume that for all $j \geq 1$ $z_{j} \in W^{s}\left(\beta_{k}\right)$ with $k \leq n$. Suppose $z \notin \mathcal{F}_{n}^{s}$. According to Theorem 4.1 we have

$$
\omega(z) \subset \bigcup_{k>n} \boldsymbol{\beta}_{k} \cup \mathcal{O}_{F} \subset \operatorname{int}\left(\operatorname{Trap}_{n+1}\right) .
$$

$\operatorname{Trap}_{n+1}$ is forward invariant. Hence, for $j \geq 1$ large enough,we have

$$
\boldsymbol{\beta}_{k}=\omega(z) \subset \operatorname{Trap}_{n+1} .
$$

Contradiction, $k \leq n$. 
Remark 9.1. For $x \in \mathcal{P}_{F}^{n}$

$$
\overline{\operatorname{Orb}\left(W^{s}(x)\right)} \backslash \operatorname{Orb}\left(W^{s}(x)\right)=\mathcal{F}_{n-1}^{s} .
$$

The proof of the fact that the closure is contained in $\mathcal{F}_{n-1}^{s}$ relies on Lemma 4.7 and the proof of Lemma 9.1. The other inclusion follows from a statement similar to Lemma 4.4 but discussing stable manifolds.

A point $z \in \mathcal{F}_{n}^{s}$ is laminar if for any sequence $z_{j} \in \mathcal{F}_{n}^{s}$ with $z_{n} \rightarrow z$

$$
T_{z_{j}} W^{s}\left(z_{j}\right) \rightarrow T_{z} W^{s}(z) .
$$

The set $\mathcal{F}_{n}^{s}$ is laminar if all its points are laminar.

Theorem 9.2. Let $F \in \mathcal{H}_{\Omega}^{n}(\bar{\varepsilon})$, with $\bar{\varepsilon}>0$ small enough. If

$$
F \notin \bigcup_{k^{\prime}<n^{\prime} \leq n} \mathcal{K}_{k^{\prime}, n^{\prime}}
$$

then $\mathcal{F}_{n}^{s}$ is laminar.

The proof of this Theorem is similar to the proof of Theorem4.6 with some modifications, see the proof of Claim 9.4. For completeness we include the proof. Using the notation of $\$ 4$ we will choose the interval

$$
K_{n}^{s}=\left[p_{0}, p_{2}\right]^{s} \subset W_{\mathrm{loc}}^{s}\left(\beta_{n}\right),
$$

as a fundamental domain in $W^{s}\left(\beta_{n}\right)$, see Figure 4.1. For $k<n$ define,

$$
E_{n, k}^{s}=\left\{x \in K_{n}^{s} \mid \exists t>0 \forall j<t F^{-j}(x) \notin T_{k} \text { and } F^{-t}(x) \in T_{k}\right\} .
$$

The time $t>0$ in the above definition is called the time of entry of $x \in E_{n, k}^{s}$ into $T_{k}$.

Definition 9.1. Let $k<n$. We say that $F$ satisfies the transversality condition $\mathcal{T}_{n, k}^{s}$ if the following holds. Let $z_{j} \in E_{n, k}^{s}, j \geq 0$, be a sequence such that

$$
F^{-t_{j}}\left(z_{j}\right) \rightarrow u \in U_{k}
$$

where $t_{j}>0$ is the time of entry of $z_{j}$ into $T_{k}$, then

$$
D F^{-t_{j}}\left(z_{j}\right)\left(T_{z_{j}} W^{s}\left(\beta_{n}\right)\right) \nrightarrow T_{u} W^{u}\left(\beta_{k}\right) .
$$

Proposition 9.3. Let $F \in \mathcal{H}_{\Omega}^{n}(\bar{\varepsilon}), \bar{\varepsilon}>0$ small enough. Let $k<n$ and

$$
F \notin \bigcup_{k \leq k^{\prime}<n^{\prime} \leq n} \mathcal{K}_{k^{\prime}, n^{\prime}}(\bar{\varepsilon})
$$

then $\mathcal{T}_{n, k}^{s}$ holds. 
Proof. Choose a sequence $z_{j} \in E_{n, k}^{s}, j \geq 0$, with $z_{j} \rightarrow z$ and

$$
F^{-t_{j}}\left(z_{j}\right) \rightarrow u \in \operatorname{int}\left(U_{n}\right)
$$

where $t_{j}>0$ is the time of entry of $z_{j}$ into $T_{k}$. If the $t_{j}$ are bounded, say constant $t_{j}=t$, the absence of heteroclinic tangencies, $F \notin \mathcal{K}_{k, n}(\bar{\varepsilon})$, implies that

$$
D F^{-t}(z)\left(T_{z} W^{s}\left(\beta_{k}\right)\right) \neq T_{u} U_{k}
$$

Hence,

$$
D F^{-t_{j}}\left(z_{j}\right)\left(T_{z_{j}} W^{s}\left(\beta_{k}\right)\right) \nrightarrow T_{u} U_{k} .
$$

Let us continue with the case when $t_{j} \rightarrow \infty$.

Claim 9.4. There exists $n>m_{1} \geq k$ such that $z \in E_{n, m_{1}}^{s} \cap W^{u}\left(\boldsymbol{\beta}_{m_{1}}\right)$.

Proof. Let $t \geq 0$ be an arbitrary moment of time. Assume

$$
F^{-t}(z) \notin \operatorname{Trap}_{k} \text {. }
$$

This means that for $j \geq 1$ large enough,

$$
F^{-t}\left(z_{j}\right) \notin \operatorname{Trap}_{k},
$$

because $\operatorname{Trap}_{k}$ is closed. The invariance $F\left(\operatorname{Trap}_{k}\right) \subset \operatorname{Trap}_{k} \operatorname{implies~that~}$ for all $j \geq 1$

$$
F^{-t_{j}}\left(z_{j}\right) \notin \operatorname{Trap}_{k} .
$$

The construction of $\operatorname{Trap}_{k}$ implies that

$$
F^{2^{k}}\left(\operatorname{Trap}_{k}\right) \subset \operatorname{int}\left(\operatorname{Trap}_{k}\right) .
$$

From (9.1) and (9.2) we get

$$
F^{-t_{j}}\left(z_{j}\right) \rightarrow u \notin \operatorname{Trap}_{k} .
$$

Contradiction. So for each $t \geq 0$

$$
F^{-t}(z) \in \operatorname{Trap}_{k} \text {. }
$$

This means

$$
\alpha(z) \subset \operatorname{Trap}_{k} .
$$

Suppose,

$$
\alpha(z) \cap \operatorname{Trap}_{n+1} \neq \emptyset .
$$

Then (9.2) implies that

$$
\alpha(z) \cap \operatorname{int}\left(\operatorname{Trap}_{n+1}\right) \neq \emptyset .
$$

So there is a $t>0$ such that $F^{-t}(z) \in \operatorname{Trap}_{n+1}$. Again, (9.2) implies

$$
\boldsymbol{\beta}_{n}=\omega(z) \subset \operatorname{Trap}_{n+1} .
$$

Contradiction. Thus

$$
\alpha(z) \cap \operatorname{Trap}_{n+1}=\emptyset
$$


According to Theorem 4.1 every non periodic orbit outside Trap $_{n+1}$ will enter $\operatorname{Trap}_{n+1}$. Hence, for some $m_{1} \leq n$

$$
\alpha(z)=\boldsymbol{\beta}_{m_{1}} .
$$

In particular,

$$
z \in W^{u}\left(\boldsymbol{\beta}_{m_{1}}\right)
$$

There are no homoclinic orbits. Hence, $m_{1}<n$.

Left is to show that $k \leq m_{1}$. Observe, $\alpha(z) \subset \operatorname{Trap}_{k}$, see (9.3), and $\alpha(z)=\boldsymbol{\beta}_{m_{1}}$. If $m_{1}<k$ then $\boldsymbol{\beta}_{m_{1}} \cap \operatorname{Trap}_{k}=\emptyset$. So $k \leq m_{1}<n$, which finishes the proof of the Claim.

Denote the time of entry of $z$ into $U_{m_{1}} \subset T_{m_{1}}$ by $r_{1}>0$ and let $F^{-r_{1}}(z)=u_{1}$. We will call $r_{1}$ the first transient time. For $j>0$ large enough, $z_{j} \in E_{n, m_{1}}^{s}$ with corresponding time of entry $t_{j}^{1}=r_{1}$. The absence of heteroclinic tangencies, $F \notin \mathcal{K}_{m_{1}, n}(\bar{\varepsilon})$, implies that

$$
D F^{-r_{1}}(z)\left(T_{z} W^{s}\left(\beta_{n}\right)\right) \neq T_{u_{1}} U_{m_{1}} \text {. }
$$

Hence,

$$
D F^{-t_{j}^{1}}\left(z_{j}\right)\left(T_{z_{j}} W^{s}\left(\beta_{n}\right)\right) \nrightarrow T_{u_{1}} U_{m_{1}} .
$$

In the case when $m_{1}=k$ we proved that the sequence $z_{j}$ satisfies the transversality condition.

Consider the case when $m_{1}>k$. Let $e_{j}^{1}>0$ be such that for $i=$ $r_{1}, r_{1}+1, \ldots, e_{j}^{1}$

but

$$
F^{-i}\left(z_{j}\right) \in T_{m_{1}}
$$

$$
F^{-\left(e_{j}^{1}+1\right)}\left(z_{j}\right) \notin T_{m_{1}}
$$

The moment $e_{j}^{1}$ is called the time of exit of $z_{j}$ from $T_{m_{1}}$. We may assume that $F^{-e_{j}^{1}}\left(z_{j}\right) \rightarrow s_{1} \in S_{m_{1}}$. Then (9.4) implies

$$
D F^{-e_{j}^{1}}\left(z_{j}\right)\left(T_{z_{j}} W^{s}\left(\beta_{k}\right)\right) \rightarrow T_{s_{1}} S_{m_{1}} .
$$

Now, we can repeat the proof of Claim 9.4 and obtain $k \leq m_{2}<$ $m_{1}<n$ and $r_{2}>0$, the second transient time, such that

$$
F^{-r_{2}}\left(s_{1}\right)=u_{2} \in U_{m_{2}} .
$$

For $j>0$ large enough we have $z_{j} \in E_{n, m_{2}}^{s}$. Denote the time of entry of $z_{j}$ into $T_{m_{2}}$ by $t_{j}^{2}>0$ then $t_{j}^{2}=e_{j}^{1}+r_{2}$. The absence of heteroclinic tangencies, $F \notin \mathcal{K}_{m_{2}, m_{1}}(\bar{\varepsilon})$, implies that

$$
D F^{-r_{2}}\left(s_{1}\right)\left(T_{s_{1}} W^{s}\left(\beta_{m_{1}}\right)\right) \neq T_{u_{2}} U_{m_{2}} .
$$

Hence, (9.5) implies

$$
D F^{-t_{j}^{2}}\left(z_{j}\right)\left(T_{z_{j}} W^{s}\left(\beta_{n}\right)\right) \nrightarrow T_{u_{2}} U_{m_{2}} .
$$


Let $e_{j}^{2}>0$ be maximal such that for $i=t_{j}^{2}, t_{j}^{2}+1, \ldots, e_{j}^{2}$

$$
F^{-i}\left(z_{j}\right) \in T_{m_{2}} \text {. }
$$

but

$$
F^{-\left(e_{j}^{2}+1\right)}\left(z_{j}\right) \notin T_{m_{2}} .
$$

We may assume that $F^{-e_{j}^{2}}\left(z_{j}\right) \rightarrow s_{2} \in S_{m_{2}}$. Then

$$
D F^{-e_{j}^{2}}\left(z_{j}\right)\left(T_{z_{j}} W^{s}\left(\beta_{n}\right)\right) \rightarrow T_{s_{2}} S_{m_{2}} .
$$

If $m_{2}=k$, statement (9.6) proves the transversality property. In the case when $m_{2}>k$ we can repeat this construction, and we get a sequence $m_{1}>m_{2}>m_{3}>\cdots>m_{g}$ together with points $u_{l} \in U_{m_{l}}$, $s_{l} \in S_{m_{l}}$ and entry and exit times $t_{j}^{l}>0$ and $e_{j}^{l}>0$ for $z_{j} \in E_{n, m_{l}}^{s}$ and the corresponding asymptotic expressions (9.4) and (9.5).

The sequence $m_{l}$ is strictly decreasing. Hence, $m_{g}=k$ and $t_{j}=t_{j}^{g}$ for some $g \geq 1$. Now, statement (9.4) corresponding to $T_{m_{g}}$,

$$
D F^{-t_{j}}\left(z_{j}\right)\left(T_{z_{j}} W^{s}\left(\beta_{k}\right)\right) \nrightarrow T_{u} U_{k},
$$

finishes the proof of the Proposition.

Proof of Theorem 9.2. Choose $k \leq n$. To prove that every point in $W^{s}\left(\beta_{k}\right)$ is laminar it suffices to prove that every point $z \in S_{k}$ is laminar. From Lemma 4.7 we have that $W^{s}\left(\beta_{k}\right)$ is an embedded onedimensional manifold. Hence, the only non-trivial accumulation is from $W^{u}\left(\beta_{k^{\prime}}\right)$ with $n \geq k^{\prime}>k$. Let $k^{\prime}>k$ and $z_{j} \in E_{k^{\prime}, k}^{s}$ be a sequence with

$$
F^{-t_{j}}\left(z_{j}\right) \rightarrow u \in U_{k},
$$

and

$$
F^{-e_{j}}\left(z_{j}\right) \rightarrow z \in S_{k}
$$

with $e_{j}>t_{j}$.

Proposition 9.3 states that $\mathcal{T}_{k^{\prime}, k}^{s}$ holds. Now $\mathcal{T}_{k^{\prime}, k}^{s}$ implies that

$$
D F^{-t_{j}}\left(z_{j}\right)\left(T_{z_{j}} W^{s}\left(\beta_{k^{\prime}}\right)\right) \nrightarrow T_{u} U_{k} .
$$

Hence, according to the $\lambda$-Lemma,

$$
D F^{-e_{j}}\left(z_{j}\right)\left(T_{z_{j}} W^{s}\left(\beta_{k^{\prime}}\right)\right) \rightarrow T_{z} S_{k} .
$$

The proof of the following Theorem is similar to the proof of Theorem 6.1. We will omit the proof. For a $F \in \mathcal{H}_{\Omega}^{n}(\bar{\varepsilon})$ let $\mathcal{C}_{n}^{s} \subset \mathcal{F}_{n}^{s}$ be the set of non-laminar points of $\mathcal{F}_{n}^{s}$. The stable and unstable eigenvalues of $\beta_{k}$ are denoted by $\lambda_{k}$ and $\mu_{k}$, see $₫ 6$. 
Theorem 9.5. If $F \in \mathcal{H}_{\Omega}^{n}(\bar{\varepsilon})$, with $\bar{\varepsilon}>0$ small enough, has an $(k, n)$ heteroclinic tangency with $k<n$ and

$$
\frac{\ln \left|\lambda_{k}\right|}{\ln \left|\mu_{n}\right|} \notin \mathbb{Q}
$$

then

$$
\mathcal{F}_{k}^{s} \subset \mathcal{C}_{n}^{s}
$$

\section{Morse-Smale COMPonents}

A map $F: B \rightarrow B$ is Morse-Smale if the non-wandering set $\Omega_{F}$ consists of finitely many periodic points, all hyperbolic, and the stable and unstable manifolds of the periodic points are all transversal to each other. Recall, the collection $\mathcal{I}_{\Omega}^{n}(\bar{\varepsilon})$ consists of the maps which are exactly $n$-times renormalizable and has a periodic attractor of period $2^{n}$. According to Lemma 4.5 the non-wandering set of each map $F \in$ $\mathcal{I}_{\Omega}^{n}(\bar{\varepsilon})$, with $\bar{\varepsilon}>0$ small enough, consists of finitely many periodic points. In particular, a map $F \in \mathcal{I}_{\Omega}^{n}(\bar{\varepsilon})$ is Morse-Smale if all its periodic points are hyperbolic and if for every $x, y \in \mathcal{P}_{F}=\Omega_{F}$ there are only transverse intersections of $W^{u}(x)$ and $W^{s}(y)$.

Theorem 10.1. Let $\bar{\varepsilon}>0$ be small enough. The Morse-Smale maps form an open and dense subset of any $\mathcal{I}_{\Omega}^{n}(\bar{\varepsilon})$.

A Morse-Smale component is a connected component of the set of non-degenerate Morse-Smale maps in $\mathcal{H}_{\Omega}(\bar{\varepsilon})$. Morse-Smale maps are structurally stable, see $[\mathrm{P}]$.

Proposition 10.2. Let $F, \tilde{F} \in \mathcal{I}_{\Omega}^{n}(\bar{\varepsilon})$, with $\bar{\varepsilon}>0$ small enough, be in the same Morse-Smale component. Then $F$ and $\tilde{F}$ are conjugate.

Two Morse-Smale components in $\mathcal{I}_{\Omega}^{n}(\bar{\varepsilon})$ are of different type if the maps in the first component are not conjugate to the maps in the other.

Remark 10.1. In this discussion we will only consider non-degenerate Hénon maps. Observe, if $F \in \mathcal{I}_{\Omega}^{n}(\bar{\varepsilon})$ is a unimodal map, it can be of three different topological types depending the relative position of the attracting fixed point $p$ and the critical point $c$ of the unimodal map which describes the $n^{t h}$-renormalization: $p<c, p=c$, and $p>$ $c$. The non-degenerate Hénon maps in the Morse-Smale component which contains perturbations of the unimodal maps in $F \in \mathcal{I}_{\Omega}^{n}(\bar{\varepsilon})$ are all conjugated. There is no difference in the topology of the periodic attractor anymore. 
Theorem 10.3. Let $\bar{\varepsilon}>0$ be small enough. Then for $n \geq 1$ large enough there are countably many Morse-Smale components of different type in $\mathcal{I}_{\Omega}^{n}(\bar{\varepsilon})$.

There are a non-locally finite collections of bifurcation curves in Hénon-families. Some of these collections are constructed in the proof of Theorem 10.3, they are illustrated in Figure 10.1.

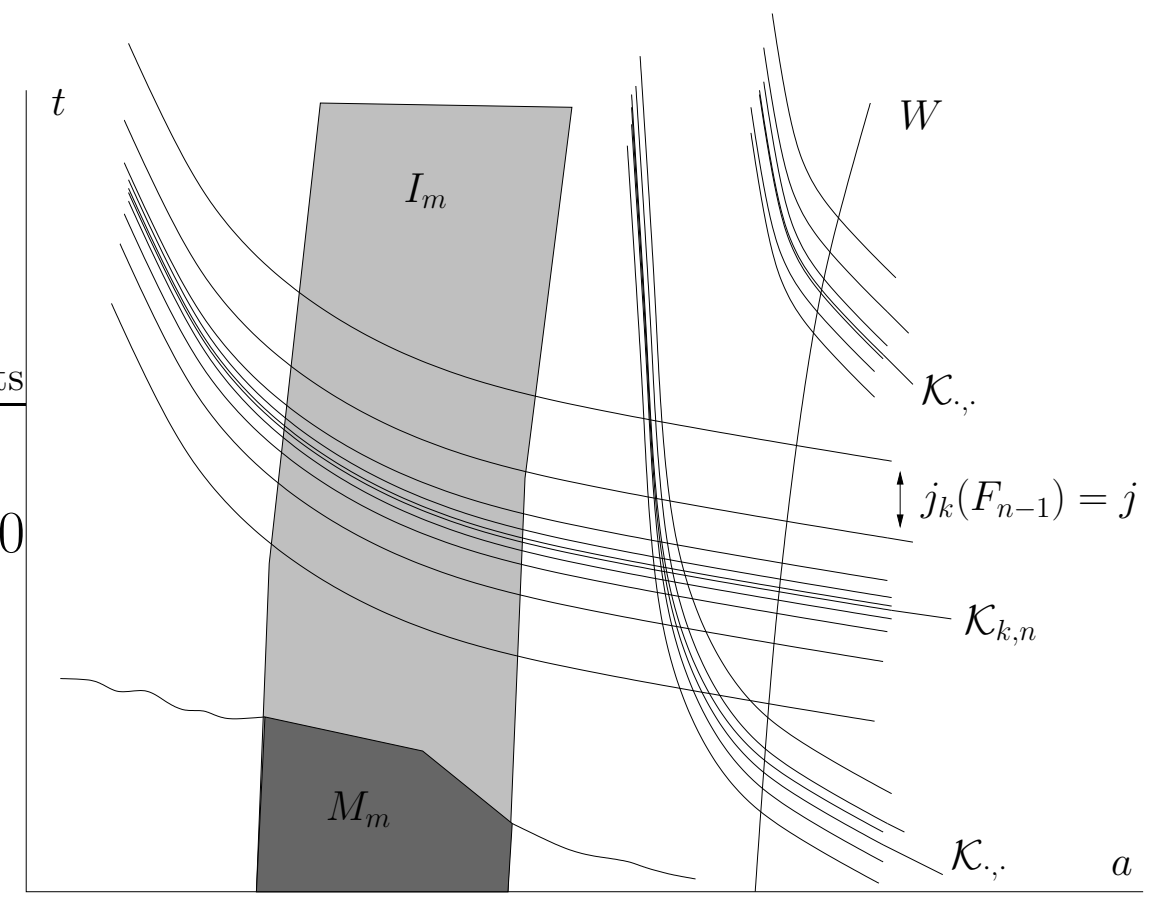

FIGURE 10.1. Bifurcation pattern

The actual proofs of these Theorems need some preparation. Recall,

$$
\mathcal{U} \mathcal{K}_{k, n}(\bar{\varepsilon})=\bigcup_{k \leq k^{\prime}<n^{\prime} \leq n} \mathcal{K}_{k^{\prime}, n^{\prime}}(\bar{\varepsilon}) .
$$

Lemma 10.4. If $k<n$ and $\bar{\varepsilon}>0$ small enough then

$$
\overline{\mathcal{K}_{k, n}(\bar{\varepsilon})} \subset \mathcal{U} \mathcal{K}_{k, n}(\bar{\varepsilon}) \text {. }
$$

In particular, $\mathcal{U K}_{k, n}(\bar{\varepsilon})$ is closed.

Proof. It suffices to prove that for each $k<n$

$$
\overline{\mathcal{K}_{k, n}(\bar{\varepsilon})} \subset \mathcal{U} \mathcal{K}_{k, n}(\bar{\varepsilon}) .
$$

Let $F_{j} \in \mathcal{K}_{k, n}(\bar{\varepsilon})$ with $F_{j} \rightarrow F \notin \mathcal{U} \mathcal{K}_{k, n}(\bar{\varepsilon})$. Let $K_{m}^{u / s} \subset W_{\text {loc }}^{u / s}\left(\beta_{m}\right)$ be a fundamental domain for $F$ restricted to $W^{u / s}\left(\boldsymbol{\beta}_{n}\right)$. We may assume that $K_{m}^{u} \subset \operatorname{int} U_{m}$ and $K_{m}^{s} \subset \operatorname{int} S_{m}$. Similarly, let $K_{m}^{u / s}(j) \subset$ 
$W_{\text {loc }}^{u / s}\left(\beta_{m}(j)\right)$ be a fundamental domain for $F_{j}$ restricted to $W^{u / s}\left(\boldsymbol{\beta}_{n}(j)\right)$. Construct these fundamental domains in such a way that

$$
K_{m}^{u / s}(j) \rightarrow K_{m}^{u / s}
$$

Apply Lemma 4.8 and we see that $K_{m}^{u}(j) \subset \operatorname{int} U_{m}(j)$ and $K_{m}^{s}(j) \subset$ int $S_{m}(j)$, for $j \geq 1$ large enough. Finally, choose $x_{j} \in K_{k}^{u}(j)$ such that

$$
F^{t_{j}}\left(x_{j}\right) \in K_{n}^{s}(j)
$$

is a heteroclinic tangency and $x_{j} \rightarrow x \in K_{k}^{u}$.

Claim 10.5. $\omega(x) \subset \mathcal{O}_{F} \cup \bigcup_{m>n} \boldsymbol{\beta}_{m}$

Proof. Suppose by contradiction $\omega(x)=\boldsymbol{\beta}_{m_{1}}$, say

$$
s_{1}=F^{r_{1}}(x) \in K_{m_{1}}^{s},
$$

with $m_{1} \leq n$. ¿From $F \notin \mathcal{K}_{k, m_{1}}(\bar{\epsilon})$ we get that

$$
T_{s_{1}} F^{r_{1}}\left(K_{k}^{u}\right) \pitchfork T_{s_{1}} K_{m_{1}}^{s} .
$$

By definition

$$
T_{x_{j}} K_{k}^{u}(j) \rightarrow T_{x} K_{k}^{u}
$$

This implies

$$
D F_{j}^{r_{1}}\left(x_{j}\right)\left(T_{x_{j}} K_{k}^{u}(j)\right) \nrightarrow T_{s_{1}} K_{m_{1}}^{s} .
$$

Now we will prove

$$
m_{1}<n .
$$

To do so, assume that $m_{1}=n$. Observe, $F^{r_{1}}\left(x_{j}\right) \in W^{s}\left(\boldsymbol{\beta}_{n}(j)\right)$ and this point is also close to $S_{n}(j)$ because it is close to $S_{n}$. Lemma 4.8 implies

$$
F_{j}^{r_{1}}\left(x_{j}\right) \in K_{n}^{s}(j) .
$$

Hence, $t_{j}=r_{1}$. From (10.2) we get that at $F_{j}^{r_{1}}\left(x_{j}\right)$ there is no tangency between $F_{j}^{r_{1}}\left(K_{k}^{u}(j)\right)$ and $K_{n}^{s}(j)$. Contradiction. We proved that $m_{1}<$ $n$.

Let $e_{j}^{1}>0$ be maximal such that when $r_{1} \leq i \leq e_{j}^{1}$ we have

$$
F_{j}^{i}\left(x_{j}\right) \in T_{m_{1}}(j)
$$

where $T_{m_{1}}(j)$ is the saddle region of $\beta_{m_{1}}(j)$ of $F_{j}$, see Figure 4.1. Say

$$
F_{j}^{e_{j}^{1}}\left(x_{j}\right) \rightarrow u_{1} \in K_{m_{1}}^{u}
$$

Then

$$
D F_{j}^{e_{j}^{1}}\left(x_{j}\right)\left(T_{x_{j}} K_{k}^{u}(j)\right) \rightarrow T_{u_{1}} K_{m_{1}}^{u}
$$


Recall, $x_{j} \in W^{s}\left(\beta_{n}(j)\right)$. Hence,

$$
\omega\left(u_{1}\right) \subset \bigcup_{m \leq n} \boldsymbol{\beta}_{m} .
$$

Say,

$$
s_{2}=F^{r_{2}}\left(u_{1}\right) \in K_{m_{2}}^{s},
$$

with $m_{1}<m_{2} \leq n$. Because, $F \notin \mathcal{K}_{m_{1}, m_{2}}(\bar{\epsilon})$ we get

$$
T_{s_{2}} F^{r_{2}} K_{m_{1}}^{u} \pitchfork T_{s_{2}} K_{m_{2}}^{s} \text {. }
$$

This implies

$$
D F_{j}^{r_{2}+e_{j}^{1}}\left(x_{j}\right)\left(T_{x_{j}} K_{k}^{u}(j)\right) \nrightarrow T_{s_{2}} K_{m_{2}}^{s} .
$$

As before, we conclude

$$
m_{1}<m_{2}<n .
$$

The statements (10.5) and (10.4) are similar to the statements (10.2) and (10.1). We can repeat the construction in a similar manner as was done in the proof of Proposition 4.10. Properties (10.3) and (10.6) show that the construction can always be repeated. This is impossible.

The first consequence of the Claim is that

$$
\mathcal{A}_{F}^{n+1} \neq \emptyset \text {. }
$$

Choose an open neighborhood $U \supset \mathcal{A}_{F}^{n+1}$ such that

$$
\overline{F(U)} \subset U
$$

and

$$
\bar{U} \cap K_{n}^{s}=\emptyset .
$$

There exists $s_{0}>0$ such that $F^{s}(x) \in U, s \geq s_{0}$. For $j \geq 1$ large enough

$$
\overline{F_{j}(U)} \subset U \text {. }
$$

Hence, for $j \geq 1$ large enough and $s \geq s_{0}$

$$
F_{j}^{s}\left(x_{j}\right) \in U \text {. }
$$

From (10.7) we get $t_{j} \leq s_{0}$. Say, $t_{j}=t$. Observe,

$$
F^{t}(x) \leftarrow F_{j}^{t}\left(x_{j}\right) \in K_{n}^{s}(j) \rightarrow K_{n}^{s} .
$$

So, $F^{t}(x) \in K_{n}^{s}$. This contradicts Claim 10.5.

Consider the set $A_{k, n} \subset K_{n}^{s}$ consisting of tangencies of $W^{u}\left(\beta_{k}\right)$ and $W^{s}\left(\beta_{n}\right)$. 
Proposition 10.6. Let $F \in \mathcal{H}_{\Omega}^{n}(\bar{\varepsilon})$, with $\bar{\varepsilon}>0$ small enough, and $k<n$ be such that $F$ has a $(k, n)$-heteroclinic tangency,

$$
F \in \mathcal{K}_{k, n}(\bar{\varepsilon})
$$

but

$$
F \notin \mathcal{U} \mathcal{K}_{k+1, n}(\bar{\varepsilon})
$$

Then $A_{k, n}$ is a finite set.

Proof. Let

$$
W=\bigcup_{j=k}^{n-1} W^{u}\left(\beta_{j}\right) .
$$

Lemma 4.9 states that the only points in $K_{n}^{s}$ on which $W^{u}\left(\beta_{k}\right)$ can accumulate are points in the unstable manifolds $W^{u}\left(\beta_{j}\right)$ with $k<j<$ $n$. Hence, if $x \in \overline{A_{k, n}} \backslash A_{k, n}$ then

$$
x \in W^{u}\left(\beta_{j}\right) \cap W^{s}\left(\beta_{n}\right)
$$

for some $j>0$ with $k<j<n$.

Condition (10.8) says that $F \notin \mathcal{K}_{j, n}$. So, the intersection at $x$ between $W^{u}\left(\beta_{j}\right)$ and $W^{s}\left(\beta_{n}\right)$ is transverse. The point $x$ is accumulated by heteroclinic tangencies. It is not a laminar point of

$$
\bigcup_{k \leq j \leq n} W^{u}\left(\boldsymbol{\beta}_{j}\right) \text {. }
$$

This contradicts Proposition 4.12,

Remark 10.2. Observe that the intersection $W \cap K_{n}^{s}$, used in the previous proof, is closed. However, it is not finite if $k<n-1$. Compare with Lemma 4.9 .

Choose $F \in \mathcal{H}_{\Omega}^{n}(\bar{\varepsilon})$, with $\bar{\varepsilon}>0$ small enough. Fix the fundamental domain $K_{n}^{s}=\left[p_{0}^{n}, p_{2}^{n}\right]^{s} \subset W_{\text {loc }}^{s}\left(\beta_{n}\right)$ for $F$ restricted to $W^{s}\left(\boldsymbol{\beta}_{n}\right)$. The points $p_{0}^{n}, p_{2}^{n}$ are as defined in section \$4 , see Figure 4.1. Similarly, we choose $K_{k}^{u}=\left[p_{-2}^{k+1}, p_{-1}^{k+1}\right]^{u}$ as a fundamental domain in $W^{u}\left(\boldsymbol{\beta}_{k}\right)$ with $k<n$. Given a sequence $F_{j} \rightarrow F$ we will denoted the fundamental domains of $F_{j}$ by $K_{k}^{u / s}(j)$. The invariant manifolds $W^{u / s}\left(\beta_{k}\left(F_{j}\right)\right)$ of $F_{j}$ will be denoted by $W_{k}^{u / s}(j)$ and $W^{u / s}\left(\beta_{k}(F)\right)$ of $F$ will be denoted by $W_{k}^{u / s}$.

Lemma 10.7. Let $F \in \mathcal{H}_{\Omega}^{n}(\bar{\varepsilon})$, with $\bar{\varepsilon}>0$ small enough, and $k<n$ be such that

$$
F \notin \mathcal{U} \mathcal{K}_{k+1, n}(\bar{\varepsilon}) \cup \mathcal{U} \mathcal{K}_{k, n-1}(\bar{\varepsilon}) .
$$


There exists $N \geq 1$ such that the following holds. If $F_{j} \rightarrow F$ and there are points

$$
\begin{gathered}
u(j) \in K_{k}^{u}(j) \\
z(j)=F_{j}^{t_{j}}(u(j))
\end{gathered}
$$

such that

$$
z(j) \rightarrow \hat{z} \in K_{n}^{s}
$$

and

$$
T_{z(j)} W_{k}^{u}(j) \ni v(j) \rightarrow T_{z} K_{n}^{s}
$$

then

$$
t_{j} \leq N
$$

Proof. Fix $k \geq 1$. The proof will be given by induction in $n>k$. The definition of renormalization implies that the Lemma holds when $n=k+1$. In this case there are only two intersections of $W_{k}^{u}(j)$ with $K_{n}^{s}(j)$ and these intersections are transversal. There is nothing to prove.

Suppose the Lemma holds for all $k+1 \leq m<n$. Suppose, $F_{j} \rightarrow F$ and this sequence satisfies the conditions (10.9), .., (10.12) of the Lemma but

$$
t_{j} \rightarrow \infty
$$

Claim 10.8. There is $k<m<n$ with $\hat{z} \in W_{m}^{u}$.

Proof. Suppose the backward orbit of $\hat{z}$ escapes from $\operatorname{Trap}_{k}(F)$ :

$$
F^{-t_{0}}(\hat{z}) \notin \operatorname{Trap}_{k}(F),
$$

for some $t_{0}>0$. Observe, $\operatorname{Trap}_{k}(F)$ is closed and $\operatorname{Trap}_{k}\left(F_{j}\right)$ is close to $\operatorname{Trap}_{k}(F)$ for $j \geq 1$ large enough. So for $j \geq 1$ large enough we have

$$
F_{j}^{-t_{0}}(\hat{z}(j)) \notin \operatorname{Trap}_{k}\left(F_{j}\right) .
$$

This contradicts, $\hat{z}(j) \in W_{k}^{u}(j)$. We showed that the backward orbit of $\hat{z}$ does not escape: for every $t \geq 0$

$$
F^{-t}(\hat{z}) \in \operatorname{Trap}_{k}(F) \text {. }
$$

Hence,

$$
\hat{z} \in \bigcup_{k \leq m<n} W_{m}^{u} .
$$

Suppose, $\hat{z} \in W_{k}^{u}$. Choose a neighborhood $U \supset \mathcal{A}_{F}^{k+1}$ such that $F(U)$ is strictly contained in $U$ and $U \cap\left[\beta_{k}, \hat{z}\right]^{u}=\emptyset$. For $j \geq$ large enough 
we have that $F_{j}$ also maps $U$ strictly inside $U$. In particular, $\mathcal{A}_{F_{j}^{k+1}}$ is strictly contained in $U$. Hence, for all $l \geq l_{0}$

$$
F_{j}^{l}(u(j)) \in U
$$

This contradicts

$$
F_{j}^{t_{j}}(u(j))=z(j) \rightarrow \hat{z} \notin U
$$

because $t_{j} \rightarrow \infty$.

Let $\hat{z}(j) \in W_{m}^{u}(j) \cap K_{n}^{s}(j)$ be the perturbation of $\hat{z} \in W_{m}^{u} \cap K_{n}^{s}$, see Figure 10.2. The intersection at $\hat{z}$ of $W_{m}^{u}$ with $K_{n}^{s}$ is transversal because $F \notin \mathcal{K}_{m, n}$ : the perturbation $\hat{z}(j)$ is well defined.

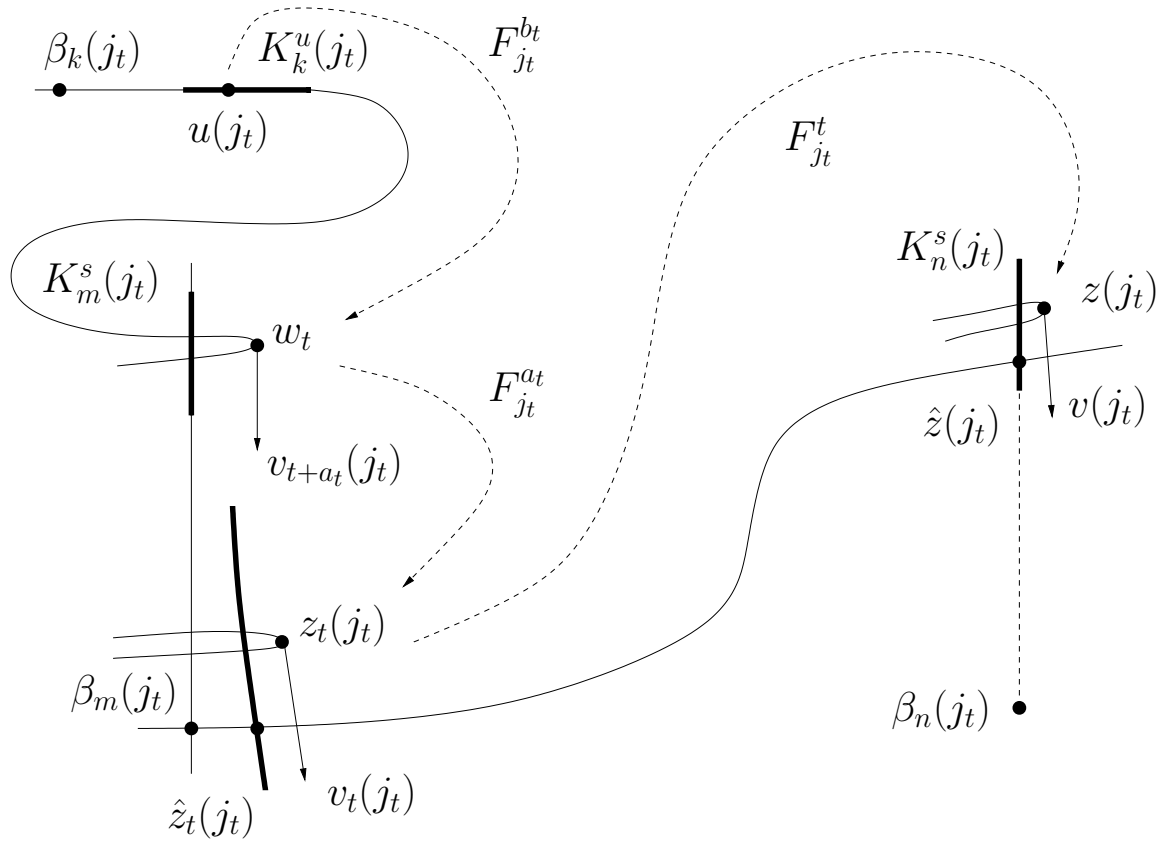

Figure 10.2.

For each $t \geq 1$ define

$$
\begin{aligned}
& \hat{z}_{t}(j)=F_{j}^{-t}(\hat{z}(j)), \\
& z_{t}(j)=F_{j}^{-t}(z(j)),
\end{aligned}
$$

and

$$
v_{t}(j)=D F_{j}^{-t}(v(j)) .
$$

From (10.12) we get for a given $t \geq 1$

$$
v_{t}(j) \rightarrow T_{\hat{z}_{t}(j)} W_{n}^{s},
$$


when $j \rightarrow \infty$. Given $t \geq 1$ we can apply the $\lambda$-Lemma, see dMP, and choose $j_{t}$ large enough and $a_{t} \geq 1$ such that

$$
w_{t}=z_{t+a_{t}}\left(j_{t}\right) \rightarrow w \in K_{m}^{s}
$$

and

$$
v_{t+a_{t}}\left(j_{t}\right) \rightarrow T_{w} K_{m}^{s}
$$

The time needed to go from $u\left(j_{s}\right)$ to $w_{s}$ is

$$
b_{t}=t_{j_{t}}-\left(t+a_{t}\right) .
$$

The induction hypothesis, that is, the Lemma for $k<m<n$ gives a bound $N \geq 1$ such that

$$
b_{t}<N
$$

for all $t \geq 1$. This implies that $w \in K_{m}^{s}$ is a tangency between $W_{k}^{u}$ and $W_{m}^{s}$. This is impossible because

$$
F \notin \mathcal{K}_{k, m} \subset \mathcal{U} \mathcal{K}_{k, n-1}
$$

Let $F \in \mathcal{H}_{\Omega}^{n}(\bar{\varepsilon})$, with $\bar{\varepsilon}>0$ small enough, and $k<n$. For $t \geq 1$ define the curve

$$
C_{k, t} \equiv\left[\beta_{k}, F^{t}\left(p_{0}^{k+1}\right)\right]^{u},
$$

the intersection point $p_{0}^{k+1}$ of $W^{u}\left(\beta_{k}\right)$ with $W^{s}\left(\beta_{k+1}\right)$ is defined in $\S 3$. See also Figure 4.1. The following Theorem is a reformulation of Lemma 10.7.

Theorem 10.9. Let $F \in \mathcal{H}_{\Omega}^{n}(\bar{\varepsilon})$, with $\bar{\varepsilon}>0$ small enough, and $k<n$ be such that

$$
F \notin \mathcal{U} \mathcal{K}_{k+1, n}(\bar{\varepsilon}) \cup \mathcal{U} \mathcal{K}_{k, n-1}(\bar{\varepsilon}) .
$$

There exists $N \geq 1$ and a neighborhood $\mathcal{U}$ of $F \in \mathcal{H}_{\Omega}^{n}(\bar{\varepsilon})$ such that the following holds. If $\tilde{F} \in \mathcal{U}$ and

$$
W^{u}\left(\beta_{k}(\tilde{F})\right) \Pi_{x} W^{s}\left(\beta_{n}(\tilde{F})\right)
$$

with $x \in K_{n}^{s}(\tilde{F})$ then

$$
x \in C_{k, N}(\tilde{F}) .
$$

Lemma 10.10. Let $F \in \mathcal{H}_{\Omega}^{n}(\bar{\varepsilon})$, with $\bar{\varepsilon}>0$ small enough, and $k<n$, be such that $F$ has a $(k, n)$-heteroclinic tangency,

$$
F \in \mathcal{K}_{k, n}(\bar{\varepsilon})
$$

but

$$
F \notin \mathcal{U} \mathcal{K}_{k+1, n}(\bar{\varepsilon}) \cup \mathcal{U} \mathcal{K}_{k, n-1}(\bar{\varepsilon})
$$


Then for every neighborhood $\mathcal{U} \ni F$ there exists an open set $\mathcal{V} \subset \mathcal{U}$ such that

$$
\mathcal{V} \cap \mathcal{U} \mathcal{K}_{k, n}(\bar{\varepsilon})=\emptyset
$$

Proof. The collections $\mathcal{U} \mathcal{K}_{k+1, n}$ and $\mathcal{U K}_{k, n-1}$ are closed, see Lemma 10.4. Let $\mathcal{U} \supset \mathcal{V}_{0} \ni F$ be a neighborhood with

$$
\mathcal{V}_{0} \cap\left(\mathcal{U K}_{k+1, n} \cup \mathcal{U} \mathcal{K}_{k, n-1}\right)=\emptyset \text {. }
$$

According to Theorem 10.9 there is $N \geq 1$ and a neighborhood $\mathcal{V}_{1} \subset \mathcal{V}_{0}$ such that for all $F \in \mathcal{V}_{1}$ all the tangencies between $W^{u}\left(\beta_{k}(\tilde{F})\right)$ and $W^{s}\left(\beta_{n}(\tilde{F})\right)$ in $K_{n}^{s}(\tilde{F})$ are in a bounded curve $C_{k, N}(\tilde{F})$. The maps in $\mathcal{H}_{\Omega}^{n}(\bar{\varepsilon})$ are analytic. This allows us to remove all finitely many tangencies and get an open set $\mathcal{V} \subset \mathcal{V}_{1}$ such that $\mathcal{V} \cap \mathcal{U} \mathcal{K}_{k, n}=\emptyset$.

For $F \in \mathcal{H}_{\Omega}^{n}(\bar{\varepsilon})$ define

$$
\Delta_{F}^{n}=\min \left\{n^{\prime}-k^{\prime} \mid F \in \mathcal{K}_{k^{\prime}, n^{\prime}}(\bar{\varepsilon}), k^{\prime}<n^{\prime} \leq n\right\} .
$$

Corollary 10.11. Let $F \in \mathcal{H}_{\Omega}^{n}(\bar{\varepsilon})$, with $\bar{\varepsilon}>0$ small enough, and $\Delta_{F}^{n}<\infty$. Then for every neighborhood $\mathcal{U} \ni F$ there exists an open set $\mathcal{V} \subset \mathcal{U}$ such that

$$
\Delta_{\tilde{F}}^{n}>\Delta_{F}^{n}
$$

for all $\tilde{F} \in \mathcal{V}$.

Proof. Let

$$
X=\left\{\left(k^{\prime}, n^{\prime}\right) \mid k^{\prime}<n^{\prime} \leq n \text { and } n^{\prime}-k^{\prime}<\Delta_{F}\right\}
$$

and

$$
Y=\left\{\left(k^{\prime}, n^{\prime}\right) \mid k^{\prime}<n^{\prime} \leq n, n^{\prime}-k^{\prime}=\Delta_{F}^{n}, \quad F \in \mathcal{K}_{k^{\prime}, n^{\prime}}(\bar{\varepsilon})\right\} .
$$

Observe,

$$
F \notin \bigcup_{\left(k^{\prime}, n^{\prime}\right) \in X} \mathcal{K}_{k^{\prime}, n^{\prime}}(\bar{\varepsilon})=\bigcup_{n^{\prime}-k^{\prime}=\Delta_{F}^{n}-1} \mathcal{U K}_{k^{\prime}, n^{\prime}}(\bar{\varepsilon})
$$

which is a finite union of closed set, see Lemma 10.4. Choose, $F \in$ $\mathcal{U}_{0} \subset \mathcal{U}$ such that

$$
\mathcal{U}_{0} \cap \bigcup_{\left(k^{\prime}, n^{\prime}\right) \in X} \mathcal{K}_{k^{\prime}, n^{\prime}}(\bar{\varepsilon})=\emptyset .
$$

Now apply repeatly Lemma 10.10 to erase the points $\left(k^{\prime}, n^{\prime}\right) \in Y$. We constructed $\mathcal{V} \subset \mathcal{U}_{0}$ such that for $\tilde{F} \in \mathcal{V}$

$$
\Delta_{\tilde{F}}>\Delta_{F}
$$


Proof of Theorem 10.1. Lemma 10.4 gives $\mathcal{U} \mathcal{K}_{0, n}(\bar{\varepsilon})$ is closed. In particular, the maps without heteroclinic tangencies form an open set in $\mathcal{I}_{\Omega}^{n}(\bar{\varepsilon})$. Let $\mathcal{U} \subset \mathcal{I}_{\Omega}^{n}(\bar{\varepsilon})$ and assume that every map in $\mathcal{U}$ has a tangency. We have

$$
\Delta_{F}^{n} \leq n,
$$

for $F \in \mathcal{U}$. There is an $F_{0} \in \mathcal{U}$ which has a tangency and where $\Delta_{F_{0}}^{n}$ is maximal. This contradicts Corollary 10.11 .

The following Lemmas are a preparation for the proof of Theorem 10.3

Lemma 10.12. For $\bar{\varepsilon}>0$ small enough, there exist $n>k \geq 1$ such that for $s \geq 1$ large enough

$$
\mathcal{I}_{\Omega}^{n+s}(\bar{\varepsilon}) \cap \mathcal{K}_{k, n}(\bar{\varepsilon}) \neq \emptyset .
$$

More precisely, there exists an $F \in \mathcal{I}_{\Omega}^{n+s}(\bar{\varepsilon}) \cap \mathcal{K}_{k, n}(\bar{\varepsilon})$ such that $\Gamma_{\infty}\left(F_{k-1}\right)$ is tangent to $M_{1}^{n-k+1}\left(F_{k-1}\right)$.

Proof. Let $\Omega=\Omega^{h} \times \Omega^{v}$ and $\mathcal{W}_{\Omega}^{n}(\bar{\varepsilon}) \subset \mathcal{H}_{\Omega}^{n}(\bar{\varepsilon})$ consists of the maps that have a periodic point of period $2^{n}$ with multiplier $-1, n \geq 1$. Choose a unimodal family $a \mapsto f_{a} \in \mathcal{U}_{\Omega^{h}}, a \in\left(-a_{0}, a_{0}\right)$, which intersects transversally $W^{s}\left(f_{*}\right)$ at $a=0$. For $a_{0}>0$ small enough we can consider the family

$$
F_{a, t}(x, y)=\left(f_{a}(x)-t \cdot \epsilon(x, y), x\right) \in \mathcal{H}_{\Omega}(\bar{\varepsilon}),
$$

with $a \in\left(-a_{0}, a_{0}\right)$ and $t \in[0,1]$. Let

$$
W^{n}=\left\{(a, t) \mid F_{a, t} \in \mathcal{W}_{\Omega}^{n}(\bar{\varepsilon})\right\}
$$

and

$$
W=\left\{(a, t) \mid F_{a, t} \in \mathcal{I}_{\Omega}(\bar{\varepsilon})\right\} .
$$

For $n \geq 1$ large enough and $\bar{\varepsilon}>0$ small enough $W$ and $W^{n}$ are graphs of analytic functions $t \mapsto a(t)$ and $t \mapsto a_{n}(t)$. Moreover, $a_{n} \rightarrow a$ (exponentially fast). Finally, let

$$
\begin{aligned}
I^{n} & =\left\{(a, t) \mid F_{a, t} \in \mathcal{I}_{\Omega}^{n}(\bar{\varepsilon})\right\} \\
& =\left\{(a, t) \mid a_{n-1}(t)<a<a_{n}(t)\right\} .
\end{aligned}
$$

These statements follows from the fact that the family $a \mapsto f_{a}$ intersects $W^{s}\left(f_{*}\right)$ transversally and the hyperbolicity of the Hénon-renormalization operator.

Choose $t, \tilde{t} \in[0,1]$. Consider the maps $F=F_{a(t), t} \in \mathcal{I}_{\Omega}(\bar{\varepsilon})$ with average Jacobian $b>0$ and $\tilde{F}=F_{a(\tilde{t}), \tilde{t}} \in \mathcal{I}_{\Omega}(\bar{\varepsilon})$ with average Jacobian

\footnotetext{
${ }^{1}$ See 97 for the definition of $\Gamma_{\infty}\left(F_{k-1}\right)$ and Figure 3.1 for $M_{1}^{n-k+1}\left(F_{k-1}\right)$.
} 
$\tilde{b}>b$. We will use a short hand notation for the invariants of $\oint 8$; let $\kappa_{n}=\kappa_{R^{n} F}$ and $\tilde{\kappa}_{n}=\kappa_{R^{n} \tilde{F}}$. For $n \geq 1$ large enough we have, as a consequence of Theorem 8.3 ,

$$
\kappa_{n}>2^{n} \cdot\left\{\frac{|\ln b|}{\left|\ln \sigma^{2}\right|}-1\right\}>2^{n} \cdot\left\{\frac{|\ln \tilde{b}|}{\left|\ln \sigma^{2}\right|}+1\right\}>\tilde{\kappa}_{n} .
$$

Hence, for $n \geq 1$ large enough we can find $k \geq 1$ such that

$$
\kappa_{n+1}>k-1>\tilde{\kappa}_{n+1} .
$$

The map $F$ has the property that

$$
M_{1}^{k-1}\left(R^{n+1} F\right) \cap D^{\tau}\left(R^{n+1} F\right)=\emptyset .
$$

In other words, see Figure 3.1, that

$$
M_{1}^{n+1+k-1}(F) \cap\left[p_{0}^{n+1}, p_{1}^{n+1}\right]^{u}=\emptyset .
$$

The map $\tilde{F}$ has the property that $M_{1}^{k-1}\left(R^{n+1} \tilde{F}\right)$ has a nonempty transversal intersection with the boundary of $D^{\tau}\left(R^{n+1} \tilde{F}\right)$. In particular,

$$
M_{1}^{n+1+k-1}(\tilde{F}) \cap\left[\tilde{p}_{0}^{n+1}, \tilde{p}_{1}^{n+1}\right]^{u} \neq \emptyset,
$$

and consists of transversal intersections. The transversal intersections given in (10.15) and (10.16) persist locally. Hence, for $s \geq 1$ large enough the set $I^{n+k+s}$ will contain maps with a transverse intersection of type

$$
M_{1}^{n+1+k-1} \cap\left[p_{0}^{n+1}, p_{1}^{n+1}\right]^{u} \neq \emptyset,
$$

and also maps without any intersection between $M_{1}^{n+1+k-1}$ and $\left[p_{0}^{n+1}, p_{1}^{n+1}\right]^{u}$. The connectivity of $I^{n+k+s}$, see (10.14), implies that there are maps in $I^{n+k+s}$ for which there is a tangency between $\left[p_{0}^{n+1}, p_{1}^{n+1}\right]^{u}$ and $M_{1}^{n+k}$. Recall,

$$
\left[p_{0}^{n+1}, p_{1}^{n+1}\right]^{u} \subset W^{u}\left(\beta_{n}\right)
$$

and

$$
M_{1}^{n+k} \subset W^{s}\left(\beta_{n+k}\right) .
$$

Hence,

$$
\Gamma_{\infty}\left(F_{n-1}\right) \pi M_{1}^{k+1}\left(F_{n-1}\right) .
$$

Moreover, for $s \geq 1$ large enough, $\mathcal{I}_{\Omega}^{n+k+s}(\bar{\varepsilon}) \cap \mathcal{K}_{n, n+k}(\bar{\varepsilon}) \neq \emptyset$.

The following definition is illustrated by Figure 10.3 .

Definition 10.1. Given a map $F \in \mathcal{H}_{\Omega}^{k}(\bar{\varepsilon})$, with $\bar{\varepsilon}>0$ small enough and $k \geq 1$. If $\Gamma_{\infty}(F) \cap M_{1}^{k}(F)=\emptyset$ then define

$$
j_{k}=j_{k}(F)=\max \left\{j \geq 2 \mid \Gamma_{j} \cap M_{1}^{k} \neq \emptyset\right\} .
$$


Otherwise, if $\Gamma_{\infty} \cap M_{1}^{k} \neq \emptyset$ let

$$
j_{k}=j_{k}(F)=\max \left\{j \geq 2 \mid \Gamma_{j} \cap M_{1}^{k}=\emptyset\right\} .
$$

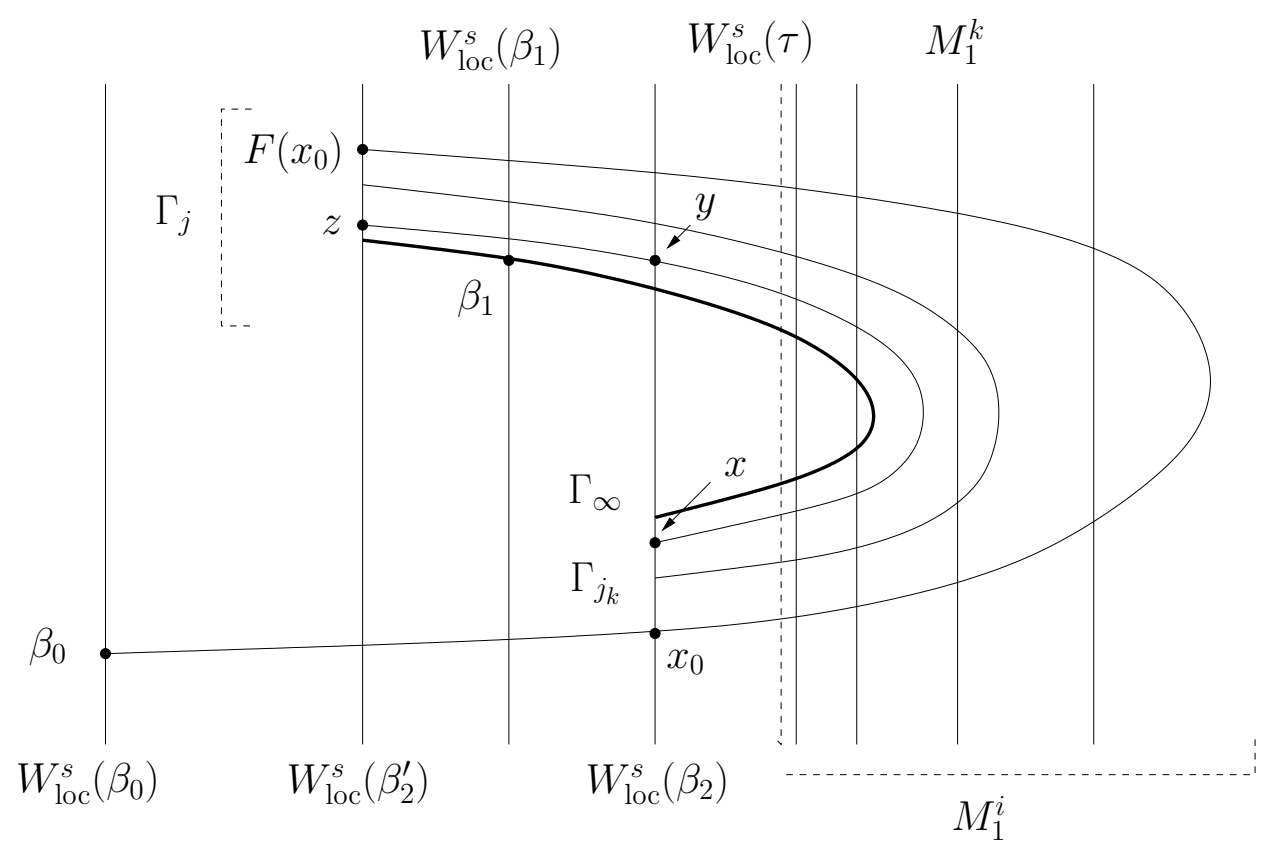

FiguRe 10.3.

Lemma 10.13. $j_{k}(F)$ is a topological invariant.

Proof. Let $x_{0} \in W^{u}\left(\beta_{0}\right)$ be the first intersection, coming from $\beta_{0}$ going along $W^{u}\left(\beta_{0}\right)$, with $W_{\text {loc }}^{s}\left(\beta_{2}\right)$, see Figure 10.3, Proposition 5.3 implies that this point is topologically defined. Notice, that

$$
\Gamma_{2}=\left[x_{0}, F\left(x_{0}\right)\right]^{u} \subset W^{u}\left(\beta_{0}\right) .
$$

Thus $\Gamma_{2}$ is topologically defined. Now, assume that

$$
\Gamma_{j}=[z, x]^{u} \subset W^{u}\left(\beta_{0}\right),
$$

is topologically defined, with $z \in W_{\mathrm{loc}}^{s}\left(\beta_{2}^{\prime}\right)$ and $x \in W_{\mathrm{loc}}^{s}\left(\beta_{2}\right)$. Then, when $\bar{\varepsilon}>0$ is small enough, there is a unique intersection point

$$
y \in(z, x)^{u} \cap W_{\text {loc }}^{s}\left(\beta_{2}\right) .
$$

This point is topologically defined. Now observe that

$$
\Gamma_{j+1}=F\left([z, y]^{u}\right) .
$$

We proved that all $\Gamma_{j}, j \geq 2$, are topologically defined. 
Recall from $\$ 8$ that $D^{\tau} \subset D_{1}$ is the connected component of $D_{1} \backslash$ $W_{\text {loc }}^{s}(\tau)$ which does not contain $\beta_{1}$, see Figure 8.1 and that $M_{1}^{k}$ is on the right of $W_{\mathrm{loc}}^{s}(\tau)$. Observe,

$$
M_{1}^{k} \cap D_{1}=M_{1}^{k} \cap D^{\tau} .
$$

According to Lemma 8.1, this intersection is a topologically defined set. Furthermore, notice that

$$
\Gamma_{j} \cap M_{1}^{k} \subset D_{1}
$$

Hence, the intersections

$$
\Gamma_{j} \cap M_{1}^{k}
$$

are topologically defined. We proved that $j_{k, n}$ is a topological invariant.

Fix a map $F \in \mathcal{I}_{\Omega}^{n+s}(\bar{\varepsilon})$, with $\bar{\varepsilon}>0$ small enough and $s \geq 1$. We will need the objects defined in $\$ 7$. In that section we used the graphs $\Gamma_{j}=\Gamma_{j}\left(F_{n}\right), j=2,3, \ldots$, and $\Gamma_{\infty}=\Gamma_{\infty}\left(F_{n}\right)$. Apply Corollary 7.2 to obtain a $\rho=\rho(F, n)<1$ such that for $j \geq 2$

$$
\left|\Gamma_{j}(y)-\Gamma_{\infty}(y)\right| \geq \rho^{j}
$$

holds on a neighborhood of $F$.

Proof of Theorem 10.3 Apply Lemma 10.12: for every $\bar{\varepsilon}>0$ small enough, there exist $k, n>0$ such that for every $s \geq 1$ large enough there is a map $F \in \mathcal{I}_{\Omega}^{n+k+s}(\bar{\varepsilon}) \cap \mathcal{K}_{n, n+k}(\bar{\varepsilon})$ such that $\Gamma_{\infty}\left(F_{n-1}\right)$ is tangent to $M_{1}^{k}\left(F_{n-1}\right)$. Use Theorem 10.1 to choose a sequence $G_{m} \in \mathcal{I}_{\Omega}^{n+k+s}(\bar{\varepsilon})$ of Morse-Smale maps converging to $F$. Now

$$
j_{k}\left(R^{n-1} G_{m}\right) \rightarrow \infty,
$$

for $m \rightarrow \infty$. Otherwise, suppose that for a subsequence $j_{k}\left(R^{n-1} G_{m}\right) \leq$ $j$ stays bounded. Let $\Gamma_{j}^{m}$ and $\Gamma_{\infty}^{m}$ be the corresponding graphs of the maps $R^{n-1} G_{m}$. Then

$$
\min _{y}\left|\Gamma_{j+1}^{m}(y)-\Gamma_{\infty}^{m}(y)\right| \rightarrow 0
$$

for $m \rightarrow \infty$. This contradicts inequality (10.17). We found countable many Morse-Smale maps of different type accumulating at $F$.

Remark 10.3. Notice that a map $F \in \mathcal{I}_{\Omega}^{n}(\bar{\varepsilon})$ might have heteroclinic tangencies in places other then the ones discussed in the proof of Theorem 10.3. The proof only describes some particular collection of boundary curves of Morse-Smale components but there might be many more components of other types. 
Remark 10.4. Observe that the results of this section also hold when we consider two dimensional analytic families in $\mathcal{H}_{\Omega}(\bar{\varepsilon})$ transverse to $\mathcal{I}_{\Omega}(\bar{\varepsilon})$.

\section{Appendix: VARIATION OF THE $\beta_{0}$-UnStable MANifOLD}

Let us consider a one-parameter real analytic family of Hénon-like maps

$$
F_{t}(x, y)=\left(f(x)-t \gamma(x, y)+O\left(t^{2}\right), x\right), \quad \text { where }\left.\gamma \equiv \frac{d F_{t}}{d t}\right|_{t=0}
$$

is the parameter velocity of this family at $f$.

Let $\beta_{t}=\beta_{0}(t)$ be the saddle fixed point of $F_{t}$ with positive eigenvalues, and let $\lambda_{t}>1$ be its repelling eigenvalue. Below we will calculate the first variation of the unstable manifold $W^{u}\left(\beta_{t}\right)$ at $t=0$. To this end let us linearize $F_{t} \mid W^{u}\left(\beta_{t}\right)$ :

$$
\Phi_{t}: \mathbb{R} \rightarrow W^{u}\left(\beta_{t}\right), \quad \Phi_{t}\left(\lambda_{t} s\right)=F_{t}\left(\Phi_{t}(s)\right) .
$$

Note that for $t=0, \Phi_{0}(s)=\left(\phi_{0}(s), \phi_{0}\left(s / \lambda_{0}\right)\right)$, where $\phi_{0}: \mathbb{R} \rightarrow \mathbb{R}$ is the linearizing map of $f$ at the fixed point $\beta_{0}$.

Let $\phi_{t}$ be the first coordinate of $\Phi_{t}$. Then

$$
\phi_{t}\left(\lambda_{t} s\right)=f\left(\phi_{t}(s)\right)-t \gamma\left(\phi_{t}(s), \phi_{t}\left(s / \lambda_{t}\right)\right)+O\left(t^{2}\right) .
$$

Let

$$
\lambda_{t}=\lambda_{0}+\mu t+O\left(t^{2}\right), \quad \phi_{t}(s)=\phi_{0}(s)+\psi(s) t+O\left(t^{2}\right) .
$$

Plugging it into (10.19) and keeping only linear terms in $t$, we obtain the following equation:

$$
\phi_{0}^{\prime}\left(\lambda_{0} s\right) \mu s+\psi\left(\lambda_{0} s\right)=f^{\prime}\left(\phi_{0}(s)\right) \psi(s)-\gamma\left(\phi_{0}(s), \phi_{0}\left(s / \lambda_{0}\right)\right) .
$$

Let us now look what happens when $\phi_{0}\left(s_{c}\right)=c$. 2 Letting $s_{v}=\lambda_{0} s_{c}$, we have $\phi_{0}\left(s_{v}\right)=v$. Since $v$ is the maximum of $\phi_{0}$, the first terms in the both sides of the above equation vanish, and we obtain:

$$
\psi\left(s_{v}\right)=-\gamma\left(c, c_{-1}\right),
$$

where $c_{-1}=\phi_{0}\left(\tau_{c} / \lambda_{0}\right) \in f^{-1}(c)$ is a precritical point (there are infinitely many values of $s_{c}$; they split into two classes corresponding to the upper and lower critical point on the parabola, which correspond to the two precritical points $c_{-1}$ ). It allows us to estimate the distance from the turning points of the unstable manifold to the critical value. A turning point of the curve $W^{u}\left(\beta_{t}\right)$ is a point with vertical tangency.

\footnotetext{
${ }^{2}$ recall that $c$ is the critical point and $v$ is the critical value of $f$.
} 
Lemma 10.14. In the above one-parameter family $F_{t}$ of Hénon-like maps, the horizontal distance from the first and the third turns of the unstable manifold $W^{u}\left(\beta_{t}\right)$ to the turning point $(v, c)$ of the parabola $x=$ $f(y)$ is $-\gamma\left(c, c_{-1}\right) t+O\left(t^{2}\right)$, where we should take the lower precritical point $c_{-1}$ for the first turning point, and the upper precritical point for the second one.

Proof. According to (10.21), the horizontal distance from $\phi_{t}\left(s_{v}\right)$ to $v=$ $\phi_{0}\left(s_{v}\right)$ is $-\gamma\left(c, c_{-1}\right) t+O\left(t^{2}\right)$. However, $\Phi_{t}\left(s_{v}\right)$ is not the turning point $\left(x_{t}, y_{t}\right)$ of the unstable manifold $W^{u}\left(\beta_{t}\right)$, so we need to show that $\mid x_{t}-$ $\phi_{t}\left(s_{v}\right) \mid=O\left(t^{2}\right)$.

Let $x_{t}=\phi_{t}\left(s_{t}\right)$. Let us estimate $\left|s_{t}-s_{v}\right|$. Since $\phi_{t}^{\prime}\left(s_{t}\right)=0$, the second equation of (10.20) yield:

$$
0=\phi_{0}^{\prime}\left(s_{t}\right)+\psi^{\prime}\left(s_{t}\right) t+\ldots
$$

Linearizing this equation in $s$ near $s_{v}$, using that $\phi_{0}^{\prime}\left(s_{v}\right)=0$, we obtain:

$$
\phi_{0}^{\prime \prime}\left(s_{v}\right)\left(s_{t}-s_{v}\right)+\left(\psi^{\prime}\left(s_{v}\right)+\psi^{\prime \prime}\left(s_{v}\right)\left(s_{t}-s_{v}\right)\right) t+O\left(t^{2}\right)=0 .
$$

Hence,

$$
s_{t}-s_{v}=\frac{\psi^{\prime}\left(s_{v}\right)}{\phi^{\prime \prime}\left(s_{v}\right)} \cdot t+O\left(t^{2}\right),
$$

provided $\phi_{0}^{\prime \prime}\left(s_{v}\right) \neq 0$. But the latter is actually true, which is easily checked by differentiating twice at $s_{c}$ the linearization equation $\phi_{0}\left(\lambda_{0} s\right)=f_{0}\left(\phi_{0}(s)\right)$ (using that the critical point $c$ of $f_{0}$ is non-degenerate).

Finally, we conclude:

$$
\begin{aligned}
x_{t} & =\phi_{t}\left(s_{t}\right) \\
& =\phi_{0}\left(s_{t}\right)+\psi\left(s_{t}\right) t+O\left(t^{2}\right) \\
& =\phi_{0}\left(s_{v}\right)+\phi_{0}^{\prime}\left(s_{v}\right) \cdot a t+\psi\left(s_{v}\right) t+O\left(t^{2}\right) \\
& =v+\psi\left(s_{v}\right) t+O(t 2)=\phi_{t}\left(s_{v}\right)+O\left(t^{2}\right) .
\end{aligned}
$$

Note that it is reasonable to assume that $\gamma\left(c, c_{-1}\right)$ is positive at the upper critical point and negative at the lower one, and has the absolute value of order 1 . Then the first turning point of $W^{u}\left(\beta_{t}\right)$ is on the right from $(v, c)$ (for $t>0$ ), while the third one is on the left (as we always draw), and as $t \rightarrow 0$, they move toward $(v, c)$ with a speed of order 1 .

Let $w(F)$ be the horizontal distance between the first and the third turning points of the unstable manifold $W^{u}(\beta)$ (which measures the width of the horseshoe near the tip). Let $c^{+}$and $c^{-}$denote the upper and the lower precritical points of $f$ respectively. 
Lemma 10.15. For the family (10.18),

$$
\left.\frac{d w\left(F_{t}\right)}{d t}\right|_{t=0}=\left.\int_{c^{-}}^{c^{+}} \frac{d \mathrm{Jac} F_{t}(c, y)}{d t}\right|_{t=0} d y
$$

Proof. According to Lemma 10.14,

$$
\begin{aligned}
\left.\frac{d w\left(F_{t}\right)}{d t}\right|_{t=0} & =\gamma\left(c, c^{+}\right)-\gamma\left(c, c^{-}\right)=\int_{c^{-}}^{c^{+}} \frac{\partial \gamma(c, y)}{\partial y} d y \\
& =\left.\int_{c^{-}}^{c^{+}} \frac{d \mathrm{Jac} F_{t}(c, y)}{d t}\right|_{t=0} d y
\end{aligned}
$$

The last formula can also be written in the following form: For a Hénon-like map $F=(f-\varepsilon, x) \in \mathcal{H}_{\Omega}(\bar{\varepsilon})$,

$$
\delta w(f)=\int_{c^{-}}^{c^{+}} \delta \operatorname{Jac} F(c, y) d y
$$

Lemma 10.16. For $F(x, y)=(f(x)-b a(x, y), x) \in \mathcal{H}_{\Omega}(\bar{\varepsilon})$, assume

$$
C^{-1} \leq|\partial a / \partial y| \leq C \text {. }
$$

Then $w(F) \asymp b$ for $b \leq b_{0}$, with the constants depending only on $\Omega, \bar{\varepsilon}$ and $C$.

Proof. Consider $b \in\left[0, b_{0}\right]$ as a small parameter. Then by the variational formula (10.22)

$$
\left.\frac{d w\left(F_{b}\right)}{d b}\right|_{b=0}=\int_{c^{-}}^{c^{+}} \frac{\partial a}{\partial y}(c, y) d y \asymp 1 .
$$

Moreover, the second derivative $d 2 w\left(F_{b}\right) / d b 2$ is bounded on the interval $\left[0, b_{0}\right]$, uniformly over $F \in \mathcal{H}_{\Omega}(\bar{\varepsilon})$ (since $w(F)$ is a $C 2$-smooth, in fact analytic, functional on this space), and the conclusion follows by elementary calculus.

The asymptotic expression for $R^{n} F$ as given in (2.3) implies:

Proposition 10.17. For $F_{b} \in \mathcal{I}_{\Omega}(\bar{\varepsilon})$, be a family of infinitely renormalizable maps parametrized by the average Jacobian $b=b_{F_{b}}$. Then

$$
\lim _{b \rightarrow 0} \frac{w\left(R^{n} F_{b}\right)}{b^{2^{n}}}=a(c) \cdot\left(c^{+}-c^{-}\right)
$$

where $b$ is the average Jacobian of $F$ and $c^{ \pm}$are the preimages of the critical point $c$ of $f_{*}$. And $x \mapsto a(x)$ the universal function given in (2.3). 


\section{Appendix: Open Problems}

Let us finish with some questions related to the previous discussion.

Problem I:

The following questions are inspired by the results of $\S 9$ on stable laminations.

(1) A wandering domain is an open set in the basin of attraction of $\mathcal{O}_{F}$. Do wandering domains exist?

(2) If a map $F \in \mathcal{I}_{\Omega}(\bar{\varepsilon})$ does not have wandering domains then the union $\mathcal{F}^{s}$ of all stable manifolds of periodic points are dense in the domain of $F$. Does there exist $F \in \mathcal{I}_{\Omega}(\bar{\varepsilon})$ such that $\mathcal{F}^{s}$ is not laminar even if there are no heteroclinic tangencies?

(3) For $F \in \mathcal{I}_{\Omega}(\bar{\varepsilon})$ let $\mathcal{F}_{\tau}^{s}$ be the union of stable manifolds of the points in the orbit of the tip. Is $\mathcal{F}_{\tau}^{s}$ dense in $\operatorname{Dom}(F)$ ?

\section{Problem II:}

It is shown in CLM that the unique invariant measure on the Cantor attractor $\mathcal{O}_{F}$ has characteristic exponents 0 and $\ln b_{F}<0$. Can the stable characteristic exponent of the tip $\tau_{F}$ differ from $\ln b_{F}$ (compare Proposition 3.8)?

\section{Problem III:}

Can we still speak of rigidity of the Cantor attractor $\mathcal{O}_{F}$ ?

(1) Are the Cantor attractors rigid within the topological conjugacy classes?

(2) Prove or disprove that two Cantor attractors $\mathcal{O}_{F}$ and $\mathcal{O}_{\tilde{F}}$ are smoothly equivalent if and only if they have the same average Jacobian.

\section{Problem IV:}

(1) Can different Morse-Smale components

$$
M S_{1}, M S_{2} \subset \bigcup_{n \geq 0} \mathcal{I}_{\Omega}^{n}(\bar{\varepsilon})
$$

have the same type, that is the maps in $M S_{1}$ are conjugate to the maps in $M S_{2}$ ?

(2) As we have shown, the Morse-Smale Hénon maps are dense in the zero entropy region with small Jacobian. Are they dense in the full zero entropy region of dissipative Hénon maps? How about other real analytic families of dissipative two dimensional maps? 
(3) The discussion that led to Theorem 10.3 was based on the renormalization structure. However, the non-locally finiteness of the collection of Morse-Smale components might be a more general phenomenon. Study the combinatorics of Morse-Smale components in other real analytic families of dissipative two dimensional maps.

(4) Are the real Morse-Smale Hénon maps from Theorem 10.3 hyperbolic on $\mathbb{C}^{2}$ ? To what extent determines the topology of the real heteroclinic web the topology of the corresponding Hénon map on $\mathbb{C}^{2}$ ?

\section{NOMENCLATURE}

$\mathcal{A}_{F}$ global attractor $\$ 4$

$\mathcal{A}_{F}^{n} n^{\text {th }}$-scale attractor $\$ 4$

$b_{F}$ average Jacobian, §2

$B_{0}$ non-escaping points, 4

$B_{v^{n}}$ renormalization piece around the tip of level $n$, §2

$\hat{\beta}_{n}$ fixed point of $R^{n} F, 93$

$\beta_{n}$ periodic point, $\$ 3$

$\beta_{n}^{\prime}$ periodic point, $\$ 3$

$\mathcal{C}_{F}$ non-laminar points in $\mathcal{A}_{F}, 86$

$\gamma_{\infty}$ curve, Figure 7.1

$\gamma_{j}$ curve, Figure 7.1

$\Gamma_{\infty}$ curve, Figure 7.1

$\Gamma_{j}$ curve, Figure 7.1

$D_{n}$ periodic domain containing the tip, 93

$D^{\tau}$ domain bounded by $W_{\mathrm{loc}}^{s}(\tau)$ and $W^{u}\left(\beta_{0}\right)$, 88

$E_{k, n}$ heteroclinic points, $\$ 4$

$E_{k, n}^{s}$ heteroclinic points, $₫ 9$

$\Phi_{0}^{n}$ coordinate change, $\$ 3$

$\Phi_{k}^{k+1}$ coordinate change, $₫ 3$

$\mathcal{H}_{\Omega}^{n}(\bar{\varepsilon}) n$-times renormalizable maps, $\S 2$

$\mathcal{I}_{\Omega}(\bar{\varepsilon})$ infinitely renormalizable maps, \$2

$\mathcal{I}_{\Omega}^{n}(\bar{\epsilon}) n$-times renormalizable maps with a periodic attractor of period $2^{n}, \S 2$

$K_{n}^{u}$ fundamental domain in $W^{u}\left(\beta_{n}\right)$, §4

$K_{n}^{s}$ fundamental domain in $W^{s}\left(\beta_{n}\right)$, §9

$\mathcal{K}_{k, n}(\bar{\varepsilon})$ maps with heteroclinic tangencies, Definition 4.3

$\mathcal{U K}_{k, n}(\bar{\varepsilon})$ maps in $\mathcal{K}_{k^{\prime}, n^{\prime}}(\bar{\varepsilon})$ with $k \leq k^{\prime}<n^{\prime} \leq n$, Definition 4.3

$\boldsymbol{\kappa}_{F}$ topological invariant, 88

$\lambda_{n}$ stable eigenvalue of $\beta_{n}, \sqrt{6}$ 
$\mu_{n}$ unstable eigenvalue of $\beta_{n}$, §6

$\hat{M}_{i}^{n}$ component stable manifold of $\hat{\beta}_{n}$, 乌3

$M_{i}^{n}$ component stable manifold of $\beta_{n}, 93$

$\mathcal{O}_{F}$ critical Cantor set, §2

$\hat{p}_{i}^{n}$ heteroclinic point of $R^{n} F, \S 3$

$p_{i}^{n}$ heteroclinic point of $F, 93$, also Figure 3.1

$q_{i}$ heteroclinic point of $R^{n} F, \S 4$, also Figure 4.1

$q_{i}^{\prime}$ heteroclinic point of $R^{n} F, \& 4$, also Figure 4.1

$\sigma$ scaling factor of the unimodal fixed point, \$2

$\operatorname{Trap}_{n} n^{\text {th }}$-trapping region, $\$ 4$

$\tau_{F}$ tip, \$2

\section{REFERENCES}

[AM] J. M. Aarts, M. Martens. Flows on one-dimensional spaces, Fund. Math. 131 (1988).

[CEK] P. Collet, J. P. Eckmann, H. Koch. Period doubling bifurcations for families of maps on $\mathbb{R}^{n}$. J. Stat. Physics 25 (1980), 1-15.

[CLM] A. de Carvalho, M. Lyubich, M. Martens. Renormalization in the Hénon family, I: universality but non-rigidity, J. Stat. Phys. 121 No. 5/6, (2005), 611-669.

[C] S. Crovisier. Birth of homoclinic intersections: a model for the central dynamics of partially hyperbolic systems. arXiv: math.DS/0605387.

[dMP] W. de Melo, J. Palis. Geometric Theory of Dynamical Systems, Springer 1982.

[FMP] E. de Faria, W. de Melo \& A. Pinto. Global hyperbolicity of renormalization for $C^{r}$ unimodal mappings, Ann. of Math. 164 No. 3, (2006), 731-824.

[GST] J.-M. Gambaudo, S. van Strien \& C. Tresser. Hénon-like maps with strange attractors: there exist $C^{\infty}$ Kupka-Smale diffeomorphisms on $S^{2}$ with neither sinks nor sources. Nonlinearity 2 (1989), 287-304.

[H] P. Hartman. On local homeomorphisms of Euclidean spaces, Bol. Soc. Mexicana 5 (1960), 220-241.

[Mi1] J.W. Milnor. On the concept of attractor, Comm. Math. Phys. 99 (1985), 177-195.

[Mi2] J.W. Milnor. Attractor, Scholarpedia.

[P] J. Palis. On Morse-Smale dynamical systems, Topology 8 (1968), 385-404.

[PS] E. R. Pujals \& M. Sambarino. Homoclinic tangencies and hyperbolicity for surface diffeomorphisms. Ann. Math. 51 (2000), 961-1023.

[PSW] C. Pugh, M. Shub,\& A. Wilkinson. Hölder Foliations, Duke Math. J. 86 (1997), 517-546. Correction Vol. 105 (2000) 105-106.

Stony Brook University and University of Toronto 